DESY 10-218

December 2010

\title{
Quantum Leptogenesis I
}

\author{
A. Anisimov, ${ }^{1}$ W. Buchmüller, ${ }^{2}$ M. Drewes,${ }^{3}$ S. Mendizabal ${ }^{4}$ \\ ${ }^{1}$ Fakultät für Physik, Universität Bielefeld, 33615 Bielefeld, Germany \\ 2 Deutsches Elektronen-Synchrotron DESY, 22603 Hamburg, Germany \\ ${ }^{3}$ Institute de Théorie des Phénomènes Physiques EPFL, 1015 Lausanne, Switzerland \\ ${ }^{4}$ Institut für Theoretische Physik, Goethe-Universität, 60438 Frankfurt am Main, Germany
}

\begin{abstract}
Thermal leptogenesis explains the observed matter-antimatter asymmetry of the universe in terms of neutrino masses, consistent with neutrino oscillation experiments. We present a full quantum mechanical calculation of the generated lepton asymmetry based on Kadanoff-Baym equations. Origin of the asymmetry is the departure from equilibrium of the statistical propagator of the heavy Majorana neutrino, together with CP violating couplings. The lepton asymmetry is calculated directly in terms of Green's functions without referring to "number densities". Compared to Boltzmann and quantum Boltzmann equations, the crucial difference are memory effects, rapid oscillations much faster than the heavy neutrino equilibration time. These oscillations strongly suppress the generated lepton asymmetry, unless the standard model gauge interactions, which cause thermal damping, are properly taken into account. We find that these damping effects essentially compensate the enhancement due to quantum statistical factors, so that finally the conventional Boltzmann equations again provide rather accurate predictions for the lepton asymmetry.
\end{abstract}




\section{Introduction}

Standard thermal leptogenesis [1] provides a simple and elegant explanation of the origin of matter in the universe. Baryogenesis via leptogenesis naturally emerges in grand unified extensions of the Standard Model, which incorporate right-handed neutrinos and the seesaw mechanism, and the predicted connection between the cosmological matter-antimatter asymmetry and neutrino properties is in remarkable agreement with the present evidence for neutrino masses [2].

Leptogenesis is an out-of-equilibrium process in the high-temperature symmetric phase of the Standard Model. It makes use of nonperturbative properties of the Standard Model, the sphaleron processes which change baryon and lepton number [3], and it requires CP violation in the lepton sector and quantum interference in the thermal bath. Almost all quantitative studies of leptogenesis to date are based on Boltzmann's classical kinetic equations for the description of the nonequilibrium process [2].

In this article, we discuss a full quantum mechanical calculation of the generated lepton asymmetry based on Kadanoff-Baym equations [4] and the Schwinger-Keldysh formalism [5 7]. The main result has previously been reported in [8]. Here we give a detailed derivation of the result, discuss its interpretation and set the stage for future computations. Further work is still needed to obtain a 'quantum theory of leptogenesis' that can predict the cosmological matter-antimatter asymmetry in terms of neutrino properties without uncontrolable assumptions.

Conventional leptogenesis calculations based on kinetic equations suffer from a basic conceptual problem: the Boltzmann equations are classical equations for the time evolution of phase space distribution functions; the involved collision terms, however, are obtained from zero-temperature S-matrix elements which involve quantum interferences. This is in contrast to other successful applications of the Boltzmann equations in cosmology, like primordial nucleosynthesis, decoupling of photons or freeze-out of weakly interacting dark matter particles, where the collision terms arise from tree-level S-matrix elements. In the case of leptogenesis, clearly a full quantum mechanical treatment is necessary to understand the range of validity of the Boltzmann equations and to determine the size of possible corrections [9].

In recent years, various attempts have been made to go beyond Boltzmann equations. In [9], a solution of Kadanoff-Baym equations for leptogenesis has been found to leading order in a derivative expansion in terms of distribution functions satisfying the Boltzmann equations. Various thermal corrections, in particular quantum statistical factors and thermal masses, have been included [10-13]. Quantum Boltzmann equations have been derived from Kadanoff-Baym equations for scalar and Yukawa theories [14, 15] and for leptogenesis [16 19]. Except for [16], they do not contain memory effects, but they yield the correct statistical factors which go beyond the Boltzmann equations [8, 16, 17, 19, 20]. Quantum Boltzmann equations have important applications for resonant leptogenesis [16], flavoured leptogenesis [21,22] and $N_{2}$-leptogenesis [23]. Similar techniques have been developed for 


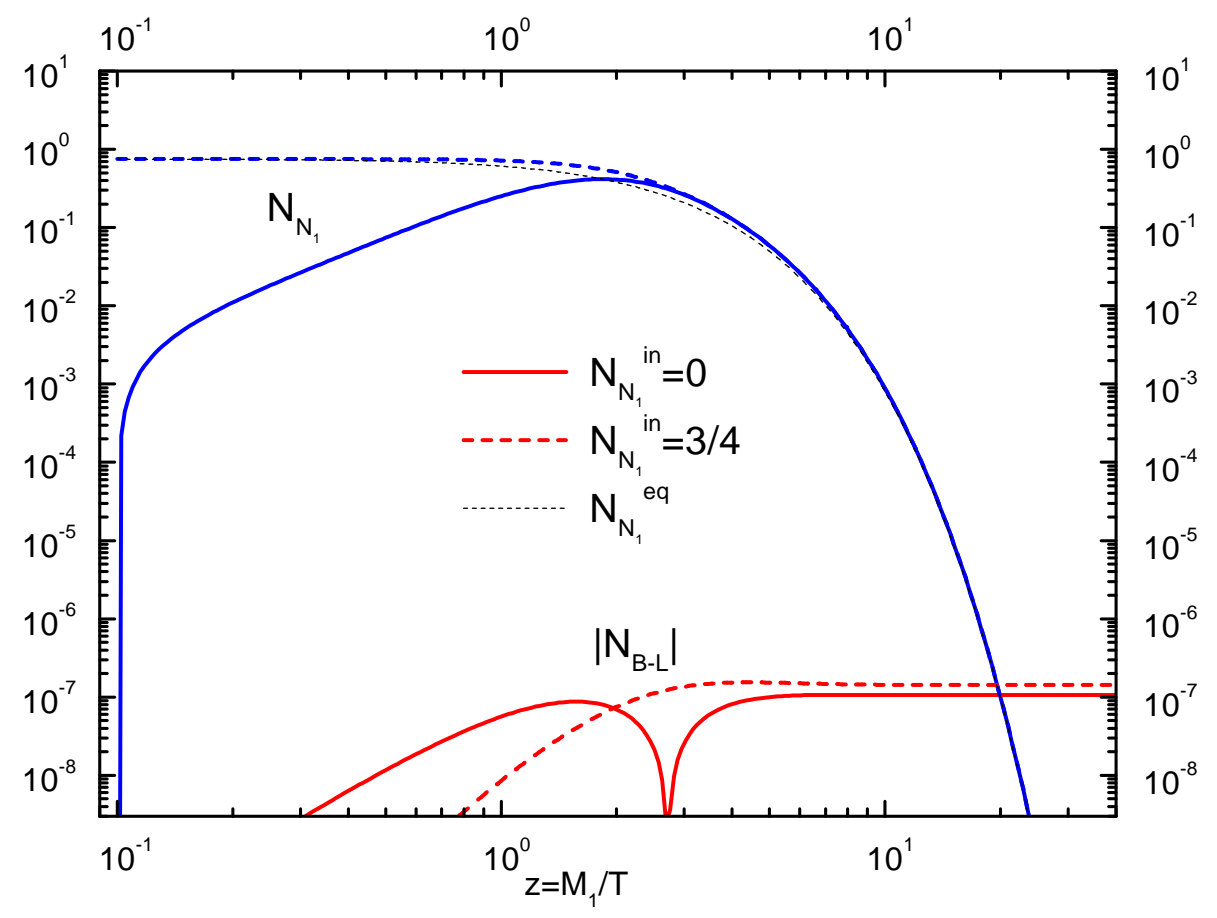

Figure 1: Evolution of heavy neutrino abundance $\mathrm{N}_{\mathrm{N}_{1}}$ and lepton asymmetry $\mathrm{N}_{\mathrm{B}-\mathrm{L}}$ for typical leptogenesis parameter: $M_{1}=10^{10} \mathrm{GeV}, \widetilde{m}_{1}=8 \pi \Gamma_{1}\left(v_{\mathrm{ew}} / M_{1}\right)^{2}=10^{-3} \mathrm{eV}, \epsilon=$ $10^{-6}$; the inverse temperature $z=M_{1} / T$ is the time variable. The dashed (full) lines correspond to thermal (vacuum) initial conditions for the heavy neutrino abundance; the dotted line represents the equilibrium abundance. From [30].

electroweak baryogenesis [24-27] and for coherent baryogenesis [28].

The quantum treatment of leptogenesis discussed in this paper is entirely based on Green's functions, thus avoiding all approximations needed to arrive at Boltzmann equations. Our work is based on [29], where the approach to thermal equilibrium has been discussed in terms of Green's functions for a toy model, a scalar field coupled to a large thermal bath. In leptogenesis it is the heavy neutrino which is weakly coupled to the standard model plasma containing many degrees of freedom. The nonequilibrium propagator of the heavy neutrino is obtained by solving the Kadanoff-Baym equations. The induced quantum corrections of the lepton (and Higgs) propagators then yield the wanted lepton asymmetry.

In general baryogenesis requires departure from thermal equilibrium. For the cosmological baryon asymmetry, this is provided by the Hubble expansion of the universe and, possibly, also by initial conditions. This can be seen in Fig. 1 where the time evolution of heavy neutrino abundance and lepton asymmetry, as predicted by the Boltzmann equations, are shown for two different initial conditions: thermal and zero heavy neutrino abundance. In the first case, the Hubble expansion leads to an excess of the neutrino abundance 
at $T \simeq 0.3 M_{1}$; shortly afterwards, washout processes are no longer in equilibrium and the lepton asymmetry is 'frozen in'. This is the standard out-of-equilibrium decay scenario of baryogenesis. In the second case, interactions with the thermal bath first bring the heavy neutrino into thermal equilibrium; due to the departure from thermal equilibrium during this time, an initial lepton asymmetry is generated. Around $T \simeq 0.3 M_{1}$, this asymmetry is washed out and, as in the first case, the final lepton asymmetry is generated. Remarkably, the initial and the final asymmetry have about the same size. For the generation of the initial asymmetry the change of temperature due to the Hubble expansion is not important. This allows us to make a significant technical simplification in our analysis. Since our goal is the comparison of Boltzmann and Kadanoff-Baym equations, we concentrate on the computation of the initial asymmetry at constant temperature. We expect differences between the classical and the quantum approach to be of similar size in the generation of the final asymmetry. In our numerical analysis we shall consider temperatures $T \lesssim M$, where the heavy neutrino production rate is not strongly affected by the effect of thermal masses of lepton and Higgs fields [11-13].

We consider an extension of the Standard Model with additional gauge singlet fermions, i.e., right-handed neutrinos, whose masses and couplings are described by the Lagrangian (sum over $i, j$ ),

$$
\mathcal{L}=\mathcal{L}_{S M}+\overline{\nu_{R i}} i \not \partial \nu_{R i}+\overline{l_{L i}} \tilde{\phi} \lambda_{i j}^{*} \nu_{R j}+\overline{\nu_{R j}} \lambda_{i j} l_{L i} \phi-\frac{1}{2} M_{i j}\left(\overline{\nu_{R i}^{c}} \nu_{R j}+\overline{\nu_{R}} \nu_{R i}^{c}\right)
$$

Here $\nu_{R}^{c}=C \bar{\nu}_{R}^{T}, C$ is the charge conjugation matrix and $\widetilde{\phi}=i \sigma_{2} \phi^{*} ; \mathrm{SU}(2)$ isospin indices have been omitted. For simplicity, we consider the case of hierarchical Majorana masses, $M_{k>1} \gg M_{1} \equiv M$, and small Yukawa couplings of the lightest heavy neutrino $N_{1} \equiv N$, $\lambda_{i 1} \ll 1$, such that the decay width is much smaller than the mass. Leptogenesis is then dominated by decays and inverse decays of $N$, and it is convenient to integrate out the heavier neutrinos. From Eq. (1.1) one then obtains the effective Lagrangian

$$
\begin{aligned}
\mathcal{L}= & \mathcal{L}_{S M}+\frac{1}{2} \bar{N} i \not \partial N+\overline{l_{L i}} \widetilde{\phi} \lambda_{i 1}^{*} N+N^{T} \lambda_{i 1} C l_{L i} \phi-\frac{1}{2} M N^{T} C N \\
& +\frac{1}{2} \eta_{i j} l_{L i}^{T} \phi C l_{L j} \phi+\frac{1}{2} \eta_{i j}^{*} \bar{l}_{L i} \widetilde{\phi} C{\overline{l_{L j}}}^{T} \widetilde{\phi},
\end{aligned}
$$

with $N=\nu_{R 1}+\nu_{R 1}^{c}$, and the familiar dimension-5 coupling

$$
\eta_{i j}=\sum_{k>1} \lambda_{i k} \frac{1}{M_{k}} \lambda_{k j}^{T} .
$$

Using this effective Lagrangian has the advantage that vertex- and self-energy contributions to the CP asymmetry in the heavy neutrino decay [31 33] are obtained from a single graph [9].

The paper is organized as follows. In Section 2 we present solutions of the Boltzmann equations for the heavy neutrino distribution function and the lepton asymmetry, which 
are useful for later comparison with the Kadanoff-Baym equations. Some results from nonequilibrium quantum field theory (QFT), in particular equilibrium correlation functions and Kadanoff-Baym equations, are recalled in Section 3. Section 4 contains some of the main results of this paper: analytic solutions of spectral function and statistical propagator for the heavy neutrino. These are needed for the computation of the lepton asymmetry, which is carried out in Section 5. A detailed comparison of the Boltzmann result and the Kadanoff-Baym result is given in Section 6, and numerical results for the generated lepton asymmetries are compared in Section 7. Summary and conclusions are given in Section 8, and various details, including equilibrium correlation functions, Feynman rules, a discussion of the zero-width limit and the computation of some integrals are contained in Appendices A - D.

\section{Boltzmann equations}

The Boltzmann equations for the time evolution of the distribution functions of heavy neutrinos, lepton and Higgs doublets are well known [34]. As discussed in the previous section, we focus on the generation of the 'initial asymmetry' (cf. Fig. 1), which allows us to neglect Hubble expansion and washout terms and to work at constant temperature $T$. The distribution function of the heavy neutrinos is then determined by the first-order differential equation 1

$$
\begin{aligned}
\frac{\partial}{\partial t} f_{N}\left(t, \omega_{\mathbf{p}}\right)= & -\frac{2}{\omega_{\mathbf{p}}} \int_{\mathbf{k}, \mathbf{q}}(2 \pi)^{4} \delta^{4}(k+q-p)\left(\lambda^{\dagger} \lambda\right)_{11} p \cdot k \\
& \times\left[f_{N}\left(t, \omega_{\mathbf{p}}\right)\left(1-f_{l}(k)\right)\left(1+f_{\phi}(q)\right)-f_{l}(k) f_{\phi}(q)\left(1-f_{N}\left(t, \omega_{\mathbf{p}}\right)\right)\right],
\end{aligned}
$$

with vacuum initial condition,

$$
f_{N}\left(0, \omega_{\mathbf{p}}\right)=0
$$

here $\omega_{\mathbf{p}}=\sqrt{M^{2}+\mathbf{p}^{2}}, k$ and $q$ are the energies of $N, l$ and $\phi$ with equilibrium distribution functions $f_{l}$ and $f_{\phi}$, respectively; the averaged decay matrix element is $\mid M(N(p) \rightarrow$ $l(k) \phi(q))\left.\right|^{2}=2\left(\lambda^{\dagger} \lambda\right)_{11} p \cdot k$ (cf. [9]). For the momentum integrations we use the notation

$$
\int_{\mathbf{p}} \ldots=\int \frac{d^{3} p}{(2 \pi)^{3} 2 \omega} \cdots
$$

In most leptogenesis calculations one directly computes the number density,

$$
n_{N}(t)=\int \frac{d^{3} p}{(2 \pi)^{3}} f_{N}\left(t, \omega_{\mathbf{p}}\right),
$$

\footnotetext{
${ }^{1}$ To simplify notation, we use the same symbol for the modulus of 3-momentum and 4-momentum, e.g., $k=|\mathbf{k}|$ and $k=(|\mathbf{k}|, \mathbf{k})$.
} 
assuming kinetic equilibrium.

The sum of decay and inverse decay widths, whose inverse is the time needed to reach thermal equilibrium [35], is given by

$$
\Gamma_{\mathbf{p}}=\left(\lambda^{\dagger} \lambda\right)_{11} \frac{2}{\omega_{\mathbf{p}}} \int_{\mathbf{k}, \mathbf{q}}(2 \pi)^{4} \delta^{4}(k+q-p) p \cdot k f_{l \phi}(k, q)
$$

where we have introduced the statistical factor (cf. [35])

$$
\begin{aligned}
f_{l \phi}(k, q) & =f_{l}(k) f_{\phi}(q)+\left(1-f_{l}(k)\right)\left(1+f_{\phi}(q)\right) \\
& =1-f_{l}(k)+f_{\phi}(q) .
\end{aligned}
$$

Neglecting the momentum dependence of the heavy neutrino width $\left(\Gamma_{\mathbf{p}} \equiv \Gamma\right)$, one easily obtains the solution of the Boltzmann equation (2.1) with vacuum initial condition,

$$
f_{N}\left(t, \omega_{\mathbf{p}}\right)=f_{N}^{e q}\left(\omega_{\mathbf{p}}\right)\left(1-e^{-\Gamma t}\right)
$$

where the equilibrium distribution is

$$
f_{N}^{e q}\left(\omega_{\mathbf{p}}\right)=\frac{1}{e^{\beta \omega_{\mathbf{p}}}+1}
$$

and $\beta=1 / T$ is the inverse temperature.

To compute the lepton asymmetry, we need the Boltzmann equation for the lepton distribution function,

$$
\begin{aligned}
\frac{\partial}{\partial t} f_{l}(t, k)=-\frac{1}{2 k} \int_{\mathbf{q}, \mathbf{p}}(2 \pi)^{4} \delta^{4}(k+q-p) \\
\quad \times\left[|M(l \phi \rightarrow N)|^{2} f_{l}(k) f_{\phi}(q)\left(1-f_{N}\left(t, \omega_{\mathbf{p}}\right)\right)\right. \\
\left.-|M(N \rightarrow l \phi)|^{2} f_{N}\left(t, \omega_{\mathbf{p}}\right)\left(1-f_{l}(k)\right)\left(1+f_{\phi}(q)\right)\right]
\end{aligned}
$$

where now $\mathcal{O}\left(\lambda^{4}\right)$ corrections to the matrix elements have to be kept. Using Eq. (2.7) one obtains for the lepton asymmetry

$$
f_{L i}(t, k)=f_{l i}(t, k)-f_{\overline{l i}}(t, k)
$$

with initial condition $f_{L i}(0, k)=0$,

$$
f_{L i}(t, k)=-\epsilon_{i i} \frac{1}{k} \int_{\mathbf{q}, \mathbf{p}}(2 \pi)^{4} \delta^{4}(k+q-p) p \cdot k f_{l \phi}(k, q) f_{N}^{e q}\left(\omega_{\mathbf{p}}\right) \frac{1}{\Gamma}\left(1-e^{-\Gamma t}\right)
$$

where we have defined

$$
\epsilon_{i j}=\frac{3}{16 \pi} \operatorname{Im}\left\{\lambda_{i 1}^{*}\left(\eta \lambda^{*}\right)_{j 1}\right\} M
$$


Summing over all lepton flavours, the generated lepton asymmetry is proportional to the familiar CP asymmetry [9],

$$
\epsilon=\sum_{i} \frac{\epsilon_{i i}}{\left(\lambda^{\dagger} \lambda\right)_{11}}=\frac{3}{16 \pi} \frac{\operatorname{Im}\left(\lambda^{\dagger} \eta \lambda^{*}\right)_{11} M}{\left(\lambda^{\dagger} \lambda\right)_{11}}
$$

For later comparison with solutions of the Kadanoff-Baym equations, it is convenient to rewrite Eq. (2.11) as a 4-fold integral,

$$
\begin{aligned}
f_{L i}(t, k)=-\epsilon_{i i} \frac{16 \pi}{k} & \int_{\mathbf{q}, \mathbf{p}, \mathbf{q}^{\prime}, \mathbf{k}^{\prime}} k \cdot k^{\prime}(2 \pi)^{4} \delta^{4}(k+q-p)(2 \pi)^{4} \delta^{4}\left(k^{\prime}+q^{\prime}-p\right) \\
& \times f_{l \phi}(k, q) f_{N}^{e q}\left(\omega_{\mathbf{p}}\right) \frac{1}{\Gamma}\left(1-e^{-\Gamma t}\right) .
\end{aligned}
$$

The integrand is now proportional to the averaged matrix element $|M(l \phi \rightarrow \bar{l} \bar{\phi})|^{2}=$ $2 k \cdot k^{\prime}\left(\lambda^{\dagger} \lambda\right)_{11} / M^{2}$ (cf. [9]), which involves the product of the 4-vectors $k$ and $k^{\prime}$. At low temperatures, $T \ll M$, the integrand falls off like $e^{-\beta \omega_{\mathbf{p}}}<e^{-\beta M}$, i.e., the generated

asymmetry is strongly suppressed. In standard leptogenesis calculations one considers the integrated lepton asymmetry,

$$
n_{L}=\sum_{i} \int \frac{d^{3} k}{(2 \pi)^{3}} f_{L i}(t, k)
$$

The number densities $n_{N}$ (2.4) and $n_{L}$ correspond to the comoving number densities $\mathrm{N}_{\mathrm{N}_{1}}$ and $\left|\mathrm{N}_{\mathrm{B}-\mathrm{L}}\right|$ shown in Fig. 1, in the initial phase of the time evolution, i.e., for $T \gtrsim 0.3 \mathrm{M}$.

\section{Nonequilibrium QFT and Kadanoff-Baym equations}

In the following, we briefly introduce concepts and quantities from nonequilibrium quantum field theory that are necessary for our computation (cf. [36,37]). A thermodynamical system is represented by a statistical ensemble described by a density matrix $\varrho$. The expectation value for an operator $\mathcal{A}$ is then given by

$$
\langle\mathcal{A}\rangle=\operatorname{Tr}(\varrho \mathcal{A})
$$

where we have adopted the usual normalisation $\operatorname{Tr} \varrho=1$. Solving the initial value problem for $\varrho$ allows to compute all observables for all times. Direct computation of the time evolution of $\varrho$ is difficult. Generically, the von Neumann (or quantum Liouville) equation of motion for $\varrho$ can only be solved perturbatively for a reduced density matrix with an effective Hamiltonian. In most practical applications to date, a number of additional 
assumptions are made that lead to effective Boltzmann equations, which can take account of coherent oscillations, or quantum corrected Boltzmann equations (cf. Section 6) 3 .

Instead of the time evolution of the density matrix, one can also directly study the equations of motion of the correlation functions of the theory. The infinitely many degrees of freedom of the initial density matrix are then mapped onto their infinitely many initial conditions. Though a full characterisation of the system in principle involves all $n$-point functions, it is often sufficient to study the one- and two-point function. This applies to the problem considered in this work.

\subsection{Correlation functions for lepton and Higgs fields}

Leptogenesis occurs at temperatures above the electroweak scale where sphaleron processes are active and transfer the generated lepton asymmetry to a baryon asymmetry. Hence, the Standard Model is in the symmetric phase and the four real degrees of freedom of the Higgs doublet correspond to four massless real scalar fields.

The spectral function and statistical propagator of a real scalar field $\phi, \Delta^{-}$and $\Delta^{+}$, respectively, are defined as

$$
\begin{aligned}
& \Delta^{-}\left(x_{1}, x_{2}\right)=i\left\langle\left[\phi\left(x_{1}\right), \phi\left(x_{2}\right)\right]\right\rangle, \\
& \Delta^{+}\left(x_{1}, x_{2}\right)=\frac{1}{2}\left\langle\left\{\phi\left(x_{1}\right), \phi\left(x_{2}\right)\right\}\right\rangle .
\end{aligned}
$$

Here only contributions from connected diagrams are to be included to compute the dressed correlation functions. These fulfill the symmetry relations

$$
\begin{aligned}
& \Delta^{-}\left(x_{1}, x_{2}\right)=-\Delta^{-}\left(x_{2}, x_{1}\right) \\
& \Delta^{+}\left(x_{1}, x_{2}\right)=\Delta^{+}\left(x_{2}, x_{1}\right)
\end{aligned}
$$

which follow directly from the definitions.

The functions $\Delta^{ \pm}$have an intuitive physical interpretation. The spectral function $\Delta^{-}$ is the Fourier transform of the spectral density,

$$
\rho_{\mathbf{q}}(t, \omega)=-i \int \frac{d y}{2 \pi} e^{i \omega y} \Delta^{-}\left(t+\frac{y}{2}, t-\frac{y}{2}\right),
$$

where we have used the relative and total time coordinates, $y=t_{1}-t_{2}$ and $t=\left(t_{1}+t_{2}\right) / 2$, respectively.

The spectral density $\rho_{\mathbf{q}}(t, \omega)$ characterises the density of quantum mechanical states in phase space. Propagating states, or resonances, appear as peaks in the spectral function.

\footnotetext{
${ }^{2}$ See [38, 39] for an application to neutrino oscillations.

${ }^{3}$ In [40,41] an approach based on first principles has been suggested that is applicable if the occupation numbers for the out-of-equilibrium fields are small.
} 
The statistical propagator contains the information about the occupation number of each state.

In the following we shall also need the Wightman functions

$$
\begin{aligned}
& \Delta^{>}\left(x_{1}, x_{2}\right)=\left\langle\phi\left(x_{1}\right) \phi\left(x_{2}\right)\right\rangle, \\
& \Delta^{<}\left(x_{1}, x_{2}\right)=\left\langle\phi\left(x_{2}\right) \phi\left(x_{1}\right)\right\rangle,
\end{aligned}
$$

which are related to $\Delta^{ \pm}$by

$$
\begin{aligned}
& \Delta^{-}\left(x_{1}, x_{2}\right)=i\left(\Delta^{>}\left(x_{1}, x_{2}\right)-\Delta^{<}\left(x_{1}, x_{2}\right)\right), \\
& \Delta^{+}\left(x_{1}, x_{2}\right)=\frac{1}{2}\left(\Delta^{>}\left(x_{1}, x_{2}\right)+\Delta^{<}\left(x_{1}, x_{2}\right)\right) .
\end{aligned}
$$

Using microcausality and the condition for canonical quantization,

$$
\begin{aligned}
& {\left.\left[\phi\left(x_{1}\right), \phi\left(x_{2}\right)\right]\right|_{t_{1}=t_{2}}=\left.\left[\dot{\phi}\left(x_{1}\right), \dot{\phi}\left(x_{2}\right)\right]\right|_{t_{1}=t_{2}}=0,} \\
& {\left.\left[\phi\left(x_{1}\right), \dot{\phi}\left(x_{2}\right)\right]\right|_{t_{1}=t_{2}}=i \delta\left(\mathbf{x}_{1}-\mathbf{x}_{2}\right),}
\end{aligned}
$$

one obtains boundary conditions in $y=t_{1}-t_{2}$ for $\Delta^{-}$,

$$
\begin{aligned}
\left.\Delta^{-}\left(x_{1}, x_{2}\right)\right|_{t_{1}=t_{2}} & =0 \\
\left.\partial_{t_{1}} \Delta^{-}\left(x_{1}, x_{2}\right)\right|_{t_{1}=t_{2}} & =-\left.\partial_{t_{2}} \Delta^{-}\left(x_{1}, x_{2}\right)\right|_{t_{1}=t_{2}}=\delta\left(\mathbf{x}_{1}-\mathbf{x}_{2}\right) \\
\left.\partial_{t_{1}} \partial_{t_{2}} \Delta^{-}\left(x_{1}, x_{2}\right)\right|_{t_{1}=t_{2}} & =0 .
\end{aligned}
$$

Note that these conditions do not depend on the physical initial conditions of the system encoded in the initial density matrix. These enter via the initial conditions for the statistical propagator.

Analogous to $\Delta^{ \pm}$, one can define the spectral functions and statistical propagators for fermions. The fermionic fields in the Lagrangian (1.2) are massless left-handed leptons (Weyl fields $l_{L i}$ ) and a massive neutrino (Majorana field $N$ ). For the massless leptons, spectral function and statistical propagator are defined as

$$
\begin{aligned}
& \left(S_{L i j}^{-}\right)_{\alpha \beta}\left(x_{1}, x_{2}\right)=i\left\langle\left\{l_{L i \alpha}\left(x_{1}\right), \bar{l}_{L j \beta}\left(x_{2}\right)\right\}\right\rangle, \\
& \left(S_{L i j}^{+}\right)_{\alpha \beta}\left(x_{1}, x_{2}\right)=\frac{1}{2}\left\langle\left[l_{L i \alpha}\left(x_{1}\right), \bar{l}_{L j \beta}\left(x_{2}\right)\right]\right\rangle,
\end{aligned}
$$

where $\alpha$ and $\beta$ are spinor indices, and $\mathrm{SU}(2)$ indices were omitted for notational simplicity. The subscript $L$ denotes the projection to left-handed fields, i.e., $S_{L}^{ \pm}=P_{L} S^{ \pm}$, where $P_{L}=\left(1-\gamma^{5}\right) / 2$ and $S^{ \pm}$are the propagators for Dirac fermions. As for bosons, we shall need the functions

$$
\begin{aligned}
& \left(S_{L i j}^{>}\right)_{\alpha \beta}\left(x_{1}, x_{2}\right)=\left\langle l_{L i \alpha}\left(x_{1}\right) \bar{l}_{L j \beta}\left(x_{2}\right)\right\rangle, \\
& \left(S_{L i j}^{<}\right)_{\alpha \beta}\left(x_{1}, x_{2}\right)=-\left\langle\bar{l}_{L j \beta}\left(x_{2}\right) l_{L i \alpha}\left(x_{1}\right)\right\rangle,
\end{aligned}
$$


which are related to spectral function and statistical propagator by

$$
\begin{aligned}
& S_{L i j}^{-}\left(x_{1}, x_{2}\right)=i\left(S_{L i j}^{>}\left(x_{1}, x_{2}\right)-S_{L i j}^{<}\left(x_{1}, x_{2}\right)\right), \\
& S_{L i j}^{+}\left(x_{1}, x_{2}\right)=\frac{1}{2}\left(S_{L i j}^{>}\left(x_{1}, x_{2}\right)+S_{L i j}^{<}\left(x_{1}, x_{2}\right)\right) .
\end{aligned}
$$

The propagators $S^{ \pm}$have the symmetry properties

$$
\begin{aligned}
& \gamma_{0}\left[S_{L i j}^{-}\left(x_{1}, x_{2}\right)\right]^{\dagger} \gamma_{0}=-S_{L j i}^{-}\left(x_{2}, x_{1}\right), \\
& \gamma_{0}\left[S_{L i j}^{+}\left(x_{1}, x_{2}\right)\right]^{\dagger} \gamma_{0}=S_{L j i}^{+}\left(x_{2}, x_{1}\right) .
\end{aligned}
$$

The canonical quantization condition,

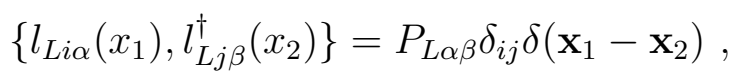

implies the boundary condition for the spectral function

$$
\left.S_{L i j}^{-}\left(x_{1}, x_{2}\right)\right|_{t_{1}=t_{2}}=i P_{L} \delta_{i j} \delta\left(\mathbf{x}_{1}-\mathbf{x}_{2}\right) .
$$

Finally, spectral function and statistical propagator for the Majorana field $N$ read

$$
\begin{aligned}
& G_{\alpha \beta}^{-}\left(x_{1}, x_{2}\right)=i\left\langle\left\{N_{\alpha}\left(x_{1}\right), N_{\beta}\left(x_{2}\right)\right\}\right\rangle, \\
& G_{\alpha \beta}^{+}\left(x_{1}, x_{2}\right)=\frac{1}{2}\left\langle\left[N_{\alpha}\left(x_{1}\right), N_{\beta}\left(x_{2}\right)\right]\right\rangle .
\end{aligned}
$$

They have the symmetries

$$
\begin{aligned}
& G^{-}\left(x_{1}, x_{2}\right)=G^{-}\left(x_{2}, x_{1}\right)^{T}, \\
& G^{+}\left(x_{1}, x_{2}\right)=-G^{+}\left(x_{2}, x_{1}\right)^{T} .
\end{aligned}
$$

The canonical quantization condition, together with the Majorana property $N=C \bar{N}^{T}$, implies the boundary condition

$$
\left.G^{-}\left(x_{1}, x_{2}\right)\right|_{t_{1}=t_{2}}=i \gamma^{0} \delta\left(\mathbf{x}_{1}-\mathbf{x}_{2}\right) C^{-1} .
$$

As for scalars, the physical initial conditions enter as boundary conditions for the statistical propagator. In the following, we will consider two types of initial conditions, thermal equilibrium and Gaussian initial correlations, for which we solve the equations of motion in the following section. Analogous to real scalars, the functions $G^{\gtrless}$ are defined as

$$
\begin{aligned}
& G_{\alpha \beta}^{>}\left(x_{1}, x_{2}\right)=\left\langle N_{\alpha}\left(x_{1}\right) N_{\beta}\left(x_{2}\right)\right\rangle, \\
& G_{\alpha \beta}^{<}\left(x_{1}, x_{2}\right)=-\left\langle N_{\beta}\left(x_{2}\right) N_{\alpha}\left(x_{1}\right)\right\rangle,
\end{aligned}
$$

with the usual relations to spectral function and statistical propagator,

$$
\begin{aligned}
& G^{-}\left(x_{1}, x_{2}\right)=i\left(G^{>}\left(x_{1}, x_{2}\right)-G^{<}\left(x_{1}, x_{2}\right)\right), \\
& G^{+}\left(x_{1}, x_{2}\right)=\frac{1}{2}\left(G^{>}\left(x_{1}, x_{2}\right)+G^{<}\left(x_{1}, x_{2}\right)\right) .
\end{aligned}
$$




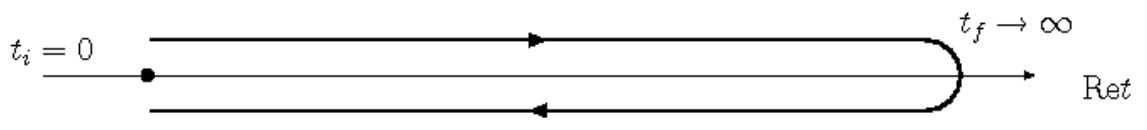

Figure 2: Path in the complex time plane for nonequilibrium Green's functions. The contour runs from some initial time $x^{0}=t_{i}+i \epsilon\left(t_{i}=0\right)$ parallel to the real axis $\left(x^{0}=t+i \epsilon\right)$ up to some final time $t_{f}+i \epsilon$ and returns to $t_{i}-i \epsilon$. To compute physical correlation functions for arbitrary times $t>t_{i}$, one takes the limits $t_{f} \rightarrow \infty$ and $\epsilon \rightarrow 0$.

\subsection{Equations of motion}

In thermal leptogenesis, the deviation from thermal equilibrium that is necessary to create a matter-antimatter asymmetry is due to the heavy Majorana neutrinos which are out of equilibrium. The equations of motion for their correlation functions $G^{ \pm}$can be obtained via the Schwinger-Keldysh formalism [6]. The basic quantity is the Green's function with time arguments defined on a contour $\mathcal{C}$ in the complex $x^{0}$-plane, known as the Keldysh contour (cf. Fig,2),

$$
G_{\mathcal{C}}\left(x_{1}, x_{2}\right)=\theta_{\mathcal{C}}\left(x_{1}^{0}, x_{2}^{0}\right) G^{>}\left(x_{1}, x_{2}\right)+\theta_{\mathcal{C}}\left(x_{2}^{0}, x_{1}^{0}\right) G^{<}\left(x_{1}, x_{2}\right) .
$$

Here the $\theta$-functions enforce path ordering along the contour $\mathcal{C}$. The necessity of considering Green's functions with time arguments on the Keldysh contour (rather than the real axis) is a consequence of the fact that nonequilibrium processes are initial value problems. The system is prepared at initial time $t_{i}$, its state at later times is unknown. Hence, the usual approach to define a S-matrix by projection onto asymptotic 'in' and 'out' states, sending initial and final time to infinity, cannot be applied. When using the Keldysh contour which starts and ends at the same time $t_{t}^{4}$, no knowledge of the system's state at $t= \pm \infty$ is needed to define a generating functional for correlation functions.

The Green's function $G_{\mathcal{C}}$ satisfies the Schwinger-Dyson equation

$$
C\left(i \not \partial_{1}-M\right) G_{\mathcal{C}}\left(x_{1}, x_{2}\right)-i \int_{\mathcal{C}} d^{4} x^{\prime} C \Sigma_{\mathcal{C}}\left(x_{1}, x^{\prime}\right) G_{\mathcal{C}}\left(x^{\prime}, x_{2}\right)=i \delta_{\mathcal{C}}\left(x_{1}-x_{2}\right)
$$

where $C \Sigma_{\mathcal{C}}\left(x_{1}, x^{\prime}\right)$ is the self-energy $5^{5}$ on the contour and $\partial_{1}=\gamma^{\mu} \partial / \partial x_{1}^{\mu}$. Like the Green's function, also the self-energy can be decomposed as

$$
\Sigma_{\mathcal{C}}\left(x_{1}, x_{2}\right)=\theta_{\mathcal{C}}\left(x_{1}^{0}, x_{2}^{0}\right) \Sigma^{>}\left(x_{1}, x_{2}\right)+\theta_{\mathcal{C}}\left(x_{2}^{0}, x_{1}^{0}\right) \Sigma^{<}\left(x_{1}, x_{2}\right) .
$$

\footnotetext{
${ }^{4}$ Due to this fact this formalism is sometimes called 'in-in' formalism, in contrast to the 'in-out' formalism used to compute the S-matrix.

${ }^{5}$ An explicit factor $C$ is factorized for later convenience.
} 
In the Schwinger-Dyson equation (3.36) the time coordinates of $G_{\mathcal{C}}$ and $\Sigma_{\mathcal{C}}$ can lie on the upper or the lower branch of the contour.

The familar time-ordered Feynman propagator is obtained from $G_{\mathcal{C}}\left(x_{1}, x_{2}\right)$ when both time arguments lie on the upper branch, and therefore denoted by $G^{11}$. Correspondingly, $G_{\mathcal{C}}\left(x_{1}, x_{2}\right)$ with both time arguments on the lower part of the contour corresponds to an anti-time-ordered propagator, denoted as $G^{22}$. For correlators with one time argument on the upper and one on the lower part of the contour, referred to as $G^{12}$ and $G^{21}$, the order of field operators is fixed by the path ordering: operators on the upper branch are always 'earlier' than those on the lower branch (cf. 3.35). Altogether, one has

$$
\begin{aligned}
& G^{12}\left(x_{1}, x_{2}\right)=G^{<}\left(x_{1}, x_{2}\right), \\
& G^{21}\left(x_{1}, x_{2}\right)=G^{>}\left(x_{1}, x_{2}\right), \\
& G^{11}\left(x_{1}, x_{2}\right)=G^{+}\left(x_{1}, x_{2}\right)-\frac{i}{2} \operatorname{sign}\left(x_{1}^{0}-x_{2}^{0}\right) G^{-}\left(x_{1}, x_{2}\right), \\
& G^{22}\left(x_{1}, x_{2}\right)=G^{+}\left(x_{1}, x_{2}\right)+\frac{i}{2} \operatorname{sign}\left(x_{1}^{0}-x_{2}^{0}\right) G^{-}\left(x_{1}, x_{2}\right) ;
\end{aligned}
$$

the last two relations are easily verified by inserting the definitions of $G^{ \pm}$.

In a perturbative expansion of the Schwinger-Dyson equation (3.36) in terms of Feynman diagrams, time arguments of internal vertices can lie on either branch. Hence, the number of contributing graphs doubles with each internal vertex since this can lie on the upper or the lower branch 6 . Two upper vertices are connected by $G^{11}$, two lower vertices by $G^{22}$ and vertices of different type by $G^{12}$ and $G^{21}$. Each lower vertex leads to an additional factor -1 .

Like the Green's function, also the self-energy $\Sigma_{\mathcal{C}}$, the sum of all one-particle irreducible graphs, can be dissected into components $\Sigma^{k l}$, with $k$ and $l$ being 'contour indices' as defined above. Analogous to (3.38) and (3.39) one then defines self-energies $\Sigma \gtrless$ and, following (3.33) and (3.34), self-energies $\Sigma^{ \pm}$via the equations

$$
\begin{aligned}
& \Sigma^{-}\left(x_{1}, x_{2}\right)=i\left(\Sigma^{>}\left(x_{1}, x_{2}\right)-\Sigma^{<}\left(x_{1}, x_{2}\right)\right), \\
& \Sigma^{+}\left(x_{1}, x_{2}\right)=\frac{1}{2}\left(\Sigma^{>}\left(x_{1}, x_{2}\right)+\Sigma^{<}\left(x_{1}, x_{2}\right)\right) .
\end{aligned}
$$

Since the self-energies $\Sigma^{k l}$ are directly related to the full Green's functions $G^{k l}$, they also satisfy the relations (3.38) - (3.41).

Using the above relations for $G^{k l}$ and $\Sigma^{k l}$, one obtains, after a straightforward calculation, from the Schwinger-Dyson equation (3.36) a system of two coupled differential equations for $G_{\mathbf{p}}^{ \pm}$, the Kadanoff-Baym equations. Due to spatial homogeneity, we can consider the equations for each Fourier mode separately,

$$
C\left(i \gamma^{0} \partial_{t_{1}}-\mathbf{p} \gamma-M\right) G_{\mathbf{p}}^{-}\left(t_{1}, t_{2}\right)=-\int_{t_{1}}^{t_{2}} d t^{\prime} C \Sigma_{\mathbf{p}}^{-}\left(t_{1}, t^{\prime}\right) G_{\mathbf{p}}^{-}\left(t^{\prime}, t_{2}\right)
$$

\footnotetext{
${ }^{6}$ This fact is sometimes referred to as 'doubling of degrees of freedom'.
} 


$$
\begin{aligned}
C\left(i \gamma^{0} \partial_{t_{1}}-\mathbf{p} \gamma-M\right) G_{\mathbf{p}}^{+}\left(t_{1}, t_{2}\right)= & -\int_{t_{i}}^{t_{2}} d t^{\prime} C \Sigma_{\mathbf{p}}^{+}\left(t_{1}, t^{\prime}\right) G_{\mathbf{p}}^{-}\left(t^{\prime}, t_{2}\right) \\
& +\int_{t_{i}}^{t_{1}} d t^{\prime} C \Sigma_{\mathbf{p}}^{-}\left(t_{1}, t^{\prime}\right) G_{\mathbf{p}}^{+}\left(t^{\prime}, t_{2}\right)
\end{aligned}
$$

For the lepton propagators $S_{L \mathbf{k}}^{ \pm}$one obtains the same equations, with $C \Sigma_{\mathbf{p}}^{ \pm}$replaced by the lepton self-energies $\Pi_{\mathrm{k}}^{ \pm}$and no charge conjugation matrix $\mathrm{C}$ multiplying the kinetic term.

The Kadanoff-Baym equations (3.44) and (3.45) are exact. They contain all quantum and non-Markovian effects including the dependence on the initial time $t_{i}$. Furthermore, in contrast to usual linear response techniques, they do not rely on any assumption regarding the size of the initial deviation from equilibrium. The equations in this form are valid for arbitrary nonequilibrium initial states which can be parameterized by Gaussian initial correlations. This covers the case considered in this work since the generated lepton asymmetry involves to leading order in the Yukawa coupling only the 2-point functions of the heavy neutrino. When higher order initial correlations play a significant role, the Kadanoff-Baym formalism is still applicable, but the equation for the statistical propagator contains extra terms at $t_{i}$ [42]. In [36], thermalization has been studied for a scalar field theory using the equation of motion for the statistical propagator.

In nonequilibrium quantum field theory, instead of distribution functions, quantum mechanical correlation functions $G^{ \pm}$characterise the state of the system. The interactions enter via the self-energies $\Sigma^{ \pm}$which, via the generalized cutting rules, contain all possible processes. Encoding this information in the self-energies avoids potential problems related to the definition of asymptotic states for unstable particles as well as the substraction of real intermediate state contributions in Boltzmann equations. Note, finally, that the integro-differential equations (3.44), (3.45) do not suffer from the late time uncertainties or secular terms that perturbative expansions of Boltzmann equations are often plagued with when applied to multiscale problems (cf. [36]).

\subsection{Weak coupling to a thermal bath}

The Kadanoff-Baym equations provide a tool to study the dynamics of arbitrary nonequilibrium systems. Unfortunately, in most cases they can only be solved numerically. As discussed in the introduction, in this work we consider a rather simple system: one field that is out of equilibrium $(N)$ is weakly coupled to a large thermal bath of Standard Model fields. This leads to a number of simplifications compared to the general case that allow to find analytic solutions. We have previously studied scalar field models of this type [29,43]. Here we extend the methods developed therein to the case of thermal leptogenesis.

The Standard Model interactions keep the bath in thermal equilibrium. The corresponding time scale $\tau_{S M} \sim 1 /\left(g^{2} T\right)$ at temperature $T \sim M$ is much shorter than the equilibration time $\tau_{N} \sim 1 /\left(\lambda^{2} M\right)$ of the heavy neutrino, which governs the generation of the lepton asymmetry: $\tau_{S M} \ll \tau_{N}$. Lepton number changing processes in the thermal bath 

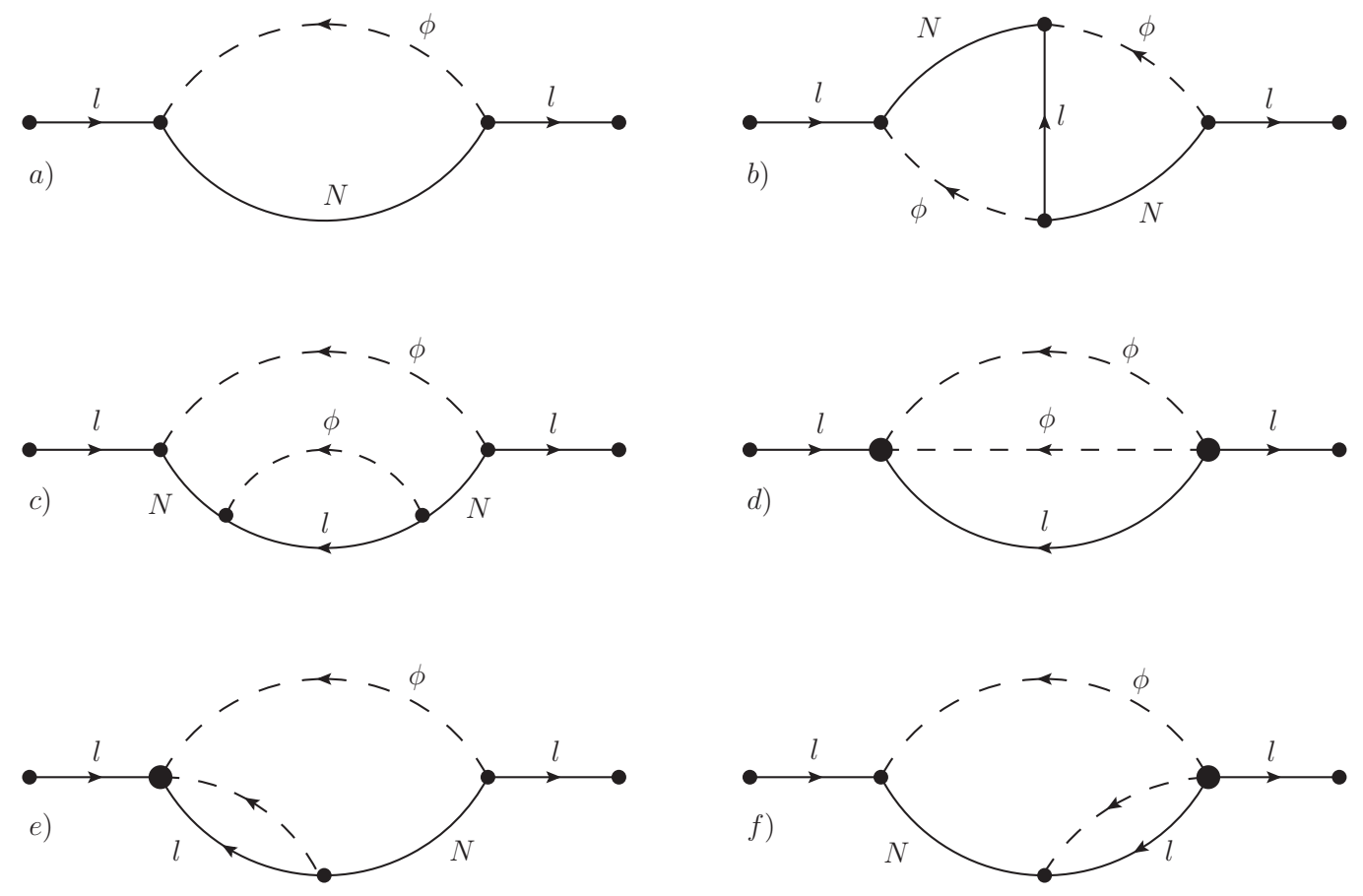

Figure 3: One- and two-loop contributions to the lepton self-energy correspoding to washout terms, a) - d), and $\mathrm{CP}$ violating terms which generate a lepton asymmetry, e) and $\mathrm{f}$ ).

are shown in Fig. 3. As in the case of Boltzmann equations discussed in Section 2, we focus on the $\mathrm{CP}$ violating interaction generating the lepton asymmetry that correspond to Fig. [3 e) and f).

To evaluate these graphs we need the correlation functions of lepton and Higgs fields in the thermal bath. A system in thermal equilibrium is described by the density matrix

$$
\varrho_{e q}=\frac{\exp \left(\beta\left(-\mathcal{H}+\mu_{i} \mathcal{Q}_{i}\right)\right)}{\operatorname{Tr} \exp \left(\beta\left(-\mathcal{H}+\mu_{i} \mathcal{Q}_{i}\right)\right)},
$$

where $\mathcal{H}$ is the Hamiltonian of the system, $\beta$ is the inverse temperature, $\mathcal{Q}_{i}$ are conserved charges and $\mu_{i}$ are the corresponding chemical potentials. As expected for an initial state after inflation, we set all chemical potentials equal to zero.

Equilibrium correlation functions of a spatially homogeneous system only depend on space-time differences, and it is convenient to consider the Fourier transforms,

$$
\begin{aligned}
& \Delta_{\mathbf{q}}^{ \pm}(\omega)=\int d^{4} x e^{i\left(\omega x^{0}-\mathbf{q x}\right)} \Delta^{ \pm}(x), \\
& S_{\mathbf{k}}^{ \pm}(\omega)=\int d^{4} x e^{i\left(\omega x^{0}-\mathbf{k x}\right)} S^{ \pm}(x) .
\end{aligned}
$$




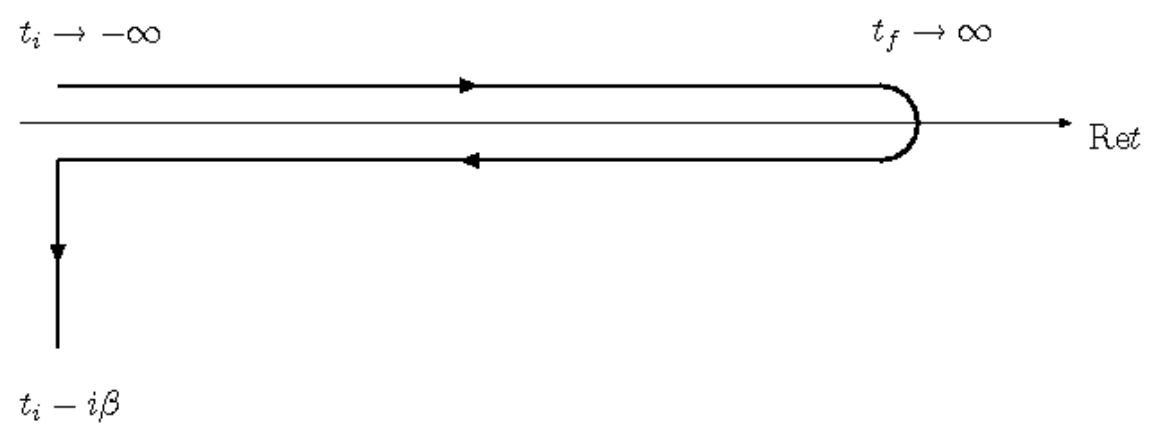

Figure 4: Path $\mathcal{C}_{\beta}$ in the complex time plane for equilibrium correlation functions.

The equilibrium density matrix (3.46) then corresponds to a shift in imaginary time. This leads to the well-known Kubo-Martin-Schwinger (KMS) relations (cf. [44])

$$
\Delta_{\mathbf{q}}^{<}(\omega)=e^{-\beta \omega} \Delta_{\mathbf{q}}^{>}(\omega), \quad S_{\mathbf{k}}^{<}(\omega)=-e^{-\beta \omega} S_{\mathbf{k}}^{>}(\omega),
$$

which imply

$$
\begin{aligned}
& \Delta_{\mathbf{q}}^{+}(\omega)=-i\left(\frac{1}{2}+f_{\phi}(\omega)\right) \Delta_{\mathbf{q}}^{-}(\omega)=-\frac{i}{2} \operatorname{coth}\left(\frac{\beta \omega}{2}\right) \Delta_{\mathbf{q}}^{-}(\omega), \\
& S_{\mathbf{k}}^{+}(\omega)=-i\left(\frac{1}{2}-f_{l}(\omega)\right) S_{\mathbf{k}}^{-}(\omega)=-\frac{i}{2} \tanh \left(\frac{\beta \omega}{2}\right) S_{\mathbf{k}}^{-}(\omega),
\end{aligned}
$$

where

$$
f_{\phi}(\omega)=\frac{1}{e^{\beta \omega}-1}, \quad f_{l}(\omega)=\frac{1}{e^{\beta \omega}+1},
$$

are Bose-Einstein and Fermi-Dirac distribution functions, respectively. Note that the en$\operatorname{ergy} \omega$ is not on-shell.

Equilibrium Green's functions can be calculated in the real-time formalism using the contour $\mathcal{C}_{\beta}$ in the complex time plane, which is shown in Fig. 4. For the free equilibrium propagators of massless lepton and Higgs fields one obtains $(q=|\mathbf{q}|, k=|\mathbf{k}|$, cf. [44]),

$$
\begin{aligned}
\Delta_{\mathbf{q}}^{-}(y) & =\frac{1}{q} \sin (q y), \\
\Delta_{\mathbf{q}}^{+}(y) & =\frac{1}{2 q} \operatorname{coth}\left(\frac{\beta q}{2}\right) \cos (q y), \\
S_{L \mathbf{k}}^{-}(y) & =P_{L}\left(i \gamma_{0} \cos (k y)-\frac{\mathbf{k} \gamma}{k} \sin (k y)\right), \\
S_{L \mathbf{k}}^{+}(y) & =-\frac{1}{2} P_{L} \tanh \left(\frac{\beta k}{2}\right)\left(i \gamma_{0} \sin (k y)+\frac{\mathbf{k} \gamma}{k} \cos (k y)\right) .
\end{aligned}
$$




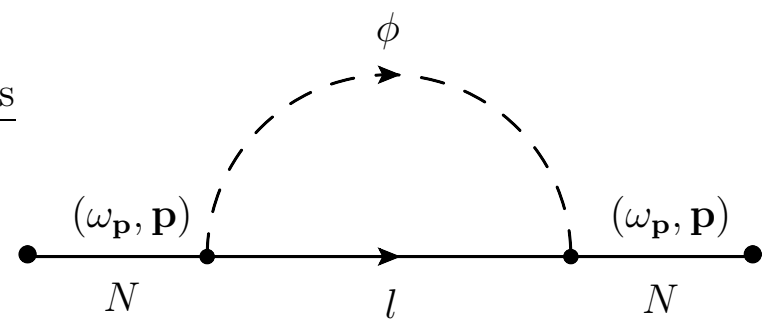

Figure 5: One-loop contribution to the self-energies $C \Sigma_{\mathbf{p}}^{ \pm}$of the Majorana neutrino $N$.

All other propagators can be obtained as linear combinations using the relations described in the previous paragraph. A complete list is given in Appendix A.

In the following sections we shall see that the calculation of the lepton asymmetry represents an initial value problem which can be treated based on the real time formalism together with the Keldysh contour Fig. 2. Thermal and nonthermal properties of the system are then encoded in the initial values of the various Green's functions.

\section{Nonequilibrium correlation functions}

The assumption of weak coupling to a large thermal bath with negligible backreaction in the framework of Kadanoff-Baym equations implies that self-energies for the heavy neutrinos $N$ are computed from equilibrium propagators of bath fields only. This also corresponds to a leading order perturbative expansion in the coupling constant.

Perturbative expansions of Boltzmann equations in multiscale problems are known to suffer from uncertainties, so-called secular terms, at late times. The Kadanoff-Baym equations (3.44) and (3.45) in full generality are free of secular terms and consistently include all memory effects. Nevertheless, the neglect of backreaction in the computation of $\Sigma$ corresponds to a truncation in the perturbative expansion in the Yukawa couplings $\lambda$, which might introduce similar uncertainties related to the multiscale nature of the problem. However, in the system of consideration contributions of higher order in $\lambda$ are not only suppressed by the smallness of the coupling, but also by the number of degrees of freedom in the bath that justify the neglect of backreaction. Hence, we expect potential problems due to secular terms not to be relevant.

The assumption that the background medium equilibrates instantaneously on the time scale of the asymmetry generation leaves open the details of the equilibration process. In reality, there are effects related to the finite equilibration time and the finite size of the quasi-particles. As we shall see in Section 5, these quantities play a crucial role in the Kadanoff-Baym result for the lepton asymmetry.

The self-energy for the heavy neutrino $N$ to leading order in $\lambda$ is given by the diagram in Fig. 5. It contains time-translation invariant propagators of bath fields only, and hence it is also time-translation invariant. As shown in [29], this implies that also the spectral 
function is time-translation invariant, $G_{\mathbf{p}}^{-}\left(t_{1}, t_{2}\right) \equiv G_{\mathbf{p}}^{-}(y), y=t_{1}-t_{2}$. In this case we can find the general solutions to the Kadanoff-Baym equations without further approximations.

\subsection{Equation for the spectral function}

Let us now consider the equation for the spectral function of the Majorana neutrino. After an obvious change of variables, the Kadanoff-Baym equation (3.44) becomes,

$$
C\left(i \gamma^{0} \partial_{y}-\mathbf{p} \gamma-M\right) G_{\mathbf{p}}^{-}(y)-\int_{0}^{y} d y^{\prime} C \Sigma_{\mathbf{p}}^{-}\left(y-y^{\prime}\right) G_{\mathbf{p}}^{-}\left(y^{\prime}\right)=0
$$

Defining the Laplace transform

$$
\tilde{G}_{\mathbf{p}}^{-}(s)=\int_{0}^{\infty} d y e^{-s y} G_{\mathbf{p}}^{-}(y), \quad \tilde{\Sigma}_{\mathbf{p}}^{-}(s)=\int_{0}^{\infty} d y e^{-s y} \Sigma_{\mathbf{p}}^{-}(y),
$$

one obtains from Eq. (4.1)

$$
\left(i \gamma^{0} s-\mathbf{p} \gamma-M-\tilde{\Sigma}_{\mathbf{p}}^{-}(s)\right) \tilde{G}_{\mathbf{p}}^{-}(s)=i \gamma^{0} G_{\mathbf{p}}^{-}(0) .
$$

Using the boundary condition (3.30),

$$
G_{\mathbf{p}}^{-}(0)=i \gamma^{0} C^{-1}
$$

this leads to

$$
\tilde{G}^{-}(s)=-\left(i \gamma^{0} s-\mathbf{p} \gamma-M-\tilde{\Sigma}^{-}(s)\right)^{-1} C^{-1}
$$

The inverse Laplace transform is given by

$$
G_{\mathbf{p}}^{-}(y)=\int_{\mathcal{C}_{B}} \frac{d s}{2 \pi i} e^{s y} \tilde{G}_{\mathbf{p}}^{-}(s)
$$

where $\mathcal{C}_{B}$ is the Bromwich contour (see Fig. 6): The part parallel to the imaginary axis is chosen such that all singularities of the integrand are to its left; the second part is the semicircle at infinity which closes the contour at $\operatorname{Re}(s)<0$.

From the definition of the Laplace transform one can see that the self-energy $\tilde{\Sigma}_{\mathbf{p}}^{-}(s)$ is analytic on the real $s$ axis, but has a discontinuity across the imaginary axis. This gives rise to the spectral representation

$$
\tilde{\Sigma}_{\mathbf{p}}^{-}(s)=i \int_{-\infty}^{\infty} \frac{d p_{0}}{2 \pi} \frac{\Sigma_{\mathbf{p}}^{-}\left(p_{0}\right)}{i s-p_{0}} .
$$

Note that the retarded and advanced self-energies are given by

$$
\tilde{\Sigma}_{\mathbf{p}}^{-}(-i \omega+\epsilon)=\Sigma_{\mathbf{p}}^{R}(\omega)
$$




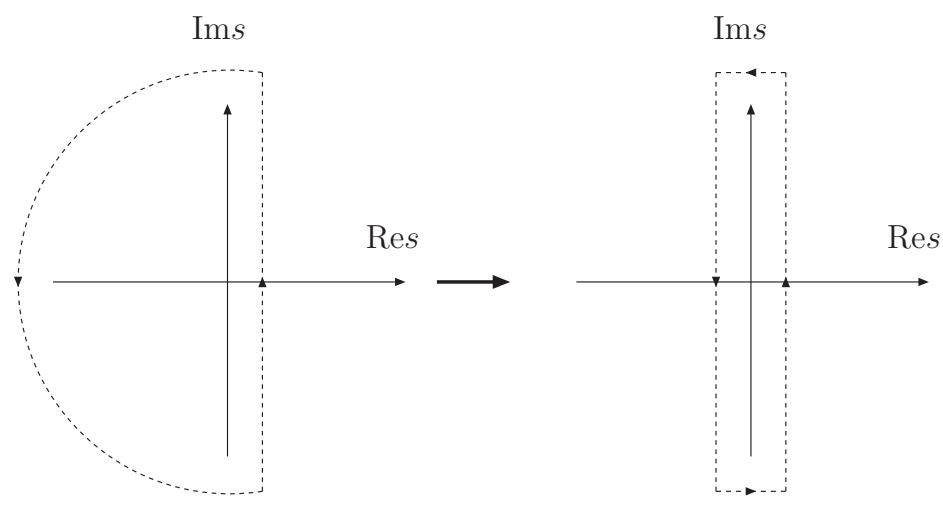

Figure 6: Bromwich contour

$$
\tilde{\Sigma}_{\mathbf{p}}^{-}(-i \omega-\epsilon)=\Sigma_{\mathbf{p}}^{A}(\omega)
$$

These self-energies are determined by the discontinuity of $\tilde{\Sigma}_{\mathbf{p}}^{-}(s)$,

$$
\operatorname{disc} \tilde{\Sigma}_{\mathbf{p}}^{-}(-i \omega)=\tilde{\Sigma}_{\mathbf{p}}^{-}(-i \omega+\epsilon)-\tilde{\Sigma}_{\mathbf{p}}^{-}(-i \omega-\epsilon)=\Sigma_{\mathbf{p}}^{-}(\omega),
$$

with the real part given by the principal value, i.e.,

$$
\tilde{\Sigma}_{\mathbf{p}}^{-}(-i \omega \pm \epsilon)=i \mathcal{P} \int_{-\infty}^{\infty} \frac{d p_{0}}{2 \pi} \frac{\Sigma^{-}\left(p_{0}\right)}{\omega-p_{0}} \pm \frac{1}{2} \Sigma_{\mathbf{p}}^{-}(\omega) \text {. }
$$

This representation of the self-energy is familiar from the theory at zero temperature.

We are now ready to calculate the spectral function in terms of the self-energy $\Sigma_{\mathbf{p}}^{-}(\omega)$. Its Laplace transform has singularities only on the imaginary axis. Hence the Bromwich contour can be deformed as $\mathcal{C}_{B} \rightarrow \int_{-i \infty+\epsilon}^{i \infty+\epsilon}+\int_{i \infty-\epsilon}^{-i \infty-\epsilon}$ (see Fig. 6), which yields for the spectral function

$$
\begin{aligned}
G_{\mathbf{p}}^{-}(y) & =\int_{\mathcal{C}_{B}} \frac{d s}{2 \pi i} e^{s y} \tilde{G}_{\mathbf{p}}^{-}(s) \\
& =\int_{-\infty}^{\infty} \frac{d \omega}{2 \pi} e^{(i \omega+\epsilon) y} \tilde{G}_{\mathbf{p}}^{-}(i \omega+\epsilon)+\int_{\infty}^{-\infty} \frac{d \omega}{2 \pi} e^{(i \omega-\epsilon) y} \tilde{G}_{\mathbf{p}}^{-}(i \omega-\epsilon) \\
& =\int_{-\infty}^{\infty} \frac{d \omega}{2 \pi} e^{-i \omega y}\left(\tilde{G}_{\mathbf{p}}^{-}(-i \omega+\epsilon)-\tilde{G}_{\mathbf{p}}^{-}(-i \omega-\epsilon)\right) .
\end{aligned}
$$

The Fourier transform of the spectral function,

$$
\rho_{\mathbf{p}}(\omega)=-i \int_{-\infty}^{\infty} d y e^{i \omega y} G_{\mathbf{p}}^{-}(y)
$$


is then given by

$$
\rho_{\mathbf{p}}(\omega)=\left(\frac{-i}{\not p-M-\frac{1}{2} \Sigma_{\mathbf{p}}^{-}(\omega)}-\frac{-i}{\not p-M+\frac{1}{2} \Sigma_{\mathbf{p}}^{-}(\omega)}\right) C^{-1} .
$$

Here we have assumed that the divergent contribution of the real part has already been absorbed into mass and wave function renormalization, so that $\rho_{\mathbf{p}}(\omega)$ represents the renormalized spectral density. The finite part of the self-energy is negligable because of the small Yukawa coupling.

A straightforward calculation yields for the self-energy (cf. [35]),

$$
\Sigma_{\mathbf{p}}^{-}(\omega)=2 i\left(\lambda^{\dagger} \lambda\right)_{11} \int_{\mathbf{k}, \mathbf{q}} \not \not \sigma(p ; k, q)
$$

where we have defined

$$
\begin{aligned}
\sigma(p ; k, q)= & f_{l \phi}(k, q)(2 \pi)^{4}\left(\delta^{4}(p-k-q)+\delta^{4}(p+k+q)\right) \\
& +\bar{f}_{l \phi}(k, q)(2 \pi)^{4}\left(\delta^{4}(p+k-q)+\delta^{4}(p-k+q)\right),
\end{aligned}
$$

with the statistical factors

$$
f_{l \phi}(k, q)=1-f_{l}(k)+f_{\phi}(q), \quad \bar{f}_{l \phi}(k, q)=f_{\phi}(q)+f_{l}(k) .
$$

Note that $k$ and $q$ are on-shell, i.e., $k=(k, \mathbf{k})$ and $q=(q, \mathbf{q})$, whereas $p=(\omega, \mathbf{p})$ is off-shell. The properties of the Dirac matrices and rotational invariance imply

$$
\Sigma_{\mathbf{p}}^{-}(\omega)=i a_{\mathbf{p}}(\omega) \gamma^{0}+i b_{\mathbf{p}}(\omega) \mathbf{p} \gamma
$$

where

$$
\begin{aligned}
& a_{\mathbf{p}}(\omega)=2\left(\lambda^{\dagger} \lambda\right)_{11} \int_{\mathbf{q}, \mathbf{k}} k \sigma(p ; k, q) \\
& b_{\mathbf{p}}(\omega)=-2\left(\lambda^{\dagger} \lambda\right)_{11} \frac{1}{\mathbf{p}^{2}} \int_{\mathbf{q}, \mathbf{k}} \mathbf{p k} \sigma(p ; k, q) .
\end{aligned}
$$

These functions satisfy the relations

$$
a_{\mathbf{p}}\left(-\omega_{\mathbf{p}}\right)=a_{\mathbf{p}}\left(\omega_{\mathbf{p}}\right), \quad b_{\mathbf{p}}\left(-\omega_{\mathbf{p}}\right)=-b_{\mathbf{p}}\left(\omega_{\mathbf{p}}\right) .
$$

Using Eq. (4.18) and linearising the denominators in Eq. (4.14) in the small quantities $a_{\mathbf{p}}(\omega)$ and $b_{\mathbf{p}}(\omega)$, one obtains for the spectral density

$$
\rho_{\mathbf{p}}(\omega)=\frac{2 \omega \Gamma_{\mathbf{p}}(\omega)}{\left(\omega^{2}-\omega_{\mathbf{p}}^{2}\right)+\left(\omega \Gamma_{\mathbf{p}}(\omega)\right)^{2}}(\not p+M) C^{-1},
$$


where

$$
\begin{aligned}
\omega \Gamma_{\mathbf{p}}(\omega) & =\omega a_{\mathbf{p}}(\omega)+\mathbf{p}^{2} b_{\mathbf{p}}(\omega) \\
& =2\left(\lambda^{\dagger} \lambda\right)_{11} \int_{\mathbf{q}, \mathbf{k}} p \cdot k \sigma(p ; k, q) .
\end{aligned}
$$

On-shell, only the first of the $\delta$-functions in $\sigma(p ; k, q)$ contributes, and one obtains the width appearing in the Boltzmann equations,

$$
\Gamma_{\mathbf{p}}\left(\omega_{\mathbf{p}}\right)=\left(\lambda^{\dagger} \lambda\right)_{11} \frac{2}{\omega_{\mathbf{p}}} \int_{\mathbf{q}, \mathbf{k}} p \cdot k f_{l \phi}(k, q)(2 \pi)^{4} \delta^{4}(p-k-q) \equiv \Gamma_{\mathbf{p}}
$$

which satisfies the relations

$$
\Gamma_{\mathbf{p}}\left(-\omega_{\mathbf{p}}\right)=\Gamma_{-\mathbf{p}}\left(\omega_{\mathbf{p}}\right)=\Gamma_{\mathbf{p}}\left(\omega_{\mathbf{p}}\right)
$$

In the zero-width limit the spectral function (4.22) reduces to the familiar expression in vacuum,

$$
\rho_{\mathbf{p}}(\omega)=2 \pi \operatorname{sign}(\omega) \delta\left(p^{2}-M^{2}\right)(\not p+M) C^{-1} .
$$

The spectral propagator is now obtained by evaluating the Fourier transform of the spectral function (4.22),

$$
G_{\mathbf{p}}^{-}(y)=i \int_{-\infty}^{\infty} \frac{d \omega}{2 \pi} e^{-i \omega y} \rho_{\mathbf{p}}(\omega)
$$

which yields the final result

$$
G_{\mathbf{p}}^{-}(y)=\left(i \gamma_{0} \cos \left(\omega_{\mathbf{p}} y\right)+\frac{M-\mathbf{p} \gamma}{\omega_{\mathbf{p}}} \sin \left(\omega_{\mathbf{p}} y\right)\right) e^{-\Gamma_{\mathbf{p}}|y| / 2} C^{-1} .
$$

Compared to the free spectral function only an exponential damping factor appears. This is a feature of the narrow-width approximation, analogous to the scalar field case discussed in [29].

\subsection{Equation for the statistical propagator}

We now proceed to the solution of the second Kadanoff-Baym equation (3.45) which, choosing $t_{i}=0$, reads

$$
C\left(i \gamma^{0} \partial_{t_{1}}-\mathbf{p} \gamma-M\right) G_{\mathbf{p}}^{+}\left(t_{1}, t_{2}\right)-\int_{0}^{t_{1}} d t^{\prime} C \Sigma_{\mathbf{p}}^{-}\left(t_{1}-t^{\prime}\right) G_{\mathbf{p}}^{+}\left(t^{\prime}, t_{2}\right)=\zeta_{\mathbf{p}}\left(t_{1}-t_{2}\right)
$$


with the source term

$$
\zeta_{\mathbf{p}}\left(t_{1}-t_{2}\right)=-\int_{0}^{t_{2}} d t^{\prime} \Sigma_{\mathbf{p}}^{+}\left(t_{1}-t^{\prime}\right) G_{\mathbf{p}}^{-}\left(t^{\prime}-t_{2}\right) .
$$

The general solution of (4.29) takes the form

$$
G_{\mathbf{p}}^{+}\left(t_{1}, t_{2}\right)=\hat{G}_{\mathbf{p}}^{+}\left(t_{1}, t_{2}\right)+G_{\mathbf{p}, \text { mem }}^{+}\left(t_{1}, t_{2}\right),
$$

where $\hat{G}_{\mathbf{p}}^{+}\left(t_{1}, t_{2}\right)$ is the general solution of the homogeneous equation

$$
C\left(i \gamma^{0} \partial_{t_{1}}-\mathbf{p} \gamma-M\right) \hat{G}_{\mathbf{p}}^{+}\left(t_{1}, t_{2}\right)-\int_{0}^{t_{1}} d t^{\prime} C \Sigma_{\mathbf{p}}^{-}\left(t_{1}-t^{\prime}\right) \hat{G}_{\mathbf{p}}^{+}\left(t^{\prime}, t_{2}\right)=0
$$

and the 'memory integral', which contains non-Markovian effects, is given by

$$
G_{\mathbf{p}, \text { mem }}^{+}\left(t_{1}, t_{2}\right)=\int_{0}^{t_{1}} d t^{\prime} \int_{0}^{t_{2}} d t^{\prime \prime} G_{\mathbf{p}}^{-}\left(t_{1}-t^{\prime}\right) \Sigma_{\mathbf{p}}^{+}\left(t^{\prime}-t^{\prime \prime}\right) G_{\mathbf{p}}^{-}\left(t^{\prime \prime}-t_{2}\right) .
$$

One easily verifies that the memory integral is a special solution of the inhomogeneous equation.

In order to evaluate the memory integral we perform a Fourier transform of the selfenergy $\left(y=t_{1}-t_{2}\right)$,

$$
\begin{aligned}
& G_{\mathbf{p}, \text { mem }}^{+}\left(t_{1}, t_{2}\right)= \\
& \qquad \frac{d \omega}{2 \pi}\left(\int_{0}^{t_{1}} d y_{1} G_{\mathbf{p}}^{-}\left(y_{1}\right) e^{i \omega y_{1}}\right) \Sigma_{\mathbf{p}}^{+}(\omega)\left(\int_{0}^{t_{2}} d y_{2} G_{\mathbf{p}}^{-}\left(-y_{2}\right) e^{-i \omega y_{2}}\right) e^{-i \omega y} .
\end{aligned}
$$

Since the self-energy is computed with fields in thermal equilibrium, it satisfies the KMS condition (cf. (3.51) $)$

$$
\Sigma_{\mathbf{p}}^{+}(\omega)=-\frac{i}{2} \tanh \left(\frac{\beta \omega}{2}\right) \Sigma_{\mathbf{p}}^{-}(\omega) .
$$

Using the expressions (4.18) and (4.28) for self-energy and spectral function, respectively, which were derived in the previous section, it is now straightforward to calculate the memory integral explicitly. Neglecting terms $\mathcal{O}\left(\Gamma_{\mathbf{p}}\right)$ in the numerator, one finds

$$
\begin{aligned}
\int_{0}^{t} d y e^{i \omega y} G_{\mathbf{p}}^{-}(y) & =\frac{1}{\omega_{\mathbf{p}}^{2}-\left(\omega+i \Gamma_{\mathbf{p}} / 2\right)^{2}} \times \\
& \left(i \gamma^{0}\left[\left(\omega_{\mathbf{p}} \sin \left(\omega_{\mathbf{p}} t\right)+i \omega \cos \left(\omega_{\mathbf{p}} t\right)\right) e^{i\left(\omega+i \Gamma_{\mathbf{p}} / 2\right) t}-i \omega\right]\right. \\
& \left.+\frac{M-\mathbf{p} \gamma}{\omega_{\mathbf{p}}}\left[i \omega\left(\sin \left(\omega_{\mathbf{p}} t\right)-\omega_{\mathbf{p}} \cos \left(\omega_{\mathbf{p}} t\right)\right) e^{i\left(\omega+i \Gamma_{\mathbf{p}} / 2\right) t}+\omega_{\mathbf{p}}\right]\right) C^{-1}
\end{aligned}
$$




$$
\begin{aligned}
\int_{0}^{t} d y e^{-i \omega y} G_{\mathbf{p}}^{-}(-y) & =\frac{1}{\omega_{\mathbf{p}}^{2}-\left(\omega-i \Gamma_{\mathbf{p}} / 2\right)^{2}} \times \\
& \left(i \gamma^{0}\left[\left(\omega_{\mathbf{p}} \sin \left(\omega_{\mathbf{p}} t\right)-i \omega \cos \left(\omega_{\mathbf{p}} t\right)\right) e^{-i\left(\omega-i \Gamma_{\mathbf{p}} / 2\right) t}-i \omega\right]\right. \\
& \left.+\frac{M-\mathbf{p} \gamma}{\omega_{\mathbf{p}}}\left[i \omega\left(\sin \left(\omega_{\mathbf{p}} t\right)-\omega_{\mathbf{p}} \cos \left(\omega_{\mathbf{p}} t\right)\right) e^{i\left(\omega+i \Gamma_{\mathbf{p}} / 2\right) t}+\omega_{\mathbf{p}}\right]\right) C^{-1}
\end{aligned}
$$

After inserting these expressions in Eq. 4.34 one can perform the $\omega$-integration using Cauchy's theorem. The integrand has two poles 7 in the upper-half plane at $\omega=i \Gamma_{\mathbf{p}} / 2 \pm \omega_{\mathbf{p}}$, and two poles in the lower-half plane $\omega=-i \Gamma_{\mathbf{p}} / 2 \pm \omega_{\mathbf{p}}$. The choice of the contour depends on the sign of the time variables in the exponent. The result is a sum of the contributions from all four poles. The expressions appearing in the numerator can be simplified by means of Eqs. (4.21) and (4.24) for self-energy and equilibration width, respectively,

$$
\begin{aligned}
\left(\gamma^{0}+\frac{M-\mathbf{p} \gamma}{\omega_{\mathbf{p}}}\right) \Sigma_{\mathbf{p}}^{-}\left(\omega_{\mathbf{p}}\right)\left(\gamma^{0}+\frac{M-\mathbf{p} \gamma}{\omega_{\mathbf{p}}}\right) & =2 i \Gamma_{\mathbf{p}}\left(\gamma^{0}+\frac{M-\mathbf{p} \gamma}{\omega_{\mathbf{p}}}\right) \\
\left(\gamma^{0}-\frac{M-\mathbf{p} \gamma}{\omega_{\mathbf{p}}}\right) \Sigma_{\mathbf{p}}^{-}\left(-\omega_{\mathbf{p}}\right)\left(\gamma^{0}-\frac{M-\mathbf{p} \gamma}{\omega_{\mathbf{p}}}\right) & =2 i \Gamma_{\mathbf{p}}\left(\gamma^{0}-\frac{M-\mathbf{p} \gamma}{\omega_{\mathbf{p}}}\right) .
\end{aligned}
$$

Using these expressions one finally obtains for the memory integral, changing variables from $\left(t_{1}, t_{2}\right)$ to $(t, y)$,

$$
\begin{aligned}
& G_{\mathbf{p}, \text { mem }}^{+}(t, y)= \\
& -\frac{1}{2} \tanh \left(\frac{\beta \omega_{\mathbf{p}}}{2}\right)\left(i \gamma_{0} \sin \left(\omega_{\mathbf{p}} y\right)-\frac{M-\mathbf{p} \boldsymbol{\gamma}}{\omega_{\mathbf{p}}} \cos \left(\omega_{\mathbf{p}} y\right)\right)\left(e^{-\Gamma_{\mathbf{q}}|y| / 2}-e^{-\Gamma_{\mathbf{q}} t}\right) C^{-1} .
\end{aligned}
$$

Asymptotically, for $t \rightarrow \infty$, the memory integral becomes

$$
G_{\mathbf{p}}^{+ \text {eq }}(t, y)=-\frac{1}{2} \tanh \left(\frac{\beta \omega_{\mathbf{p}}}{2}\right)\left(i \gamma_{0} \sin \left(\omega_{\mathbf{p}} y\right)-\frac{M-\mathbf{p} \boldsymbol{\gamma}}{\omega_{\mathbf{p}}} \cos \left(\omega_{\mathbf{p}} y\right)\right) e^{-\Gamma_{\mathbf{q}}|y| / 2} C^{-1}
$$

One easily verifies that $G_{\mathbf{p}}^{+\mathrm{eq}}(t, y)$ indeed represents the equilibrium statistical propagator. For the Fourier transform one obtains

$$
\begin{aligned}
G_{\mathbf{p}}^{+\mathrm{eq}}(\omega) & =\int_{-\infty}^{\infty} d y e^{i \omega y} G_{\mathbf{p}}^{+\mathrm{eq}}(y) \\
& =\frac{1}{2} \tanh \left(\frac{\beta \omega}{2}\right) \frac{2 \omega \Gamma_{\mathbf{p}}(\omega)}{\left(\omega^{2}-\omega_{\mathbf{p}}^{2}\right)+\left(\omega \Gamma_{\mathbf{p}}(\omega)\right)^{2}}(\not p+M) C^{-1} \\
& =\frac{1}{2} \tanh \left(\frac{\beta \omega}{2}\right) \rho_{\mathbf{p}}(\omega)
\end{aligned}
$$

\footnotetext{
${ }^{7}$ There are further poles at $\omega_{n}= \pm i \pi(1+2 n) / \beta, n$ integer. However, their contribution to $G_{\mathbf{p}, \text { mem }}^{+}$is $\mathcal{O}\left(\Gamma_{\mathbf{p}} / M\right)$ and therefore negligible.
} 


$$
=-\frac{i}{2} \tanh \left(\frac{\beta \omega}{2}\right) G_{\mathbf{p}}^{-}(\omega)
$$

i.e., the KMS condition (cf. (3.51)) is indeed satisfied.

In order to obtain the general solution of the inhomogeneous Kadanoff-Baym equation we have to add to the memory integral the general solution of the homogeneous equation (4.32). This equation is identical to the Kadanoff-Baym equation for the spectral function (4.1) with $t_{2}$ playing the role of an additional parameter. Hence, the functional dependence of $\hat{G}_{\mathbf{p}}^{+}\left(t_{1}, t_{2}\right)$ on the first argument $t_{1}$ can be obtained in the same way as for the spectral function. Applying the Laplace transform to (4.32) one finds

$$
\tilde{G}_{\mathbf{p}}^{+}\left(s, t_{2}\right)=\frac{1}{i \gamma_{0} s-\mathbf{p} \gamma-M-\tilde{\Sigma}^{-}(s)} i \gamma_{0} \hat{G}_{\mathbf{p}}^{+}\left(0, t_{2}\right)
$$

The inverse Laplace transform then gives

$$
\hat{G}_{\mathbf{p}}^{+}\left(t_{1}, t_{2}\right)=-G_{\mathbf{p}}^{-}\left(t_{1}\right) C i \gamma_{0} \hat{G}_{\mathbf{p}}^{+}\left(0, t_{2}\right)
$$

The function $\hat{G}_{\mathbf{p}}^{+}\left(0, t_{2}\right)$ can now be determined by the symmetries (3.28) and (3.29) of $\hat{G}_{\mathbf{p}}^{ \pm}\left(t_{1}, t_{2}\right)$, which imply

$$
\hat{G}_{\mathbf{p}}^{+}\left(t_{1}, t_{2}\right)^{T}=-\hat{G}_{\mathbf{p}}^{+}\left(t_{2}, t_{1}\right) .
$$

This yields the result

$$
\hat{G}_{\mathbf{p}}^{+}\left(t_{1}, t_{2}\right)=-G_{\mathbf{p}}^{-}\left(t_{1}\right) C \gamma_{0} G_{\mathbf{p}}^{+}(0,0) \gamma_{0} C^{-1} G_{\mathbf{p}}^{-}\left(-t_{2}\right)
$$

where $G_{\mathbf{p}}^{+}(0,0)$ is an antisymmetric matrix.

Let us first consider the case of thermal initial condition,

$$
G_{\mathbf{p}}^{+\mathrm{eq}}(0,0)=\frac{M-\mathbf{p} \boldsymbol{\gamma}}{2 \omega_{\mathbf{p}}} \tanh \left(\frac{\beta \omega}{2}\right) C^{-1}
$$

From Eq. (4.47) one then obtains

$$
\hat{G}_{\mathbf{p}}^{+\mathrm{eq}}\left(t_{1}, t_{2}\right)=-\frac{1}{2}\left(i \gamma_{0} \sin \left(\omega_{\mathbf{p}} y\right)-\frac{M-\mathbf{p} \boldsymbol{\gamma}}{\omega_{\mathbf{p}}} \cos \left(\omega_{\mathbf{p}} y\right)\right) \tanh \left(\frac{\beta \omega_{\mathbf{p}}}{2}\right) e^{-\Gamma_{\mathbf{q}}\left(t_{1}+t_{2}\right) / 2} C^{-1}
$$

Adding this expression to the memory integral $\hat{G}_{\mathbf{p}, \text { mem }}^{+}$one obtains the equilibrium statistical propagator $\hat{G}_{\mathbf{p}}^{+ \text {eq }}$ which is independent of $t=\left(t_{1}+t_{2}\right) / 2$. Hence, as expected, the equilibrium statistical propagator is a solution of the full Kadanoff-Baym equation. 
We are particularly interested in the case of vacuum initial condition, which corresponds to zero initial abundance for heavy neutrinos in the Boltzmann case. The vacuum propagators are obtained from the equilibrium ones in the limit $\beta \rightarrow \infty$. Hence we choose

$$
G_{\mathbf{p}}^{+\operatorname{vac}}(0,0)=\frac{M-\mathbf{p} \gamma}{2 \omega_{\mathbf{p}}} C^{-1}
$$

From Eqs. (4.31), (4.40) and (4.47) one then obtains the full solution for the statistical propagator, which interpolates between vacuum at $t=0$ and equilibrium for $t \rightarrow \infty$,

$$
\begin{aligned}
G_{\mathbf{p}}^{+}(t, y)= & -\left(i \gamma_{0} \sin \left(\omega_{\mathbf{p}} y\right)-\frac{M-\mathbf{p} \gamma}{\omega_{\mathbf{p}}} \cos \left(\omega_{\mathbf{p}} y\right)\right) \\
& \times\left[\frac{1}{2} \tanh \left(\frac{\beta \omega_{\mathbf{p}}}{2}\right) e^{-\Gamma_{\mathbf{p}}|y| / 2}+f_{N}^{e q}\left(\omega_{\mathbf{p}}\right) e^{-\Gamma_{\mathbf{p}} t}\right] C^{-1} .
\end{aligned}
$$

This result will be the basis for the calculation of the lepton asymmetry in the next section. All heavy neutrino propagators can be obtained as linear combinations of the spectral function $G_{\mathbf{p}}^{-}(y)$ and the statistical propagator $G_{\mathbf{p}}^{+}(t, y)$. A full list is given in Appendix A.

Finally, let us emphasize that the solution of the Kadanoff-Baym equation for the statistical propagator is not related to the equilibrium propagator by a simple change of the distribution function from $f_{N}^{\mathrm{eq}}(\omega)$ to some nonequilibrium function $f_{N}^{\mathrm{eq}}(t, \omega)$. This is in contrast to the assumption made in the derivation of Quantum Boltzman equations [16, 17, 19, 21]. For a system close to equilibrium this assumption leads to a valid approximation of the Kadanoff-Baym equations [9], but in general it is not justified.

\section{$5 \quad$ Lepton asymmetries}

We are now ready to calculate the lepton asymmetry which is generated during the approach of the heavy Majorana neutrino $N$ to thermal equilibrium. Our starting point is the flavour non-diagonal lepton current, which is obtained from the statistical propagator,

$$
j_{i j}^{\mu}(x)=-\operatorname{tr}\left[\gamma^{\mu} S_{L i j}^{+}\left(x, x^{\prime}\right)\right]_{x^{\prime} \rightarrow x} .
$$

Since we consider a spatially homogeneous system, $S_{i j}^{+}\left(x, x^{\prime}\right)$ only depends on the difference $\vec{x}-\vec{x}^{\prime}$, and it is convenient to perform a Fourier transform. The zeroth component of the current, the 'lepton number matrix', is given by

$$
L_{\mathbf{k} i j}\left(t, t^{\prime}\right)=-\operatorname{tr}\left[\gamma_{0} S_{L \mathbf{k} i j}^{+}\left(t, t^{\prime}\right)\right] .
$$

One easily verifies that for free fields in equilibrium

$$
L_{\mathbf{k} i i}(t, t)=f_{l i}(k)-f_{\overline{l i}}(k),
$$




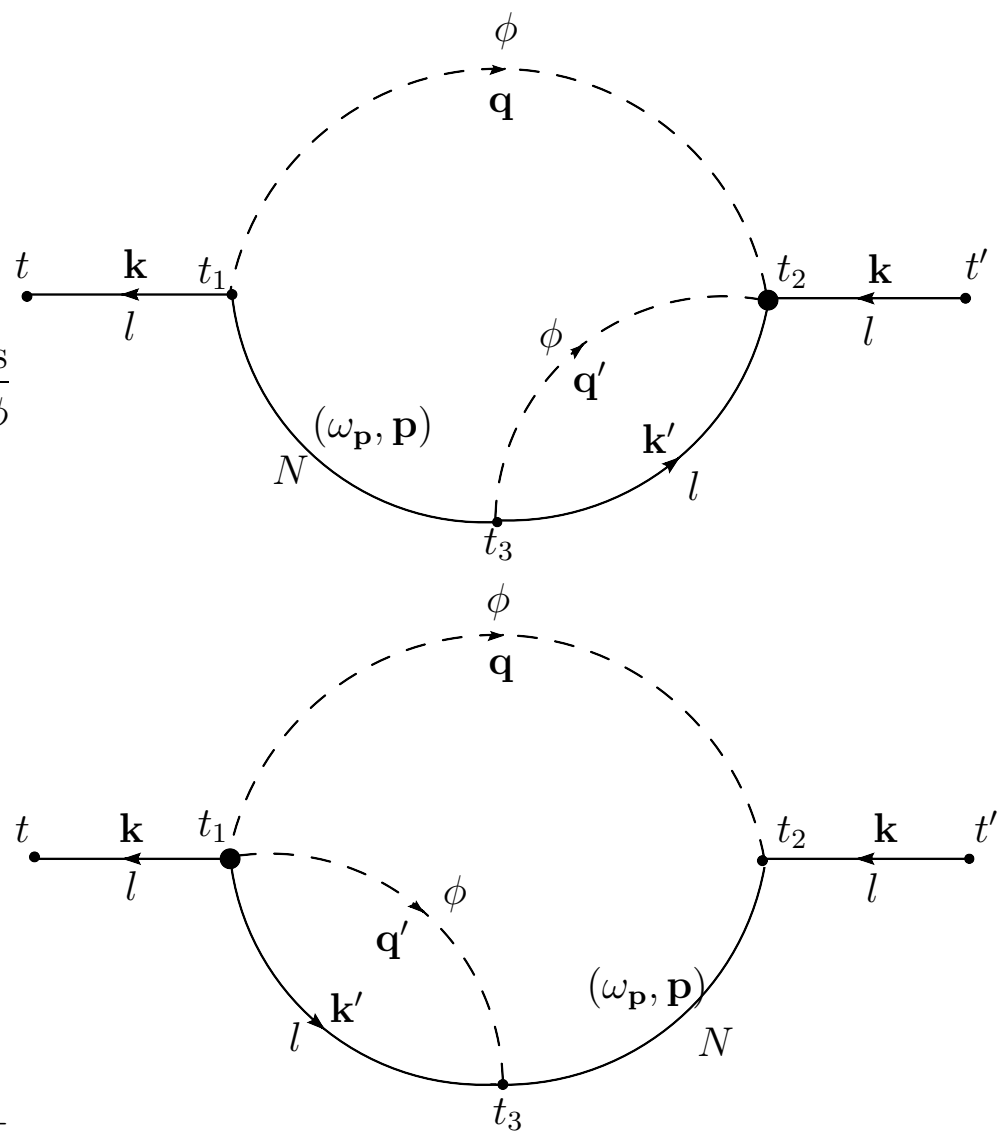

Figure 7: Two-loop contributions to the lepton self-energies $\Pi_{\mathbf{k}}^{ \pm}$, which lead to a nonzero lepton number densities.

where $f_{l i}$ and $f_{\overline{l i}}$ are the distribution functions of leptons and anti-leptons, respectively.

The lepton number matrix $L_{\mathbf{k} i j}\left(t, t^{\prime}\right)$ can be directly computed from the self-energy corrections to the statistical propagator shown in Fig. 3, the external lepton couples to Majorana neutrino and Higgs boson, and also to Higgs boson and Higgs-lepton pair. Complex Yukawa couplings and quantum interference then lead to a non-vanishing lepton asymmetry.

For a homogeneous system, the Kadanoff-Baym equation for the statistical propagator (cf. (3.45) ) yields for each Fourier mode the equations

$$
\begin{aligned}
\left(i \gamma^{0} \partial_{t}-\mathbf{k} \gamma\right) S_{L \mathbf{k}}^{+}\left(t, t^{\prime}\right)= & \int_{0}^{t} d t_{1} \Pi_{\mathbf{k}}^{-}\left(t, t_{1}\right) S_{L \mathbf{k}}^{+}\left(t_{1}, t^{\prime}\right) \\
& -\int_{0}^{t^{\prime}} d t_{1} \Pi_{\mathbf{k}}^{+}\left(t, t_{1}\right) S_{L \mathbf{k}}^{-}\left(t_{1}, t^{\prime}\right)
\end{aligned}
$$




$$
\begin{aligned}
S_{L \mathbf{k}}^{+}\left(t, t^{\prime}\right)\left(-i \gamma^{0} \overleftarrow{\partial_{t^{\prime}}}-\mathbf{k} \gamma\right)= & -\int_{0}^{t^{\prime}} d t_{1} S_{L \mathbf{k}}^{+}\left(t, t_{1}\right) \Pi_{\mathbf{k}}^{-}\left(t_{1}, t^{\prime}\right) \\
& +\int_{0}^{t} d t_{1} S_{L \mathbf{k}}^{-}\left(t, t_{1}\right) \Pi_{\mathbf{k}}^{+}\left(t_{1}, t^{\prime}\right)
\end{aligned}
$$

One then obtains for the time derivative of the lepton number matrix, dropping flavour indices (cf. [18]) 8

$$
\begin{aligned}
& \partial_{t} L_{\mathbf{k}}(t, t)= i \operatorname{tr}\left[\left(i \gamma_{0} \partial_{t}+i \gamma_{0} \partial_{t^{\prime}}\right) S_{L \mathbf{k}}^{+}\left(t, t^{\prime}\right)\right]_{t=t^{\prime}} \\
&= i \operatorname{tr}\left[\left(i \gamma_{0} \partial_{t}-\mathbf{k} \gamma\right) S_{L \mathbf{k}}^{+}\left(t, t^{\prime}\right)+S_{L \mathbf{k}}^{+}\left(t, t^{\prime}\right)\left(i \gamma^{0} \overleftarrow{\partial_{t^{\prime}}}+\mathbf{k} \gamma\right)\right]_{t=t^{\prime}} \\
&=i \operatorname{tr}\left[\int_{0}^{t} d t_{1} \Pi_{\mathbf{k}}^{-}\left(t, t_{1}\right) S_{L \mathbf{k}}^{+}\left(t_{1}, t^{\prime}\right)-\int_{0}^{t^{\prime}} d t_{1} \Pi_{\mathbf{k}}^{+}\left(t, t_{1}\right) S_{L \mathbf{k}}^{-}\left(t_{1}, t^{\prime}\right)\right. \\
& \\
&\left.+\int_{0}^{t^{\prime}} d t_{1} S_{L \mathbf{k}}^{+}\left(t, t_{1}\right) \Pi_{\mathbf{k}}^{-}\left(t_{1}, t^{\prime}\right)-\int_{0}^{t} d t_{1} S_{L \mathbf{k}}^{-}\left(t, t_{1}\right) \Pi_{\mathbf{k}}^{+}\left(t_{1}, t^{\prime}\right)\right]_{t=t^{\prime}}
\end{aligned}
$$

Using properties of the trace and the identity between integration domains

$$
\int_{0}^{t} d t_{1} \int_{0}^{t_{1}} d t_{2} \cdots+\int_{0}^{t} d t_{2} \int_{0}^{t_{2}} d t_{1} \cdots=\int_{0}^{t} d t_{1} \int_{0}^{t} d t_{2} \cdots
$$

one finds

$$
L_{\mathbf{k}}(t, t)=i \int_{0}^{t} d t_{1} \int_{0}^{t} d t_{2} \operatorname{tr}\left[\Pi_{\mathbf{k}}^{-}\left(t_{1}, t_{2}\right) S_{L \mathbf{k}}^{+}\left(t_{2}, t_{1}\right)-\Pi_{\mathbf{k}}^{+}\left(t_{1}, t_{2}\right) S_{L \mathbf{k}}^{-}\left(t_{2}, t_{1}\right)\right] .
$$

Note that $\Pi_{\mathbf{k}}^{ \pm}$and $S_{\mathbf{k}}^{ \pm}$are self-energies and propagators of the full theory including gauge interactions of lepton and Higgs fields.

Using the relations for propagators and self-energies

$$
\begin{aligned}
S_{L \mathbf{k}}^{+} & =\frac{1}{2}\left(S_{L \mathbf{k}}^{>}+S_{L \mathbf{k}}^{<}\right), \quad S_{L \mathbf{k}}^{-}=i\left(S_{L \mathbf{k}}^{>}-S_{L \mathbf{k}}^{<}\right), \\
\Pi_{\mathbf{k}}^{+} & =\frac{1}{2}\left(\Pi_{\mathbf{k}}^{>}+\Pi_{\mathbf{k}}^{<}\right), \quad \Pi_{\mathbf{k}}^{-}=i\left(\Pi_{\mathbf{k}}^{>}-\Pi_{\mathbf{k}}^{<}\right),
\end{aligned}
$$

one obtains from Eq. (5.8) an equivalent useful expression for the lepton number matrix,

$$
L_{\mathbf{k}}(t, t)=-\int_{0}^{t} d t_{1} \int_{0}^{t} d t_{2} \operatorname{tr}\left[\Pi_{\mathbf{k}}^{>}\left(t_{1}, t_{2}\right) S_{L \mathbf{k}}^{<}\left(t_{2}, t_{1}\right)-\Pi_{\mathbf{k}}^{<}\left(t_{1}, t_{2}\right) S_{L \mathbf{k}}^{>}\left(t_{2}, t_{1}\right)\right] .
$$

We want to calculate the lepton asymmetry to leading order in the small Yukawa coupling $\lambda$, which can be achieved in a perturbative expansion. For the heavy neutrino propagator appearing in the loop, the departure from the equilibrium propagator is important 9

${ }^{8}$ We thank C. Weniger for helpful discussions.

${ }^{9}$ We show in Appendix D that the equilibrium part of the propagator does indeed not contribute to the asymmetry. 
which has been evaluated in the previous section,

$$
G_{\mathbf{p}}\left(t_{1}, t_{2}\right)=G_{\mathbf{p}}^{\mathrm{eq}}\left(t_{1}-t_{2}\right)+\bar{G}_{\mathbf{p}}\left(t_{1}, t_{2}\right)
$$

Lepton propagators and self-energies have large equilibrium contributions dominated by gauge interaction, with small corrections $\mathcal{O}\left(\lambda^{2}\right)$,

$$
\begin{aligned}
S_{L \mathbf{k}}\left(t_{1}, t_{2}\right) & =S_{L \mathbf{k}}^{\mathrm{eq}}\left(t_{1}-t_{2}\right)+\delta S_{L \mathbf{k}}\left(t_{1}, t_{2}\right), \\
\Pi_{\mathbf{k}}\left(t_{1}, t_{2}\right) & =\Pi_{\mathbf{k}}^{\mathrm{eq}}\left(t_{1}-t_{2}\right)+\delta \Pi_{\mathbf{k}}\left(t_{1}, t_{2}\right),
\end{aligned}
$$

which include CP-violating source terms and washout terms. Clearly, inserting $\Pi_{\mathbf{k}}^{\mathrm{eq}}$ and $S_{\mathbf{k}}^{\mathrm{eq}}$ in Eq. (5.8) must yield $L_{\mathbf{k}}^{\mathrm{eq}}(t, t)=0$, since no asymmetry is generated in thermal equilibrium 10 As discussed in Section 2, we also neglect washout terms for simplicity. One then obtains for the lepton number matrix $L_{\mathbf{k}}(t, t)$ to leading order in $\lambda$,

$$
\begin{aligned}
& L_{\mathbf{k}}(t, t)=i \int_{0}^{t} d t_{1} \int_{0}^{t} d t_{2} \operatorname{tr}\left[\delta \Pi_{\mathbf{k}}^{-}\left(t_{1}, t_{2}\right) S_{L \mathbf{k}}^{\mathrm{eq}+}\left(t_{2}-t_{1}\right)\right. \\
&\left.-\delta \Pi_{\mathbf{k}}^{+}\left(t_{1}, t_{2}\right) S_{L \mathbf{k}}^{\mathrm{eq}-}\left(t_{2}-t_{1}\right)\right] .
\end{aligned}
$$

Here $\delta \Pi_{\mathbf{k}}$ is given by the two-loop graphs shown in Fig. 17, which have to be evaluated with equilibrium propagators for lepton and Higgs fields and the nonequilibrium Majorana neutrino propagator.

The equilibrium propagators with standard model gauge interactions remain to be evaluated. In the quasi-particle approximation one simply replaces energies $k$ by complex quasi-particle energies $\Omega_{\mathbf{k}}=\left(\mathbf{k}^{2}+m_{\mathrm{th}}^{2}\right)^{1 / 2}+i \gamma(k)$. In the following we shall consider two approximations: free equilibrium propagators with zero chemical potential as given in Eqs. (A.1), (A.2) and (A.7), (A.8),

$$
\Delta_{\mathbf{k}}^{\mathrm{eq} \pm}(y)=\Delta_{\mathbf{k}}^{ \pm}(y), \quad S_{L \mathbf{k}}^{\mathrm{eq} \pm}(y)=S_{L \mathbf{k}}^{ \pm}(y)
$$

and, as a rough approximation to full thermal propagators, free equilibrium propagators modified by thermal damping rates,

$$
\Delta_{\mathbf{k}}^{\mathrm{eq} \pm}(y)=\Delta_{\mathbf{k}}^{ \pm}(y) e^{-\gamma_{\Phi}|y|}, \quad S_{L \mathbf{k}}^{\mathrm{eq} \pm}(y)=S_{L \mathbf{k}}^{ \pm}(y) e^{-\gamma_{l}|y|}
$$

Remarkably, thermal widths turn out to be qualitatively more important than thermal masses, as we shall explain in Section 6.

The two contributions to the self-energy $\delta \Pi_{\mathbf{k} i j}$ (cf. Fig. 7),

$$
\delta \Pi_{\mathbf{k} i j}\left(t_{1}, t_{2}\right)=\Pi_{\mathbf{k} i j}^{(1)}\left(t_{1}, t_{2}\right)+\Pi_{\mathbf{k} i j}^{(2)}\left(t_{1}, t_{2}\right),
$$

\footnotetext{
${ }^{10}$ Note that thermal equilibrium does not correspond to a Gaussian state [42. Therefore one has to include contributions from $n$-point functions which are not determined by equilibrium 2-point functions. However, such terms do not contribute to leading order in the Yukawa coupling $\lambda$.
} 
factorize into a product of Yukawa couplings, which contains the flavour dependence, and a trace of thermal propagators,

$$
\begin{aligned}
& \Pi_{\mathbf{k} i j}^{(1)}\left(t_{1}, t_{2}\right)=-3 i \lambda_{i 1}^{*}\left(\eta \lambda^{*}\right)_{j 1} \Pi_{\mathbf{k}}^{(1)}\left(t_{1}, t_{2}\right), \\
& \Pi_{\mathbf{k} i j}^{(2)}\left(t_{1}, t_{2}\right)=3 i\left(\eta^{*} \lambda\right)_{i 1} \lambda_{j 1} \Pi_{\mathbf{k}}^{(2)}\left(t_{1}, t_{2}\right) .
\end{aligned}
$$

In the case of free equilibrium propagators for lepton and Higgs fields, we obtain for the self-energies $\Pi_{\mathbf{k}}^{(1,2)>}$ and $\Pi_{\mathbf{k}}^{(1,2)<}$ :

$$
\begin{aligned}
\Pi_{\mathbf{k}}^{(1)>}\left(t_{1}, t_{2}\right)= & \int_{0}^{\infty} d t_{3} \int \frac{d^{3} \mathbf{q}}{(2 \pi)^{3}} \frac{d^{3} \mathbf{q}^{\prime}}{(2 \pi)^{3}} \\
& \times\left[\tilde{G}_{\mathbf{p}}^{>}\left(t_{1}, t_{3}\right) S_{\mathbf{k}^{\prime}}^{11}\left(t_{2}-t_{3}\right) \Delta_{\mathbf{q}^{\prime}}^{11}\left(t_{2}-t_{3}\right) \Delta_{\mathbf{q}}^{<}\left(t_{2}-t_{1}\right)\right. \\
& \left.-\tilde{G}_{\mathbf{p}}^{22}\left(t_{1}, t_{3}\right) S_{\mathbf{k}^{\prime}}^{<}\left(t_{2}-t_{3}\right) \Delta_{\mathbf{q}^{\prime}}^{<}\left(t_{2}-t_{3}\right) \Delta_{\mathbf{q}}^{<}\left(t_{2}-t_{1}\right)\right] P_{L}, \\
\Pi_{\mathbf{k}}^{(1)<}\left(t_{1}, t_{2}\right)= & \int_{0}^{\infty} d t_{3} \int \frac{d^{3} \mathbf{q}}{(2 \pi)^{3}} \frac{d^{3} \mathbf{q}^{\prime}}{(2 \pi)^{3}} \\
& \times\left[\tilde{G}_{\mathbf{p}}^{11}\left(t_{1}, t_{3}\right) S_{\mathbf{k}^{\prime}}^{>}\left(t_{2}-t_{3}\right) \Delta_{\mathbf{q}^{\prime}}^{>}\left(t_{2}-t_{3}\right) \Delta_{\mathbf{q}}^{>}\left(t_{2}-t_{1}\right)\right. \\
& \left.-\tilde{G}_{\mathbf{p}}^{<}\left(t_{1}, t_{3}\right) S_{\mathbf{k}^{\prime}}^{22}\left(t_{2}-t_{3}\right) \Delta_{\mathbf{q}^{\prime}}^{22}\left(t_{2}-t_{3}\right) \Delta_{q}^{>}\left(t_{2}-t_{1}\right)\right] P_{L}, \\
\Pi_{\mathbf{k}}^{(2)>}\left(t_{1}, t_{2}\right)= & \int_{0}^{\infty} d t_{3} \int \frac{d^{3} \mathbf{q}}{(2 \pi)^{3}} \frac{d^{3} \mathbf{q}^{\prime}}{(2 \pi)^{3}} \\
& \times\left[\tilde{G}_{\mathbf{p}}^{<}\left(t_{2}, t_{3}\right) S_{\mathbf{k}^{\prime}}^{22}\left(t_{3}-t_{1}\right) \Delta_{\mathbf{q}^{\prime}}^{22}\left(t_{3}-t_{1}\right) \Delta_{\mathbf{q}}^{<}\left(t_{2}-t_{1}\right)\right. \\
& \left.-\tilde{G}_{\mathbf{p}}^{11}\left(t_{2}, t_{3}\right) S_{\mathbf{k}^{\prime}}^{<}\left(t_{3}-t_{1}\right) \Delta_{\mathbf{q}^{\prime}}^{<}\left(t_{3}-t_{1}\right) \Delta_{\mathbf{q}}^{<}\left(t_{2}-t_{1}\right)\right] P_{L}, \\
\Pi_{\mathbf{k}}^{(2)<}\left(t_{1}, t_{2}\right)= & \int_{0}^{\infty} d t_{3} \int \frac{d^{3} \mathbf{q}}{(2 \pi)^{3}} \frac{d^{3} \mathbf{q}^{\prime}}{(2 \pi)^{3}} \\
& \times\left[\tilde{G}_{\mathbf{p}}^{22}\left(t_{2}, t_{3}\right) S_{\mathbf{k}^{\prime}}^{>}\left(t_{3}-t_{1}\right) \Delta_{\mathbf{q}^{\prime}}^{>}\left(t_{3}-t_{1}\right) \Delta_{\mathbf{q}}^{>}\left(t_{2}-t_{1}\right)\right. \\
& \left.-\tilde{G}_{\mathbf{p}}^{>}\left(t_{2}, t_{3}\right) S_{\mathbf{k}^{\prime}}^{11}\left(t_{3}-t_{1}\right) \Delta_{\mathbf{q}^{\prime}}^{11}\left(t_{3}-t_{1}\right) \Delta_{\mathbf{q}}^{>}\left(t_{2}-t_{1}\right)\right] P_{L} .
\end{aligned}
$$

Due to the chiral projections at the vertices, only the scalar parts of the nonequilibrium Majorana propagators contribute, which are the same for $\tilde{G}_{\mathbf{p}}^{>}, \tilde{G}_{\mathbf{p}}^{<}, \tilde{G}_{\mathbf{p}}^{11}$ and $\tilde{G}_{\mathbf{p}}^{22}$ (cf. Eqs. (A.19) - (A.24)

$$
P_{L} \bar{G}_{\mathbf{p}}\left(t, t^{\prime}\right) C P_{L}=\tilde{G}_{\mathbf{p}}\left(t, t^{\prime}\right) P_{L}, \quad \tilde{G}_{\mathbf{p}}\left(t, t^{\prime}\right)=\frac{M}{\omega_{\mathbf{p}}} \cos \left(\omega_{\mathbf{p}}\left(t-t^{\prime}\right)\right) f_{N}^{e q}\left(\omega_{\mathbf{p}}\right) e^{-\Gamma_{\mathbf{p}}\left(t+t^{\prime}\right) / 2} .
$$

The number of terms which contribute to the asymmetry $L_{\mathbf{k}}(t, t)$ can be significantly reduced by means of the following symmetry properties of the massless propagators:

$$
\begin{aligned}
S_{\mathbf{k}}^{>}(y)^{*} & =C S_{\mathbf{k}}^{<}(y) C^{-1}, & S_{\mathbf{k}}^{11}(y)^{*} & =C S_{\mathbf{k}}^{22}(y) C^{-1}, \\
\Delta_{\mathbf{q}}^{>}(y)^{*} & =\Delta_{\mathbf{q}}^{<}(y), & \Delta_{\mathbf{q}}^{11}(y)^{*} & =\Delta_{\mathbf{q}}^{22}(-y)
\end{aligned}
$$




$$
\begin{array}{ll}
S_{\mathbf{k}}^{<}(y)=\gamma_{5} S_{-\mathbf{k}}^{>}(-y) \gamma_{5}, & S_{\mathbf{k}}^{11}(y)=\gamma_{5} S_{-\mathbf{k}}^{11}(-y) \gamma_{5} \\
\Delta_{\mathbf{q}}^{<}(y)=\Delta_{\mathbf{q}}^{>}(-y), & \Delta_{\mathbf{q}}^{11}(y)=\Delta_{\mathbf{q}}^{11}(-y)
\end{array}
$$

Employing these transformation properties one can derive the following useful relations among different contributions to the integrand of Eq. (5.11):

$$
\begin{aligned}
& \operatorname{tr}\left[\Pi_{\mathbf{k}}^{(1,2)>}\left(t_{1}, t_{2}\right) S_{\mathbf{k}}^{<}\left(t_{2}-t_{1}\right)\right]=-\operatorname{tr}\left[\Pi_{\mathbf{k}}^{(1,2)<}\left(t_{1}, t_{2}\right) S_{\mathbf{k}}^{>}\left(t_{2}-t_{1}\right)\right]^{*}, \\
& \operatorname{tr}\left[\Pi_{\mathbf{k}}^{(1)>}\left(t_{1}, t_{2}\right) S_{\mathbf{k}}^{<}\left(t_{2}-t_{1}\right)\right]=-\operatorname{tr}\left[\Pi_{\mathbf{k}}^{(2)<}\left(t_{2}, t_{1}\right) S_{\mathbf{k}}^{>}\left(t_{1}-t_{2}\right)\right] .
\end{aligned}
$$

Using these relations one obtains from Eq. (5.11) the compact expression for the lepton asymmetry

$$
\begin{aligned}
L_{\mathbf{k} i i}(t, t)= & 12 \operatorname{Im}\left\{\lambda_{i 1}^{*}\left(\eta \lambda^{*}\right)_{i 1}\right\} \\
& \times \int_{0}^{t} d t_{1} \int_{0}^{t} d t_{2} \operatorname{Re}\left(\operatorname{tr}\left[\Pi_{\mathbf{k}}^{(2)>}\left(t_{1}, t_{2}\right) S_{\mathbf{k}}^{<}\left(t_{2}-t_{1}\right)\right]\right) .
\end{aligned}
$$

Since $\operatorname{Im}\left\{\lambda_{i 1}^{*}\left(\eta \lambda^{*}\right)_{j 1}\right\}=16 \pi \epsilon_{i j} /(3 M)$ (cf. Eq. (2.12)), the leading dependence of the flavourdiagonal lepton asymmetry $L_{\mathbf{k} i i}(t, t)$ on the Yukawa couplings is identical to the dependence of the difference $f_{\mathrm{L} i}(t, k)$ of lepton and anti-lepton distribution functions appearing in the Boltzmann equations.

To proceed further in the evaluation of $L_{\mathbf{k} i i}(t, t)$, the following relation can be used to simplify the integrand,

$$
\begin{aligned}
& S_{\mathbf{k}}^{22}(y) \Delta_{\mathbf{q}}^{22}(y)-S_{\mathbf{k}}^{<}(y) \Delta_{\mathbf{q}}^{<}(y)= \\
& \frac{\Theta(-y)}{2 q}\left[\gamma^{0}\left(\operatorname{coth}\left(\frac{\beta q}{2}\right) \cos (k y) \cos (q y)-\tanh \left(\frac{\beta k}{2}\right) \sin (k y) \sin (q y)\right)\right. \\
& \left.\quad-i \frac{M-\mathbf{k} \gamma}{k}\left(\tanh \left(\frac{\beta k}{2}\right) \cos (k y) \sin (q y)+\operatorname{coth}\left(\frac{\beta q}{2}\right) \sin (k y) \cos (q y)\right)\right] .
\end{aligned}
$$

One then obtains for the real part of the sum of products of thermal lepton and Higgs propagators $\left(y_{i j}=t_{i}-t_{j}\right)$,

$$
\begin{aligned}
& \operatorname{Re}\left(\operatorname{tr}\left[\left(S_{\mathbf{k}^{\prime}}^{22}\left(y_{31}\right) \Delta_{\mathbf{q}^{\prime}}^{22}\left(y_{31}\right)-S_{\mathbf{k}^{\prime}}^{<}\left(y_{31}\right) \Delta_{\mathbf{q}^{\prime}}^{<}\left(y_{31}\right)\right) S_{\mathbf{k}}^{<}\left(y_{21}\right)\right] \Delta_{\mathbf{q}}^{<}\left(y_{21}\right)\right)= \\
&-\frac{\Theta\left(y_{13}\right)}{16 q q^{\prime}}[\left(\operatorname{coth}\left(\frac{\beta q}{2}\right)\left(\cos \left((k+q) y_{21}\right)+\cos \left((k-q) y_{21}\right)\right)\right. \\
&\left.+\tanh \left(\frac{\beta k}{2}\right)\left(\cos \left((k+q) y_{21}\right)-\cos \left((k-q) y_{21}\right)\right)\right) \\
& \times\left(\operatorname{coth}\left(\frac{\beta q^{\prime}}{2}\right)\left(\cos \left(\left(k^{\prime}+q^{\prime}\right) y_{31}\right)+\cos \left(\left(k^{\prime}-q^{\prime}\right) y_{31}\right)\right)\right. \\
&\left.+\tanh \left(\frac{\beta k^{\prime}}{2}\right)\left(\cos \left(\left(k^{\prime}+q^{\prime}\right) y_{31}\right)-\cos \left(\left(k^{\prime}-q^{\prime}\right) y_{31}\right)\right)\right)
\end{aligned}
$$




$$
\begin{aligned}
+\frac{\mathbf{k} \cdot \mathbf{k}^{\prime}}{k k^{\prime}} & \left(\operatorname{coth}\left(\frac{\beta q}{2}\right)\left(\sin \left((k+q) y_{21}\right)+\sin \left((k-q) y_{21}\right)\right)\right. \\
& \left.+\tanh \left(\frac{\beta k}{2}\right)\left(\sin \left((k+q) y_{21}\right)-\sin \left((k-q) y_{21}\right)\right)\right) \\
\quad \times & \left(\operatorname{coth}\left(\frac{\beta q^{\prime}}{2}\right)\left(\sin \left(\left(k^{\prime}+q^{\prime}\right) y_{31}\right)+\sin \left(\left(k^{\prime}-q^{\prime}\right) y_{31}\right)\right)\right. \\
& \left.\left.+\tanh \left(\frac{\beta k^{\prime}}{2}\right)\left(\sin \left(\left(k^{\prime}+q^{\prime}\right) y_{31}\right)-\sin \left(\left(k^{\prime}-q^{\prime}\right) y_{31}\right)\right)\right)\right] .
\end{aligned}
$$

Defining the linear combinations of lepton and Higgs distribution functions (cf. (2.6) ),

$$
f_{l \phi}(k, q)=1-f_{l}(k)+f_{\phi}(q), \quad \bar{f}_{l \phi}(k, q)=f_{l}(k)+f_{\phi}(q),
$$

and using the relations

$$
\begin{aligned}
& \operatorname{coth}\left(\frac{\beta q}{2}\right)+\tanh \left(\frac{\beta k}{2}\right)=2 f_{l \phi}(k, q), \\
& \operatorname{coth}\left(\frac{\beta q}{2}\right)-\tanh \left(\frac{\beta k}{2}\right)=2 \bar{f}_{l \phi}(k, q),
\end{aligned}
$$

one finds

$$
\begin{aligned}
L_{\mathbf{k} i i}(t, t)=- & \epsilon_{i i} 32 \pi \int_{0}^{t} d t_{1} \int_{0}^{t} d t_{2} \int_{0}^{t_{2}} d t_{3} \int_{\mathbf{q}, \mathbf{q}^{\prime}} \frac{1}{\omega_{\mathbf{p}}} f_{N}^{e q}\left(\omega_{\mathbf{p}}\right) e^{-\frac{\Gamma}{2}\left(t_{1}+t_{3}\right)} \cos \left(\omega_{\mathbf{p}} y_{31}\right) \\
\times & {\left[\left(f_{l \phi}(k, q) \cos \left((k+q) y_{21}\right)+\bar{f}_{l \phi}(k, q) \cos \left((k-q) y_{21}\right)\right)\right.} \\
\times & \left(f_{l \phi}\left(k^{\prime}, q^{\prime}\right) \cos \left(\left(k^{\prime}+q^{\prime}\right) y_{23}\right)+\bar{f}_{l \phi}\left(k^{\prime}, q^{\prime}\right) \cos \left(\left(k^{\prime}-q^{\prime}\right) y_{23}\right)\right) \\
+\frac{\mathbf{k} \cdot \mathbf{k}^{\prime}}{k k^{\prime}} & \left(\left(f_{l \phi}(k, q) \sin \left((k+q) y_{21}\right)+\bar{f}_{l \phi}(k, q) \sin \left((k-q) y_{21}\right)\right)\right. \\
\times & \left.\left.\left(f_{l \phi}\left(k^{\prime}, q^{\prime}\right) \sin \left(\left(k^{\prime}+q^{\prime}\right) y_{23}\right)+\bar{f}_{l \phi}\left(k^{\prime}, q^{\prime}\right) \sin \left(\left(k^{\prime}-q^{\prime}\right) y_{23}\right)\right)\right)\right]
\end{aligned}
$$

where we have again used the notation

$$
\int_{\mathbf{q}} \cdots=\int \frac{d^{3} \mathbf{q}}{(2 \pi)^{3} 2 q} \cdots
$$

The functions $f_{l \phi}$ and $\bar{f}_{l \phi}$ are well known from Weldon's analysis of discontinuities in finite-temperature field theory [35]. The sum of statistical factors

$$
f_{l \phi}(k, q)=\left(1-f_{l}(k)\right)\left(1+f_{\phi}(q)\right)+f_{l}(k) f_{\phi}(q)
$$


corresponds to decays and inverse decays of the massive Majorana neutrinos whereas

$$
\bar{f}_{l \phi}(k, q)=f_{\phi}(q)\left(1-f_{l}(k)\right)+f_{l}(k)\left(1+f_{\phi}(q)\right)
$$

accounts for their disappearance or appearance where a single quant, lepton or Higgs, is absorbed from or emitted into the thermal bath. The function $f_{l \phi}$ contains the vacuum contribution, i.e., $f_{l \phi} \rightarrow 1$ as $\beta \rightarrow \infty$, whereas $\bar{f}_{l \phi} \rightarrow 0$.

We now have to perform the three time integrations in Eq. (5.38). It is convenient to express the products of cosine's and sine's as sum of products of exponentials. Each term then becomes a sum of four exponentials, where the energies $\omega, k \pm q$ and $k^{\prime} \pm q^{\prime}$ appear in different linear combinations, and the four complex conjugate exponentials. As an example, consider the integral

$$
\mathcal{I}(t)=\int_{0}^{t} d t_{1} \int_{0}^{t} d t_{2} \int_{0}^{t_{2}} d t_{3} e^{-i \Omega_{1} t_{1}+i \Omega_{2} t_{2}+i \Omega_{3} t_{3}} e^{-\frac{\Gamma}{2}\left(t_{1}+t_{3}\right)},
$$

with $\Omega_{1}=\omega_{\mathbf{p}}-k-q, \Omega_{3}=\omega_{\mathbf{p}}-q^{\prime}-k^{\prime}$, and $\Omega_{2}=\Omega_{1}-\Omega_{3}=k^{\prime}+q^{\prime}-k-q$. A straightforward calculation yields

$$
\mathcal{I}(t)+\mathcal{I}^{*}(t)=\frac{-\Gamma\left(e^{-\Gamma t}+\cos \left(\Omega_{2} t\right)-e^{-\frac{\Gamma t}{2}}\left(\cos \left(\Omega_{1} t\right)+\cos \left(\Omega_{3} t\right)\right)\right)+\mathcal{O}(t)}{\left(\Omega_{1}^{2}+\frac{\Gamma^{2}}{4}\right)\left(\Omega_{3}^{2}+\frac{\Gamma^{2}}{4}\right)},
$$

where

$$
\mathcal{O}(t)=\frac{2 \Omega_{1} \Omega_{3}+\frac{\Gamma^{2}}{2}}{\Omega_{2}}\left(\sin \left(\Omega_{2} t\right)-e^{-\frac{\Gamma t}{2}}\left(\sin \left(\Omega_{1} t\right)-\sin \left(\Omega_{3} t\right)\right)\right)
$$

is of higher order in $\Gamma$ at $\Omega_{1,3}=0$. Hence, this term does not contribute to the lepton asymmetry at leading order in $\Gamma$, i.e., in the Yukawa couplings.

The two contributions in Eq. (5.38), without and with the prefactor $\mathbf{k} \cdot \mathbf{k}^{\prime} /\left(k k^{\prime}\right)$, add up to a single term proportional to $k \cdot k^{\prime} /\left(k k^{\prime}\right)$ where $k \cdot k^{\prime}$ denotes the product of 4 -vectors. This is a consequence of Lorentz invariance of the vacuum contribution. The full result is now easily obtained from Eqs. (5.38) and (5.42) by adding the contributions with reversed sign of $q$ and/or $q^{\prime}$, accompanied by the corresponding substitution $f_{l \Phi} \rightarrow \bar{f}_{l \Phi}$. Omitting the subleading terms $\mathcal{O}$ (cf. (5.43) $)$, one finally obtains

$$
L_{\mathbf{k} i j}(t, t)=\sum_{a=1}^{4} L_{\mathbf{k} i j}^{a}(t, t),
$$

where

$$
L_{\mathbf{k} i i}^{a}(t, t)=-\epsilon_{i i} 8 \pi \int_{\mathbf{q}, \mathbf{q}^{\prime}} \frac{k \cdot k^{\prime}}{k k^{\prime} \omega_{\mathbf{p}}} f_{N}^{e q}\left(\omega_{\mathbf{p}}\right) \frac{1}{2} \Gamma \sum_{\alpha, \beta= \pm} \hat{L}_{\mathbf{k}, \mathbf{q}, \mathbf{q}^{\prime}}^{a}(t ; \alpha, \beta)
$$


and

$$
\begin{aligned}
& \hat{L}_{\mathbf{k}, \mathbf{q}, \mathbf{q}^{\prime}}^{1}(t ; \alpha, \beta)=\frac{f_{l \phi}(k, q) f_{l \phi}\left(k^{\prime}, q^{\prime}\right)}{\left(\left(\omega_{\mathbf{p}}-\alpha(k+q)\right)^{2}+\frac{\Gamma^{2}}{4}\right)\left(\left(\omega_{\mathbf{p}}-\beta\left(k^{\prime}+q^{\prime}\right)\right)^{2}+\frac{\Gamma^{2}}{4}\right)} \\
& \times\left(e^{-\Gamma t}+\cos \left[\left(\alpha(k+q)-\beta\left(k^{\prime}+q^{\prime}\right)\right) t\right]\right. \\
& \left.-e^{-\frac{\Gamma t}{2}}\left(\cos \left[\left(\omega_{\mathbf{p}}-\alpha(k+q)\right) t\right]+\cos \left[\left(\omega_{\mathbf{p}}-\beta\left(k^{\prime}+q^{\prime}\right)\right) t\right]\right)\right), \\
& \hat{L}_{\mathbf{k}, \mathbf{q}, \mathbf{q}^{\prime}}^{2}(t ; \alpha, \beta)=\frac{\bar{f}_{l \phi}(k, q) f_{l \phi}\left(k^{\prime}, q^{\prime}\right)}{\left(\left(\omega_{\mathbf{p}}-\alpha(k-q)\right)^{2}+\frac{\Gamma^{2}}{4}\right)\left(\left(\omega_{\mathbf{p}}-\beta\left(k^{\prime}+q^{\prime}\right)\right)^{2}+\frac{\Gamma^{2}}{4}\right)} \\
& \times\left(e^{-\Gamma t}+\cos \left[\left(\alpha(k-q)-\beta\left(k^{\prime}+q^{\prime}\right)\right) t\right]\right. \\
& \left.-e^{-\frac{\Gamma t}{2}}\left(\cos \left[\left(\omega_{\mathbf{p}}-\alpha(k-q)\right) t\right]+\cos \left[\left(\omega_{\mathbf{p}}-\beta\left(k^{\prime}+q^{\prime}\right)\right) t\right]\right)\right), \\
& \hat{L}_{\mathbf{k}, \mathbf{q}, \mathbf{q}^{\prime}}^{3}(t ; \alpha, \beta)=\frac{f_{l \phi}(k, q) \bar{f}_{l \phi}\left(k^{\prime}, q^{\prime}\right)}{\left(\left(\omega_{\mathbf{p}}-\alpha(k+q)\right)^{2}+\frac{\Gamma^{2}}{4}\right)\left(\left(\omega_{\mathbf{p}}-\beta\left(k^{\prime}-q^{\prime}\right)\right)^{2}+\frac{\Gamma^{2}}{4}\right)} \\
& \times\left(e^{-\Gamma t}+\cos \left[\left(\alpha(k+q)-\beta\left(k^{\prime}-q^{\prime}\right)\right) t\right]\right. \\
& \left.-e^{-\frac{\Gamma t}{2}}\left(\cos \left[\left(\omega_{\mathbf{p}}-\alpha(k+q)\right) t\right]+\cos \left[\left(\omega_{\mathbf{p}}-\beta\left(k^{\prime}-q^{\prime}\right)\right) t\right]\right)\right), \\
& \hat{L}_{\mathbf{k}, \mathbf{q}, \mathbf{q}^{\prime}}^{4}(t ; \alpha, \beta)=\frac{f_{l \phi}(k, q) f_{l \phi}\left(k^{\prime}, q^{\prime}\right)}{\left(\left(\omega_{\mathbf{p}}-\alpha(k-q)\right)^{2}+\frac{\Gamma^{2}}{4}\right)\left(\left(\omega_{\mathbf{p}}-\beta\left(k^{\prime}-q^{\prime}\right)\right)^{2}+\frac{\Gamma^{2}}{4}\right)} \\
& \times\left(e^{-\Gamma t}+\cos \left[\left(\alpha(k-q)-\beta\left(k^{\prime}-q^{\prime}\right)\right) t\right]\right. \\
& \left.-e^{-\frac{\Gamma t}{2}}\left(\cos \left[\left(\omega_{\mathbf{p}}-\alpha(k-q)\right) t\right]+\cos \left[\left(\omega_{\mathbf{p}}-\beta\left(k^{\prime}-q^{\prime}\right)\right) t\right]\right)\right) .
\end{aligned}
$$

This expression contains off-shell and memory effects which are not contained in Boltzmann equations. A detailed comparison will be given in the following section.

So far we have neglected the thermal damping widths of lepton and Higgs fields due to gauge interactions, which are known to be much larger than the width of the heavy Majorana neutrino, $\gamma_{l} \sim \gamma_{\Phi} \sim g^{2} T \gg \lambda^{2} M \sim \Gamma$, for $M \lesssim T$. To estimate their effect we replace the free equilibrium propagators by

$$
\Delta_{\mathbf{k}}^{\mathrm{eq} \pm}(y)=\Delta_{\mathbf{k}}^{ \pm}(y) e^{-\gamma_{\Phi}|y|}, \quad S_{\mathbf{k}}^{\mathrm{eq} \pm}(y)=S_{\mathbf{k}}^{ \pm}(y) e^{-\gamma_{l}|y|} .
$$

This has a drastic effect on the calculation described above. For the dominant term in Eq. (5.45),$\hat{L}_{\mathbf{k}, \mathbf{q}, \mathbf{q}^{\prime}}^{1}$ with $\alpha=\beta=1$, where the energy dominators can be $\mathcal{O}\left(\Gamma^{2}\right)$, one now finds $\left(\gamma=\gamma_{l}+\gamma_{\Phi}\right)$,

$$
\begin{array}{rl}
\bar{L}_{\mathbf{k} i i}(t, t)=-\epsilon_{i i} & 16 \pi \int_{\mathbf{q}, \mathbf{q}^{\prime}} \frac{k \cdot k^{\prime}}{k k^{\prime} \omega_{\mathbf{p}}} \\
& \times \frac{\gamma \gamma^{\prime}}{\left(\left(\omega_{\mathbf{p}}-k-q\right)^{2}+\gamma^{2}\right)\left(\left(\omega_{\mathbf{p}}-k^{\prime}-q^{\prime}\right)^{2}+\gamma^{\prime 2}\right)}
\end{array}
$$




$$
\begin{aligned}
& \times f_{l \phi}(k, q) f_{l \phi}\left(k^{\prime}, q^{\prime}\right) f_{N}^{e q}\left(\omega_{\mathbf{p}}\right) \\
& \times \frac{1}{\Gamma}\left(1-e^{-\Gamma t}\right)
\end{aligned}
$$

where $\gamma=\gamma(k, q)$ and $\gamma^{\prime}=\gamma^{\prime}\left(k^{\prime}, q^{\prime}\right)$. Note that now all memory effects have disappeared.

\section{Boltzmann vs Kadanoff-Baym}

Let us now consider in detail the relation between the two results obtained for the lepton asymmetry: Eq. (2.11) from the Boltzmann equations and Eqs. (5.44) - (5.49) and (5.51) from the Kadanoff-Baym equations.

Clearly, the overall CP asymmetry is identical in both cases and also the momentum integrations are very similar. Compared to the Boltzmann result the Kadanoff-Baym result has an additional statistical lepton-Higgs factor and expected off-shell energy denominators. Furthermore, there are 16 different terms corresponding to the various combinations of decay and inverse decay, appearance and dissappearance. The most striking difference is the time dependence of the integrand: the Boltzmann result has a simple exponential behaviour whereas the Kadanoff-Baym result has terms rapidly oscillating with time with frequencies $\mathcal{O}(M) \gg \Gamma$, a manifestation of memory effects.

The time-dependence is contained in the integral $\mathcal{I}(t)$ given in Eq. (5.41). Defining

$$
\bar{\Omega}_{1}=\Omega_{1}+\frac{i}{2} \Gamma, \quad \bar{\Omega}_{3}=\Omega_{3}+\frac{i}{2} \Gamma
$$

and using the identities $t_{3}=t_{1}+\left(t_{2}-t_{1}\right)+\left(t_{3}-t_{2}\right)$ and $\Omega_{2}=\Omega_{1}-\Omega_{3}$, one has (cf. (5.41) $)$,

$$
\mathcal{I}(t)=\int_{0}^{t} d t_{1} e^{-\Gamma t_{1}} \int_{-t_{1}}^{t-t_{1}} d t_{21} \int_{-t_{2}}^{0} d t_{32} e^{i \bar{\Omega}_{1} t_{21}+i \bar{\Omega}_{3} t_{32}}
$$

where $t_{i j}=t_{i}-t_{j}$. After performing the time-integrations, one obtains the result

$$
\mathcal{I}(t)=\frac{1}{i \bar{\Omega}_{3}}\left[\frac{1}{\left|\bar{\Omega}_{1}\right|^{2}}\left(e^{i \bar{\Omega}_{1} t}-1\right)\left(e^{-i \bar{\Omega}_{1}^{*} t}-1\right)-\frac{1}{\Omega_{2} \bar{\Omega}_{1}^{*}}\left(e^{i \Omega_{2} t}-1\right)\left(e^{-i \bar{\Omega}_{1}^{*} t}-1\right)\right],
$$

which satisfies

$$
\mathcal{I}(0)=\mathcal{I}^{\prime}(0)=\mathcal{I}^{\prime \prime}(0)=0, \quad \mathcal{I}^{\prime \prime \prime}(0) \neq 0 .
$$

For large times, $t \gg 1 / \Gamma$, there remains a term oscillating with time,

$$
\mathcal{I}(t) \sim \frac{1}{i \bar{\Omega}_{3}}\left[\frac{1}{\left|\bar{\Omega}_{1}\right|^{2}}+\frac{1}{\Omega_{2} \bar{\Omega}_{1}^{*}}\left(e^{i \Omega_{2} t}-1\right)\right] .
$$


This is in contrast to the Boltzmann result whose time-dependence is given by

$$
\mathcal{I}_{B}(t)=\frac{1-e^{-\Gamma t}}{\Gamma},
$$

with

$$
\mathcal{I}_{B}(0)=0, \quad \mathcal{I}_{B}^{\prime}(0) \neq 0,
$$

and $\mathcal{I}_{B}(t) \sim 1 / \Gamma=$ const for large times $t \gg 1 / \Gamma$.

Where is the Boltzmann result hidden in the Kadanoff-Baym result, and in which limit is it recovered? To answer this question it is instructive to consider a modified integral $\overline{\mathcal{I}}(t)$, where thermal damping rates $\gamma \sim \gamma^{\prime} \sim g^{2} T$ are included, which affect the dependence on the time differences $\left|t_{2}-t_{1}\right|$ and $\left|t_{3}-t_{2}\right|$ (cf. Fig. (7),

$$
\overline{\mathcal{I}}(t)=\int_{0}^{t} d t_{1} e^{-\Gamma t_{1}} \int_{-t_{1}}^{t-t_{1}} d t_{21} \int_{-t_{2}}^{0} d t_{32} e^{i \bar{\Omega}_{1} t_{21}-\gamma\left|t_{21}\right|} e^{i \bar{\Omega}_{3} t_{32}-\gamma^{\prime}\left|t_{32}\right|} .
$$

Compared to Eq. (5.41) the main difference is that the damping term in the $t_{21}$-integration changes sign at $t_{21}=0$. This is in contrast to the damping due to the Majorana neutrino decay width $\Gamma$.

Carrying out the time-integrations one now obtains the result

$$
\begin{aligned}
\overline{\mathcal{I}}(t)=\frac{1}{i \bar{\Omega}_{3}+\gamma^{\prime}} & {\left[\frac{1}{\left(i \bar{\Omega}_{1}-\gamma\right)\left(-i \bar{\Omega}_{1}^{*}+\gamma\right)} e^{\left(i \bar{\Omega}_{1}-\gamma\right) t}\left(e^{\left(-i \bar{\Omega}_{1}^{*}+\gamma\right) t}-1\right)\right.} \\
& -\frac{1}{\left(i \bar{\Omega}_{1}+\gamma\right)\left(-i \bar{\Omega}_{1}^{*}-\gamma\right)}\left(e^{\left(-i \bar{\Omega}_{1}^{*}-\gamma\right) t}-1\right) \\
& -\frac{1}{\left(i \Omega_{2}-\gamma-\gamma^{\prime}\right)\left(-i \bar{\Omega}_{1}^{*}+\gamma\right)} e^{\left(i \Omega_{2}-\gamma-\gamma^{\prime}\right) t}\left(e^{\left(-i \bar{\Omega}_{1}^{*}+\gamma\right) t}-1\right) \\
& +\frac{1}{\left(i \Omega_{2}+\gamma-\gamma^{\prime}\right)\left(-i \bar{\Omega}_{1}^{*}-\gamma\right)}\left(e^{\left(-i \bar{\Omega}_{1}^{*}-\gamma\right) t}-1\right) \\
& \left.+\frac{2 \gamma}{\bar{\Omega}_{1}^{2}+\gamma^{2}} \frac{1-e^{-\Gamma t}}{\Gamma}-\frac{2 \gamma}{\left(i \bar{\Omega}_{3}^{*}+\gamma^{\prime}\right)\left(\left(i \Omega_{2}-\gamma^{\prime}\right)^{2}-\gamma^{2}\right)}\left(e^{\left(-i \bar{\Omega}_{3}^{*}-\gamma^{\prime}\right) t}-1\right)\right] .
\end{aligned}
$$

The first four terms reduce to Eq. (6.3) for $\gamma=\gamma^{\prime}=0$. Particularly interesting is the last line in Eq. (6.9), which is a contribution from the point $t_{21}=t_{2}-t_{1}=0$, where the damping term changes sign. This local contribution contains the only term which is enhanced by $1 / \Gamma$ and has Boltzmann-like time-dependence,

$$
\overline{\mathcal{I}}(t) \supset \mathcal{I}_{B}(t)=\frac{2 \gamma}{\left(i \bar{\Omega}_{3}+\gamma^{\prime}\right)\left(\bar{\Omega}_{1}^{2}+\gamma^{2}\right)} \frac{1-e^{-\Gamma t}}{\Gamma} .
$$


Note that as consequence of thermal damping all oscillatory terms are exponentially suppressed for times $t>1 / \gamma$,

$$
\begin{aligned}
\overline{\mathcal{I}}(t) \sim \frac{1}{i \bar{\Omega}_{3}+\gamma^{\prime}} & {\left[\frac{2 \gamma}{\bar{\Omega}_{1}^{2}+\gamma^{2}} \frac{1-e^{-\Gamma t}}{\Gamma}+\frac{2 \gamma}{\left(i \bar{\Omega}_{3}^{*}+\gamma^{\prime}\right)\left(\left(i \Omega_{2}-\gamma^{\prime}\right)^{2}-\gamma^{2}\right)}\right.} \\
+ & \left.\frac{1}{\left(i \bar{\Omega}_{1}+\gamma\right)\left(-i \bar{\Omega}_{1}^{*}-\gamma\right)}-\frac{1}{\left(i \Omega_{2}+\gamma-\gamma^{\prime}\right)\left(-i \bar{\Omega}_{1}^{*}-\gamma\right)}\right] .
\end{aligned}
$$

The Boltzmann-like term (6.10), which originates from the point $t_{2}=t_{1}$, vanishes for $\gamma=0$.

What is the order of magnitude of the lepton asymmetry (5.44) relative to the Boltzmann result in the case $\gamma=\gamma^{\prime}=0$ ? The Kadanoff-Baym result depends on $\tau=\Gamma t$, like the Boltzmann result, and in addition on the dimensionless parameter $\Gamma / M \ll 1$. In appendix $\mathrm{C}$ we shown that

$$
\frac{L_{\mathbf{k}}(t, t)}{f_{L}(t, k)} \rightarrow 0, \quad \text { for } \quad \frac{\Gamma}{M} \rightarrow 0, \tau=\Gamma t \text { fixed }
$$

Hence, in this zero-width limit, due to rapid oscillations of the integrand, the lepton asymmetry obtained from the Kadanoff-Baym equation is at least $\mathcal{O}(\Gamma / M)$ relative to the Boltzmann lepton asymmetry.

We are thus led to the conclusion that the lepton asymmetry obtained from the Kadanoff-Baym equations does not contain the Boltzmann result as limiting case as long as free equilibrium propagators are used for lepton and Higgs fields. This may not be too surprising. After all, the underlying assumption in our calculation has been that (gauge) interactions, much faster than heavy neutrino decay, establish kinetic equilibrium for leptons and Higgs particles. These interactions will unavoidably lead to thermal damping widths much larger than $\Gamma$. If these interactions are not taken into account in the calculation of the lepton asymmetry, one misses the main contribution and obtains a misleading result. This means that at present the best estimate for the full quantum mechanical lepton asymmetry is given by Eq. (5.51), which leads to a temperature dependent suppression compared to the Boltzmann result.

Note that the proposed incorporation of thermal damping rates leads to a Boltzmannlike result, Eq. (5.51), which is valid for $t \gtrsim 1 / \Gamma$. For $t<1 / \Gamma$, all terms have to be kept, and one has $\left.\partial_{t} \bar{L}_{\mathbf{k}}(t, t)\right|_{t=0}=0$, which is a property of the exact result (5.8), contrary to the Boltzmann approximation.

\section{Numerical analysis}

Let us now quantitatively compare the Boltzmann result (2.14) for the lepton asymmetry

$$
f_{L i}(t, k)=f_{l i}(t, k)-f_{\overline{l i}}(t, k)
$$


with the Kadanoff-Baym result for the lepton asymmetry

$$
L_{\mathbf{k} i i}(t, t)=-\operatorname{tr}\left[\gamma_{0} S_{L \mathbf{k} i i}^{+}(t, t)\right] .
$$

For free fields in thermal equilibrium both expressions are identical. For the KadanoffBaym result we use Eq. (5.51) which includes the estimated effect of thermal widths for lepton and Higgs fields.

As shown in Appendix C, the Boltzmann result (2.14) can be reduced to a twodimensional momentum integral (cf. (C.15) ),

$$
f_{L i}(t, k)=-\frac{\epsilon_{i i}}{4 \pi} F_{\mathrm{B}}(k, \beta) \frac{1}{\Gamma}\left(1-e^{-\Gamma t}\right),
$$

where we have defined

$$
\begin{aligned}
F_{\mathrm{B}}(k, \beta)= & \frac{1}{k} \int_{p_{\min }(k)}^{\infty} d p \int_{k_{\min }^{\prime}(p)}^{k_{\max }^{\prime}(p)} k^{\prime} d k^{\prime} \frac{1}{\omega_{\mathbf{p}}} \\
& \times\left(1-\frac{2 \omega_{\mathbf{p}} k-M^{2}}{2 p k} \frac{2 \omega_{\mathbf{p}} k^{\prime}-M^{2}}{2 p k^{\prime}}\right) f_{l \phi}\left(k, \omega_{\mathbf{p}}-k\right) f_{N}^{e q}\left(\omega_{\mathbf{p}}\right)
\end{aligned}
$$

here $\omega_{\mathbf{p}}=\sqrt{M^{2}+\mathbf{p}^{2}}$, the bracket represents the product of 4 -vectors divided by the corresponding energies, $k \cdot k^{\prime} /\left(k k^{\prime}\right)$, and the integration boundaries are

$$
p_{\min }(k)=\frac{\left|M^{2}-4 k^{2}\right|}{4 k}, \quad k_{\min }^{\prime}=\frac{\omega_{\mathbf{p}}-p}{2}, \quad k_{\max }^{\prime}(p)=\frac{\omega_{\mathbf{p}}+p}{2}
$$

The dependence on temperature $(\beta=1 / T)$ enters through the equilibrium distribution functions of Higgs particles and leptons,

$$
\begin{aligned}
& f_{l \phi}(k, q)=1-f_{l}(k)+f_{\phi}(q), \quad q=\omega_{\mathbf{p}}-k \\
& f_{l}(k)=\frac{1}{e^{\beta k}+1}, \quad f_{\phi}(q)=\frac{1}{e^{\beta q}-1}, \quad f_{N}^{e q}\left(\omega_{\mathbf{p}}\right)=\frac{1}{e^{\beta \omega_{\mathbf{p}}}+1} .
\end{aligned}
$$

The Kadanoff-Baym result (5.51) for the lepton asymmetry, which includes effects of thermal damping, takes the same form as the Boltzmann result

$$
\bar{L}_{\mathbf{k} i i}(t, t)=-\frac{\epsilon_{i i}}{4 \pi} F_{\mathrm{KB}}(k, \beta) \frac{1}{\Gamma}\left(1-e^{-\Gamma t}\right) .
$$

Since the integrand of the momentum integrations contains two delta-functions less than the expression for the Boltzmann result, the function $F_{\mathrm{KB}}(k, \beta)$ can only be written as a four-dimensional integral (cf. (C.19) ),

$$
F_{\mathrm{KB}}(k, \beta)=\frac{1}{\pi^{2}} \frac{1}{k} \int_{p_{\min }(k)}^{\infty} d p \int_{k_{\min }^{\prime}(p)}^{k_{\max }^{\prime}(p)} k^{\prime} d k \int_{q_{-}}^{q_{+}} d q \int_{q_{-}^{\prime}}^{q_{+}^{\prime}} d q^{\prime} \frac{1}{\omega_{\mathbf{p}}}
$$




$$
\begin{aligned}
& \times\left(1-\frac{p^{2}+k^{2}-q^{2}}{2 p k} \frac{p^{2}+k^{\prime 2}-q^{\prime 2}}{2 p k^{\prime}}\right) f_{l \phi}(k, q) f_{l \phi}\left(k^{\prime}, q^{\prime}\right) f_{N}^{e q}\left(\omega_{\mathbf{p}}\right) \\
& \times \frac{\gamma \gamma^{\prime}}{\left(\left(\omega_{\mathbf{p}}-k-q\right)^{2}+\gamma^{2}\right)\left(\left(\omega_{\mathbf{p}}-k^{\prime}-q^{\prime}\right)^{2}+\gamma^{\prime 2}\right)}
\end{aligned}
$$

with the integration boundaries

$$
q_{ \pm}=|p \pm k|, \quad q_{ \pm}^{\prime}=\left|p \pm k^{\prime}\right|
$$

For the thermal widths we use the estimate $\gamma \simeq \gamma^{\prime} \sim \frac{6 g^{2}}{8 \pi} T \sim 0.1 T$ (cf. [44]). Note that the damping in a non-Abelian plasma is considerably stronger than in an electromagnetic plasma at the same temperature.

It is instructive to compare the Boltzmann and Kadanoff-Baym results with the prediction of quantum Boltzmann equations. As shown in [19, 20, these equations lead to an additional statistical factor compared to Boltzmann equations, which implies for the lepton asymmetry

$$
\begin{aligned}
F_{\mathrm{QB}}(k, \beta)= & \frac{1}{k} \int_{p_{\min }(k)}^{\infty} d p \int_{k_{\min }^{\prime}(p)}^{k_{\max }^{\prime}(p)} k^{\prime} d k^{\prime} \frac{1}{\omega_{\mathbf{p}}} \\
& \times\left(1-\frac{2 \omega_{\mathbf{p}} k-M^{2}}{2 p k} \frac{2 \omega_{\mathbf{p}} k^{\prime}-M^{2}}{2 p k^{\prime}}\right) f_{l \phi}\left(k, \omega_{\mathbf{p}}-k\right) f_{l \phi}\left(k^{\prime}, \omega_{\mathbf{p}}-k^{\prime}\right) f_{N}^{e q}\left(\omega_{\mathbf{p}}\right) .
\end{aligned}
$$

In [19,20], this enhancement has been included in an effective, temperature-dependent CP asymmetry.

In Fig. 8 Boltzmann and Kadanoff-Baym results for the lepton asymmetry are compared. At momenta $k \sim 0.2$, where both distributions peak, the differences are less than 20\%, at larger momenta they reach at most 50\% (cf. Fig. 10). At temperatures $T \sim 0.3$, where leptogenesis takes place for typical neutrino parameters [30, 45], differences are essentially negligible.

Boltzmann and quantum Boltzmann results for the lepton asymmetry are compared in Fig. 9. At momenta $k \sim 0.2$, where both distributions are maximal, the differences can exceed 100\%, and they remain large also at larger momenta (cf. Fig.10). An enhancement $\mathcal{O}(100 \%)$ at $T \sim 1$ is qualitatively consistent with the enhancement found for the temperature-dependent CP asymmetries in [19,20].

The Kadanoff-Baym result strongly depends on the size of the thermal damping rates. For $\gamma, \gamma^{\prime} \rightarrow 0$, off-shell effects dissappear, and the Kadanoff-Baym result approaches the quantum Boltzmann result. Numerically, already for $\gamma \simeq \gamma^{\prime} \sim 0.01 T$ the differences are negligible. However, in a non-Abelian plasma, damping rates are large and, as a consequence, they almost compensate the enhancement due to the additional statistical factor contained in the quantum Boltzmann as well as the Kadanoff-Baym result. We conclude that, according to our estimates, the conventional Boltzmann equations provide rather accurate predictions for the lepton asymmetry. 

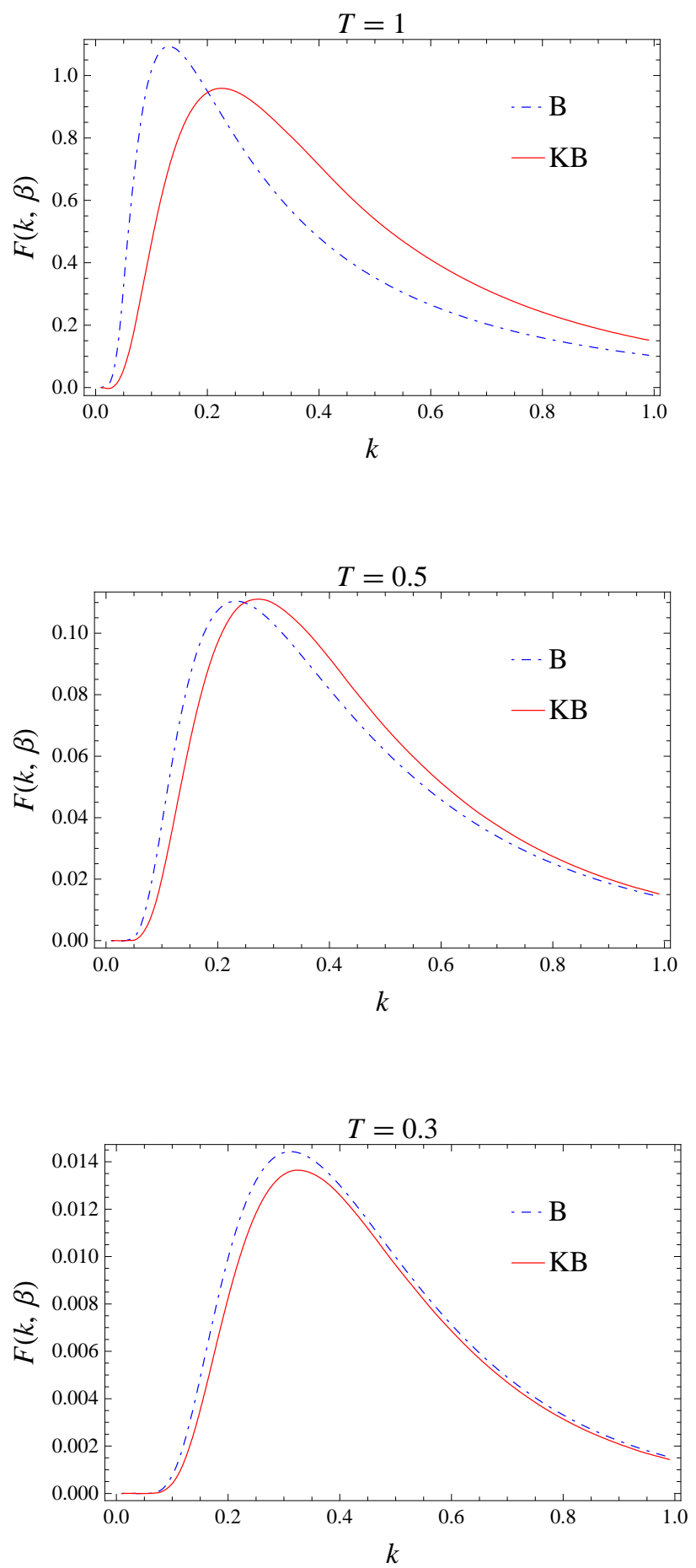

Figure 8: Comparison of the lepton asymmetry distribution functions obtained from Boltzmann equations (B, dot-dashed line) and Kadanoff-Baym equations (KB, full line) for three different temperatures; temperature and momentum are given in units of $M$. 

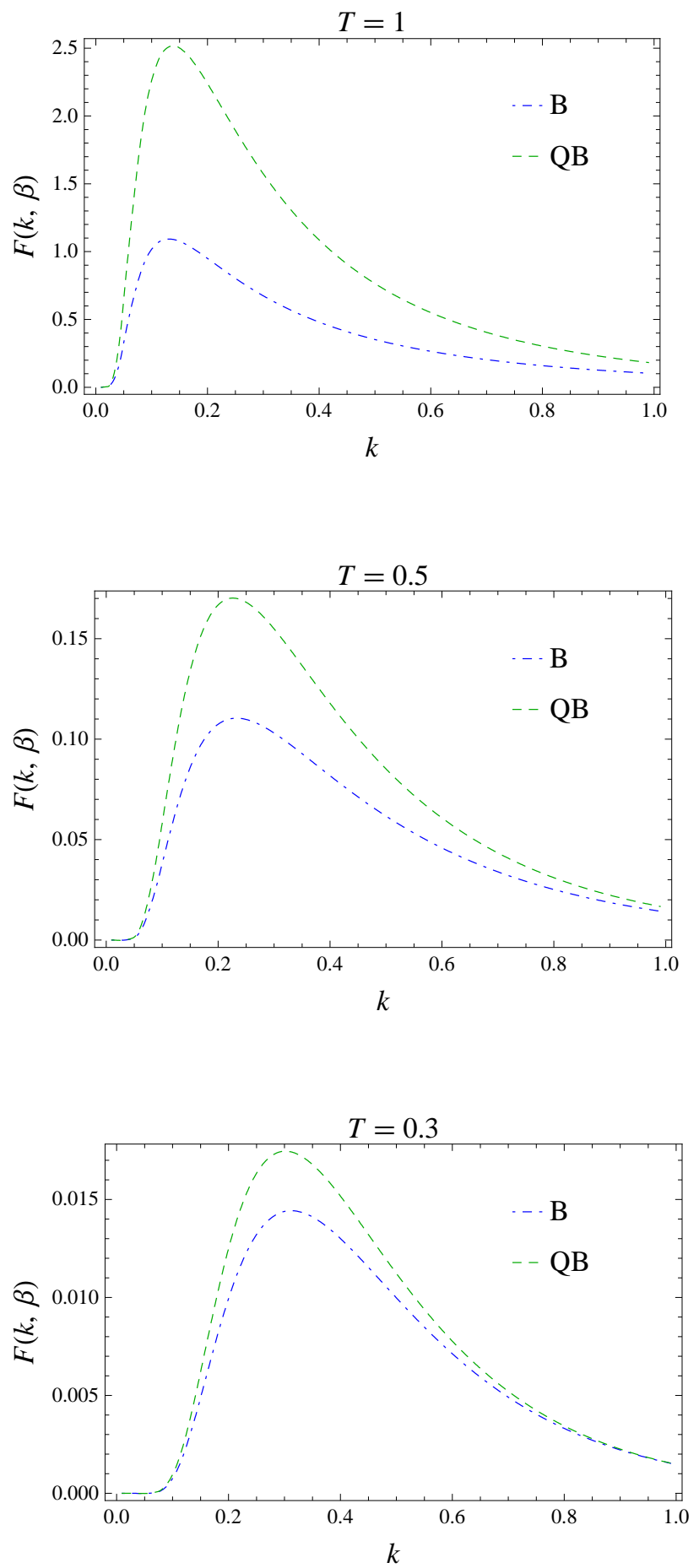

Figure 9: Comparison of the lepton asymmetry distribution functions obtained from Boltzmann equations ( $\mathrm{B}$, dot-dashed line) and quantum Boltzmann equations ( $\mathrm{QB}$, dashed line) for three different temperatures; temperature and momentum are given in units of $M$. 

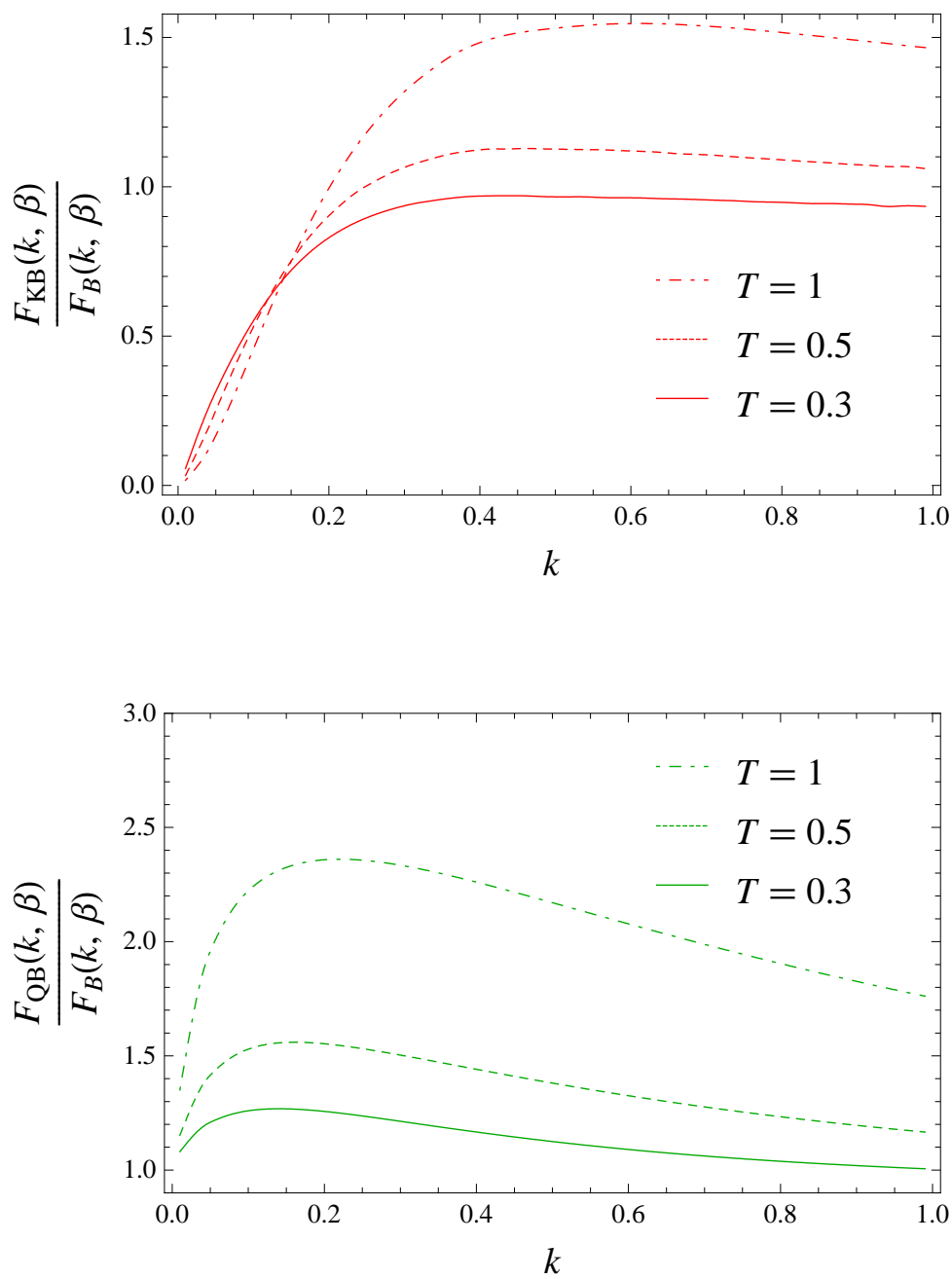

Figure 10: Ratio of Kadanoff-Baym and Boltzmann lepton asymmetries (upper panel) and ratio of quantum Boltzmann and Boltzmann lepton asymmetries (lower panel) for three different temperatures; temperature and momentum are given in units of $M$. 


\section{Summary and conclusions}

The goal of leptogenesis is the prediction of the cosmological baryon asymmetry, given neutrino masses an mixings. In a 'theory of leptogenesis', it must be possible to quantify the theoretical error on this prediction. This requires to go beyond Boltzmann as well as quantum Boltzmann equations, such that the size of memory and off-shell effects can be systematically computed.

In the present paper we have shown how to calculate the lepton asymmetry from first principles, i.e., in the framework of nonequilibrium quantum field theory. Our calculation is entirely based on Green's functions, and it therefore avoids all assumptions which are needed to arrive at Boltzmann equations.

Two key ingredients make the problem solvable. First, the thermal bath has a large number of degrees of freedom, all standard model particles, compared to only one particle out of equilibrium, the heavy neutrino. Hence, the backreaction of its equilibration on the temperature of the thermal bath can be neglected. Second, the heavy neutrino is only weakly coupled to the thermal bath and we can use perturbation theory in the corresponding Yukawa coupling $\lambda$.

The weak coupling of the heavy neutrino to the bath allowed us to obtain analytic expressions for the spectral function, which do not depend on initial conditions, and for the statistical propagator. In Section 4 we have discussed two solutions of the Kadanoff-Baym equations, which correspond to thermal and vacuum initial conditions. The statistical propagator which interpolates between vacuum at $t=0$ and thermal equilibrium at large times can then be used in the computation of the lepton asymmetry.

Thermal leptogenesis has two vastly different scales, the width $\Gamma$ of the heavy neutrino on one side, and its mass $M$, temperature $T$ of the bath and thermal damping widths $\gamma$ on the other side,

$$
\Gamma \sim \lambda^{2} M \ll \gamma \sim g^{2} T<T \lesssim M
$$

Typical leptogenesis parameters (cf. [2]) are $\Gamma \sim 10^{-7} M, \gamma \sim 0.1 T, T \sim 0.3 M, M \sim$ $10^{10} \mathrm{GeV}$. The existence of interactions in the plasma, which are fast compared to the equilibration time $\tau_{N}=1 / \Gamma$ of the heavy neutrino, is always implicitly assumed to justify the use of Boltzmann equations for the calculation of the asymmetry, but their effects are usually not explicitly taken into account.

The main result of this paper is the computation of the lepton asymmetry in Section 5 , where the nonequilibrium propagators of the heavy neutrino and free equilibrium propagators for massless lepton and Higgs fields are used. Compared to Boltzmann and quantum Boltzmann equations, the crucial difference of the result (5.44) - (5.49) are the memory effects, oscillations with frequencies $\mathcal{O}(M)$, much faster than the heavy neutrino equilibration time $\tau_{N}=1 / \Gamma$. These oscillations strongly suppress the generated lepton asymmetry $L_{\mathbf{k}}(t, t)$ compared to the Boltzmann result $f_{L}(t, k)$. In fact, as shown in appendix $\mathrm{C}$, the ratio $L_{\mathbf{k}}(t, t) / f_{L}(t, k)$ vanishes in the 'zero-width' limit $\Gamma / M \rightarrow 0$, with $\tau=\Gamma t$ fixed. 
This situation changes when the interactions, which in the Boltzmann approach are assumed to establish kinetic equilibrium, are explicitly included in the calculation. Lepton and Higgs fields in the thermal bath then acquire large thermal damping widths $\gamma \sim g^{2} T$, which cut off the oscillations. As a consequence, the predicted lepton asymmetry is similar to the quantum Boltzmann result, except for off-shell effects which are now included. For small damping widths, $\gamma \ll T$, the off-shell effects are negligible. They are large, however, in the standard model plasma. According to our calculation, using $\gamma \sim 0.1 T$, the damping effects essentially compensate the enhancement due to the additional statistical factor of the quantum Boltzmann equations. We conclude that, after all corrections are taken into account, the conventional Boltzmann equations again provide rather accurate predictions for the lepton asymmetry. Note that the classical Boltzmann behaviour emerges at large times, $t \gtrsim 1 / \Gamma>1 / \gamma$, while at early times all terms are of similar magnitude, and all quantum effects have to be kept.

As already emphasized in [8, it is of crucial importance to include gauge interactions in the Kadanoff-Baym approach to make further progress towards a 'theory of leptogenesis'. It remains to be seen whether the qualitative effects of thermal damping, as discussed in this paper, will then be confirmed or whether new surprises are encountered.

\section{Acknowlegements}

We would like to thank D. Bödeker, O. Philipsen, M. Shaposhnikov and C. Weniger for helpful discussions, and J. Schmidt for sharing his expertise. This work was supported by the German Science Foundation (DFG) within the Collaborative Research Center 676 "Particles, Strings and the Early Universe" and by the Swiss National Science Foundation. 


\section{A Thermal propagators}

In the following we list all propagators, which are needed in the calculation described in Section 5, as functions of relative time $y=t_{1}-t_{2}$ and total time $t=\left(t_{1}+t_{2}\right) / 2$.

- Free massive scalar $\left(\omega_{\mathbf{q}}=\sqrt{m^{2}+\mathbf{q}^{2}}\right)$

$$
\begin{aligned}
\Delta_{\mathbf{q}}^{-}(y) & =\frac{1}{\omega_{\mathbf{q}}} \sin \left(\omega_{\mathbf{q}} y\right) \\
\Delta_{\mathbf{q}}^{+}(y) & =\frac{1}{2 \omega_{\mathbf{q}}} \operatorname{coth}\left(\frac{\beta \omega_{\mathbf{q}}}{2}\right) \cos \left(\omega_{\mathbf{q}} y\right), \\
\Delta_{\mathbf{q}}^{11}(y) & =\frac{1}{2 \omega_{\mathbf{q}}}\left(\operatorname{coth}\left(\frac{\beta \omega_{\mathbf{q}}}{2}\right) \cos \left(\omega_{\mathbf{q}} y\right)-i \sin \left(\omega_{\mathbf{q}}|y|\right)\right) \\
& =\Delta_{\mathbf{q}}^{+}(y)-\frac{i}{2} \operatorname{sign}(y) \Delta_{\mathbf{q}}^{-}(y), \\
\Delta_{\mathbf{q}}^{22}(y) & =\frac{1}{2 \omega_{\mathbf{q}}}\left(\operatorname{coth}\left(\frac{\beta \omega_{\mathbf{q}}}{2}\right) \cos \left(\omega_{\mathbf{q}} y\right)+i \sin \left(\omega_{\mathbf{q}}|y|\right)\right) \\
& =\Delta_{\mathbf{q}}^{+}(y)+\frac{i}{2} \operatorname{sign}(y) \Delta_{\mathbf{q}}^{-}(y), \\
\Delta_{\mathbf{q}}^{>}(y) & =\frac{1}{2 \omega_{\mathbf{q}}}\left(\operatorname{coth}\left(\frac{\beta \omega_{\mathbf{q}}}{2}\right) \cos \left(\omega_{\mathbf{q}} y\right)-i \sin \left(\omega_{\mathbf{q}} y\right)\right), \\
\Delta_{\mathbf{q}}^{<}(y) & =\frac{1}{2 \omega_{\mathbf{q}}}\left(\operatorname{coth}\left(\frac{\beta \omega_{\mathbf{q}}}{2}\right) \cos \left(\omega_{\mathbf{q}} y\right)+i \sin \left(\omega_{\mathbf{q}} y\right)\right) .
\end{aligned}
$$

- Free massive Dirac fermion $\left(\omega_{\mathbf{k}}=\sqrt{m^{2}+\mathbf{k}^{2}}\right)$

$$
\begin{aligned}
S_{\mathbf{k}}^{-}(y)= & i \gamma_{0} \cos \left(\omega_{\mathbf{k}} y\right)+\frac{m-\mathbf{k} \boldsymbol{\gamma}}{\omega_{\mathbf{k}}} \sin \left(\omega_{\mathbf{k}} y\right) \\
S_{\mathbf{k}}^{+}(y)= & -\frac{1}{2} \tanh \left(\frac{\beta \omega_{\mathbf{k}}}{2}\right)\left(i \gamma_{0} \sin \left(\omega_{\mathbf{k}} y\right)-\frac{m-\mathbf{k} \boldsymbol{\gamma}}{\omega_{\mathbf{k}}} \cos \left(\omega_{\mathbf{k}} y\right)\right) \\
S_{\mathbf{k}}^{11}(y)= & \frac{\gamma_{0}}{2}\left(\cos \left(\omega_{\mathbf{k}} y\right) \operatorname{sign}(y)-i \tanh \left(\frac{\beta \omega_{\mathbf{k}}}{2}\right) \sin \left(\omega_{\mathbf{k}} y\right)\right) \\
& +\frac{m-\mathbf{k} \boldsymbol{\gamma}}{2 \omega_{\mathbf{k}}}\left(\tanh \left(\frac{\beta \omega_{\mathbf{k}}}{2}\right) \cos \left(\omega_{\mathbf{k}} y\right)-i \sin \left(\omega_{\mathbf{k}}|y|\right)\right) \\
= & S_{\mathbf{k}}^{+}(y)-\frac{i}{2} \operatorname{sign}(y) S_{\mathbf{k}}^{-}(y) \\
S_{\mathbf{k}}^{22}(y)= & \frac{\gamma_{0}}{2}\left(-\cos \left(\omega_{\mathbf{k}} y\right) \operatorname{sign}(y)-i \tanh \left(\frac{\beta \omega_{\mathbf{k}}}{2}\right) \sin \left(\omega_{\mathbf{k}} y\right)\right) \\
& +\frac{m-\mathbf{k} \boldsymbol{\gamma}}{2 \omega_{\mathbf{k}}}\left(\tanh \left(\frac{\beta \omega_{\mathbf{k}}}{2}\right) \cos \left(\omega_{\mathbf{k}} y\right)+i \sin \left(\omega_{\mathbf{k}}|y|\right)\right)
\end{aligned}
$$




$$
\begin{aligned}
& =S_{\mathbf{k}}^{+}(y)+\frac{i}{2} \operatorname{sign}(y) S_{\mathbf{k}}^{-}(y), \\
S_{\mathbf{k}}^{>}(y) & =\frac{\gamma_{0}}{2}\left(\cos \left(\omega_{\mathbf{k}} y\right)-i \tanh \left(\frac{\beta \omega_{\mathbf{k}}}{2}\right) \sin \left(\omega_{\mathbf{k}} y\right)\right) \\
& +\frac{m-\mathbf{k} \gamma}{2 \omega_{\mathbf{k}}}\left(\tanh \left(\frac{\beta \omega_{\mathbf{k}}}{2}\right) \cos \left(\omega_{\mathbf{k}} y\right)-i \sin \left(\omega_{\mathbf{k}} y\right)\right), \\
S_{\mathbf{k}}^{<}(y) & =\frac{\gamma_{0}}{2}\left(-\cos \left(\omega_{\mathbf{k}} y\right)-i \tanh \left(\frac{\beta \omega_{\mathbf{k}}}{2}\right) \sin \left(\omega_{\mathbf{k}} y\right)\right) \\
& +\frac{m-\mathbf{k} \gamma}{2 \omega_{\mathbf{k}}}\left(\tanh \left(\frac{\beta \omega_{\mathbf{k}}}{2}\right) \cos \left(\omega_{\mathbf{k}} y\right)+i \sin \left(\omega_{\mathbf{k}} y\right)\right) .
\end{aligned}
$$

The propagators for a massless left-handed fermion are obtained by the substitutions $\omega_{\mathbf{k}} \rightarrow k=|\mathbf{k}|, S_{\dddot{\mathbf{k}}} \rightarrow P_{L} S_{\mathfrak{\mathbf { k }}}$, where $P_{L}=\left(1-\gamma_{5}\right) / 2$.

- Free massive Majorana fermion $\left(\omega_{\mathbf{p}}=\sqrt{M^{2}+\mathbf{p}^{2}}\right)$

$$
\begin{aligned}
G_{\mathbf{p}}^{-}(y)= & \left(i \gamma_{0} \cos \left(\omega_{\mathbf{p}} y\right)+\frac{M-\mathbf{p} \gamma}{\omega_{\mathbf{p}}} \sin \left(\omega_{\mathbf{p}} y\right)\right) C^{-1} \\
G_{\mathbf{p}}^{+}(y)= & -\frac{1}{2} \tanh \left(\frac{\beta \omega_{\mathbf{p}}}{2}\right)\left(i \gamma_{0} \sin \left(\omega_{\mathbf{p}} y\right)-\frac{M-\mathbf{p} \boldsymbol{\gamma}}{\omega_{\mathbf{p}}} \cos \left(\omega_{\mathbf{p}} y\right)\right) C^{-1}, \\
G_{\mathbf{p}}^{11}(y)= & {\left[\frac{\gamma_{0}}{2}\left(\cos \left(\omega_{\mathbf{p}} y\right) \operatorname{sign}(y)-i \tanh \left(\frac{\beta \omega_{\mathbf{p}}}{2}\right) \sin \left(\omega_{\mathbf{p}} y\right)\right)\right.} \\
+ & \left.\frac{M-\mathbf{p} \boldsymbol{\gamma}}{2 \omega_{\mathbf{p}}}\left(\tanh \left(\frac{\beta \omega_{\mathbf{p}}}{2}\right) \cos \left(\omega_{\mathbf{p}} y\right)-i \sin \left(\omega_{\mathbf{p}}|y|\right)\right)\right] C^{-1} \\
G_{\mathbf{p}}^{22}(y)= & {\left[\frac{\gamma_{0}}{2}\left(-\cos \left(\omega_{\mathbf{p}} y\right) \operatorname{sign}(y)-i \tanh \left(\frac{\beta \omega_{\mathbf{p}}}{2}\right) \sin \left(\omega_{\mathbf{p}} y\right)\right)\right.} \\
+ & \left.\frac{M-\mathbf{p} \gamma}{2 \omega_{\mathbf{p}}}\left(\tanh \left(\frac{\beta \omega_{\mathbf{p}}}{2}\right) \cos \left(\omega_{\mathbf{p}} y\right)+i \sin \left(\omega_{\mathbf{p}}|y|\right)\right)\right] C^{-1} \\
G_{\mathbf{p}}^{>}(y)= & {\left[\frac{\gamma_{0}}{2}\left(\cos \left(\omega_{\mathbf{p}} y\right)-i \tanh \left(\frac{\beta \omega_{\mathbf{p}}}{2}\right) \sin \left(\omega_{\mathbf{p}} y\right)\right)\right.} \\
+ & \left.\frac{M-\mathbf{p} \boldsymbol{\gamma}}{2 \omega_{\mathbf{p}}}\left(\tanh \left(\frac{\beta \omega_{\mathbf{p}}}{2}\right) \cos \left(\omega_{\mathbf{p}} y\right)-i \sin \left(\omega_{\mathbf{p}} y\right)\right)\right] C^{-1} \\
& {\left[\frac{\gamma_{0}}{2}\left(-\cos \left(\omega_{\mathbf{p}} y\right)-i \tanh \left(\frac{\beta \omega_{\mathbf{p}}}{2}\right) \sin \left(\omega_{\mathbf{p}} y\right)\right)\right.} \\
G_{\mathbf{p}}^{<}(y) & \left.\frac{M-\mathbf{p} \boldsymbol{\gamma}}{2 \omega_{\mathbf{p}}}\left(\tanh \left(\frac{\beta \omega_{\mathbf{p}}}{2}\right) \cos \left(\omega_{\mathbf{p}} y\right)+i \sin \left(\omega_{\mathbf{p}} y\right)\right)\right] C^{-1}
\end{aligned}
$$


- Nonequilibrium massive Majorana fermion (interpolation between vacuum at $t=$ $y=0$ and thermal equilibrium at $t=\infty$, and memory integral)

$$
\begin{aligned}
G_{\mathbf{p}}^{-}(y)= & \left(i \gamma_{0} \cos \left(\omega_{\mathbf{p}} y\right)+\frac{M-\mathbf{p} \boldsymbol{\gamma}}{\omega_{\mathbf{p}}} \sin \left(\omega_{\mathbf{p}} y\right)\right) e^{-\Gamma_{\mathbf{p}}|y| / 2} C^{-1}, \\
G_{\mathbf{p}}^{+}(t, y)= & -\left(i \gamma_{0} \sin \left(\omega_{\mathbf{p}} y\right)-\frac{M-\mathbf{p} \boldsymbol{\gamma}}{\omega_{\mathbf{p}}} \cos \left(\omega_{\mathbf{p}} y\right)\right) \\
& \times\left(\frac{1}{2} \tanh \left(\frac{\beta \omega_{\mathbf{p}}}{2}\right) e^{-\Gamma_{\mathbf{p}}|y| / 2}+f_{N}^{e q}\left(\omega_{\mathbf{p}}\right) e^{-\Gamma_{\mathbf{p}} t}\right) C^{-1}, \\
G_{\mathbf{p}}^{11}(t, y)= & G_{\mathbf{p}}^{+}(t, y)-\frac{i}{2} \operatorname{sign}(y) G_{\mathbf{p}}^{-}(y), \\
G_{\mathbf{p}}^{22}(t, y)= & G_{\mathbf{p}}^{+}(t, y)+\frac{i}{2} \operatorname{sign}(y) G_{\mathbf{p}}^{-}(y), \\
G_{\mathbf{p}}^{>}(t, y)= & G_{\mathbf{p}}^{+}(t, y)-\frac{i}{2} G_{\mathbf{p}}^{-}(y), \\
G_{\mathbf{p}}^{<}(t, y)= & G_{\mathbf{p}}^{+}(t, y)+\frac{i}{2} G_{\mathbf{p}}^{+}(y), \\
G_{\mathbf{p}, \text { mem }}^{+}(t, y)= & -\frac{1}{2} \tanh \left(\frac{\beta \omega_{\mathbf{p}}}{2}\right)\left(i \gamma_{0} \sin \left(\omega_{\mathbf{p}} y\right)-\frac{M-\mathbf{p} \boldsymbol{c}}{\omega_{\mathbf{p}}} \cos \left(\omega_{\mathbf{p}} y\right)\right) \\
& \times\left(e^{-\Gamma_{\mathbf{p}}|y| / 2}-e^{-\Gamma_{\mathbf{p}} t}\right) C^{-1} .
\end{aligned}
$$

\section{B Feynman rules}

For completeness, we list in the following the Feynman rules for the Standard Model Lagrangian with right-handed neutrinos given in Eq. (1.2); $\alpha, \beta$ are spinor indices and $a, b, \ldots$ are $\mathrm{SU}(2)$ indices.

- Majorana neutrino

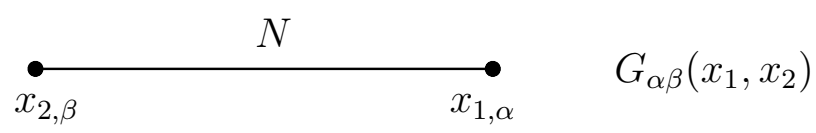

- Lepton doublet

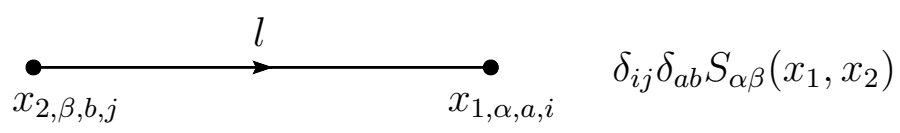


- Higgs doublet

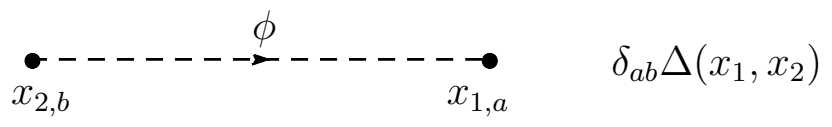

- Vertices
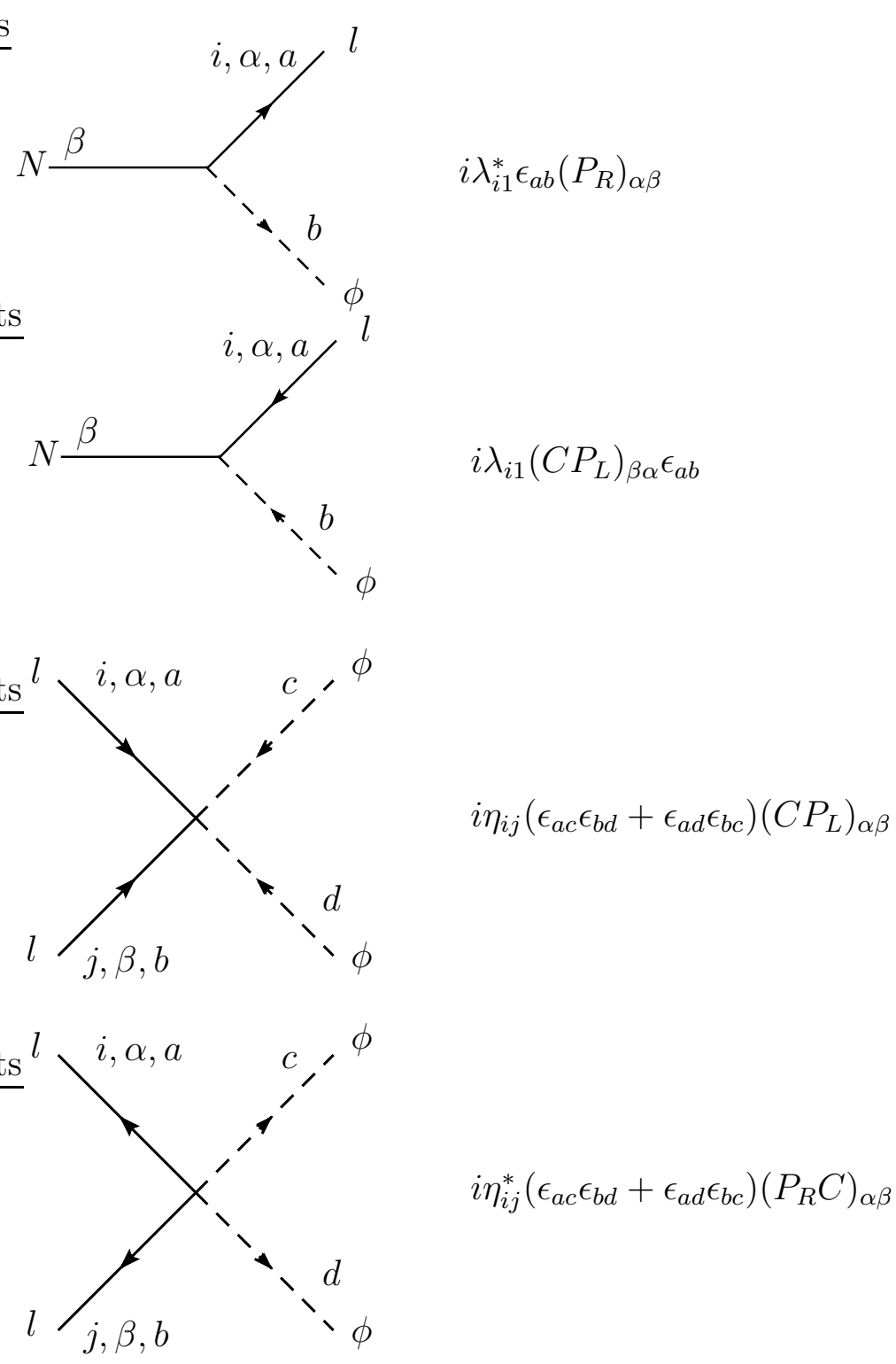


\section{Zero-width limit}

In this section we consider the Kadanoff-Baym result for the lepton asymmetry normalised to the Boltzmann result, $L_{\mathbf{k}}(t, t) / f_{L}(t, k)$, in the zero-width limit as defined in Eq. (6.12), i.e.,

$$
\frac{\Gamma}{M} \rightarrow 0, \quad \tau=\Gamma t \text { fixed } .
$$

To this end we have to evaluate the corresponding momentum integral (5.45) in this limit.

\section{C.1 Boltzmann equation}

Consider first the Boltzmann result for the lepton asymmetry given in Eq. (2.14),

$$
\begin{aligned}
f_{L i}(t, k)=-\epsilon_{i i} \frac{16 \pi}{k} & \int_{\mathbf{q}, \mathbf{p}, \mathbf{q}^{\prime}, \mathbf{k}^{\prime}} k \cdot k^{\prime}(2 \pi)^{4} \delta^{4}(k+q-p)(2 \pi)^{4} \delta^{4}\left(k^{\prime}+q^{\prime}-p\right) \\
& \times f_{l \phi}(k, q) f_{N}^{e q}\left(\omega_{\mathbf{p}}\right) \frac{1}{\Gamma}\left(1-e^{-\Gamma t}\right) .
\end{aligned}
$$

The integration over $\mathbf{q}$ and $\mathbf{q}^{\prime}$ can be performed using the $\delta$-functions, which leads to

$$
\begin{aligned}
f_{L i}(t, k)=-\frac{\epsilon_{i i}}{16 \pi^{3}} & \int d^{3} p \int d^{3} k^{\prime} \frac{k \cdot k^{\prime}}{k k^{\prime}} \frac{1}{\omega_{\mathbf{p}} q q^{\prime}} \delta\left(k+q-\omega_{\mathbf{p}}\right) \delta\left(k^{\prime}+q^{\prime}-\omega_{\mathbf{p}}\right) \\
& \times f_{l \phi}(k, q) f_{N}^{e q}\left(\omega_{\mathbf{p}}\right) \frac{1}{\Gamma}\left(1-e^{-\Gamma t}\right)
\end{aligned}
$$

where $q=|\mathbf{q}|$ and $q^{\prime}=\left|\mathbf{q}^{\prime}\right|$. The product of 4 -vectors, $k \cdot k^{\prime}=k k^{\prime}\left(1-\hat{\mathbf{k}} \cdot \hat{\mathbf{k}}^{\prime}\right)$, depends on the angles between the different momenta. It is convenient to define the angles with respect to the momentum p: $\theta=\angle(\mathbf{k}, \mathbf{p}), \theta^{\prime}=\angle\left(\mathbf{k}^{\prime}, \mathbf{p}\right)$ and $\varphi^{\prime}=\angle\left(\mathbf{k}_{\perp}, \mathbf{k}_{\perp}^{\prime}\right)$; here $\mathbf{k}_{\perp}$ and $\mathbf{k}_{\perp}^{\prime}$ are perpendicular to the vector $\mathbf{p}$, i.e., $\mathbf{k}=\mathbf{k}_{\|}+\mathbf{k}_{\perp}$ and $\mathbf{k}^{\prime}=\mathbf{k}_{\|}^{\prime}+\mathbf{k}_{\perp}^{\prime}$. In terms of these angles the unit vectors $\hat{\mathbf{k}}$ and $\hat{\mathbf{k}}^{\prime}$ are given by (see Fig. ???)

$$
\hat{\mathbf{k}}=\left(\begin{array}{c}
\cos \theta \\
\sin \theta \\
0
\end{array}\right), \quad \hat{\mathbf{k}}^{\prime}=\left(\begin{array}{c}
\cos \theta^{\prime} \\
\sin \theta^{\prime} \cos \varphi^{\prime} \\
\sin \theta^{\prime} \sin \varphi^{\prime}
\end{array}\right)
$$

with $\hat{\mathbf{k}} \cdot \hat{\mathbf{k}}^{\prime}=\cos \theta \cos \theta^{\prime}+\sin \theta \sin \theta^{\prime} \cos \varphi^{\prime}$. We then obtain

$$
\begin{aligned}
f_{L i}(t, \mathbf{k})=-\frac{\epsilon_{i i}}{16 \pi^{3}} & \int d^{3} p \int_{0}^{\infty} k^{\prime 2} d k^{\prime} \int_{-1}^{1} d \cos \theta^{\prime} \int_{0}^{2 \pi} d \varphi^{\prime} \frac{1}{\omega_{\mathbf{p}} q q^{\prime}} \\
& \times\left(1-\cos \theta \cos \theta^{\prime}-\sin \theta \sin \theta^{\prime} \cos \varphi^{\prime}\right) \\
& \times \delta\left(k+q-\omega_{\mathbf{p}}\right) \delta\left(k^{\prime}+q^{\prime}-\omega_{\mathbf{p}}\right) f_{l \phi}(k, q) f_{N}^{e q}\left(\omega_{\mathbf{p}}\right) \frac{1}{\Gamma}\left(1-e^{-\Gamma t}\right) .
\end{aligned}
$$




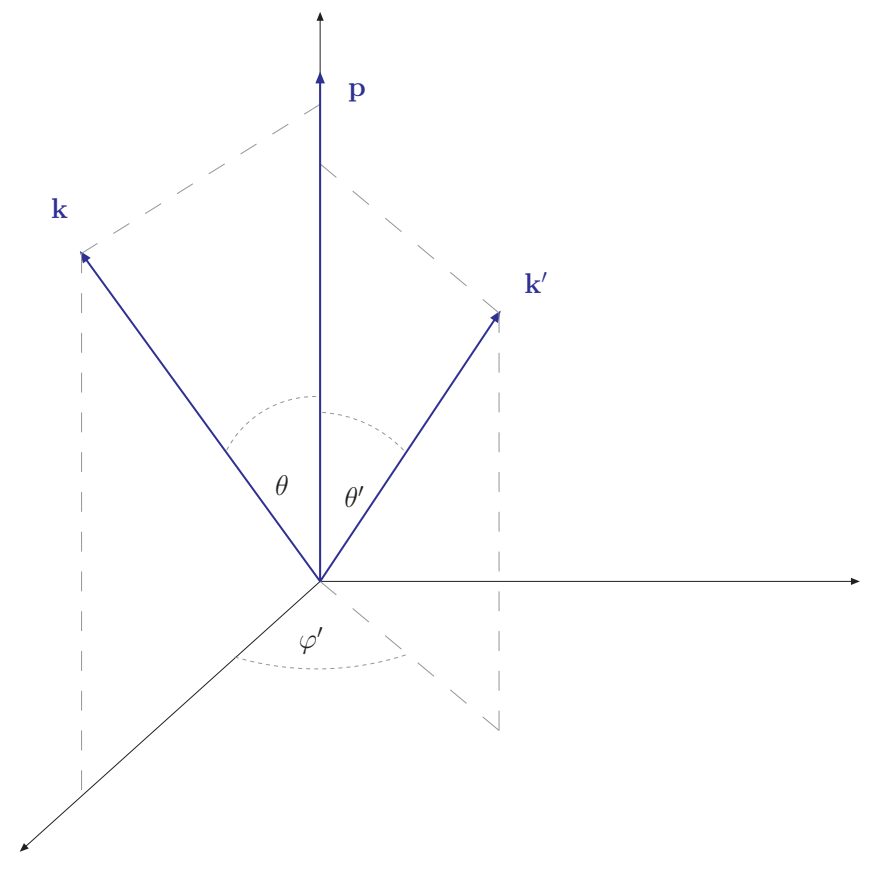

Figure 11: Integration angles

Momentum conservation relates the energies $q$ and $q^{\prime}$ to $p, k, k^{\prime}$ and the angles $\theta$ and $\theta^{\prime}$,

$$
\begin{aligned}
q & =|\mathbf{p}-\mathbf{k}|=\left(p^{2}+k^{2}-2 p k \cos \theta\right)^{1 / 2}, \\
q^{\prime} & =\left|\mathbf{p}-\mathbf{k}^{\prime}\right|=\left(p^{2}+k^{2}-2 p k \cos \theta^{\prime}\right)^{1 / 2} .
\end{aligned}
$$

We can now make use of rotational invariance of the distribution function,

$$
f_{L i}(t, k)=\frac{1}{4 \pi} \int d \Omega_{\mathbf{k}} f_{L i}(t, k)
$$

Changing variables,

$$
d q=-\frac{p k}{q} d \cos \theta, \quad d q^{\prime}=-\frac{p k^{\prime}}{q^{\prime}} d \cos \theta^{\prime},
$$

one arrives at

$$
\begin{aligned}
f_{L i}(t, k)=- & \frac{\epsilon_{i i}}{4 \pi} \frac{1}{k} \int d p \int_{0}^{\infty} k^{\prime} d k^{\prime} \int_{q_{+}}^{q_{-}} d q \int_{q_{+}^{\prime}}^{q_{-}^{\prime}} d q^{\prime}\left(1-\frac{p^{2}+k^{2}-q^{2}}{2 p k} \frac{p^{2}+k^{\prime 2}-q^{\prime 2}}{2 p k^{\prime}}\right) \\
& \left.\times \frac{1}{\omega_{\mathbf{p}}} \delta\left(k+q-\omega_{\mathbf{p}}\right) \delta\left(k^{\prime}+q^{\prime}-\omega_{\mathbf{p}}\right) f_{l \phi}(k, q) f_{N}^{e q}\left(\omega_{\mathbf{p}}\right) \frac{1}{\Gamma}\left(1-e^{-\Gamma t}\right), \quad \text { (C. } 9\right)
\end{aligned}
$$


where the limits of integration are given by the maximal and minimal value of $q$ and $q^{\prime}$, respectively,

$$
q_{ \pm}=|k \pm p|, \quad q_{ \pm}^{\prime}=\left|k^{\prime} \pm p\right|
$$

Consider now the argument of one $\delta$-function, $\Omega_{1}=\omega_{\mathbf{p}}-k-q$, with $\Omega_{1}^{\min }=\omega_{\mathbf{p}}-k-q_{+}$ and $\Omega_{1}^{\max }=\omega_{\mathbf{p}}-k-q_{-}$(cf. Eq.(5.41)). Obviously, the conditions $\Omega_{1}^{\min }<0$ and $\Omega_{1}^{\max }>0$ limit the integration range in $p$ for given momentum $k$,

$$
p>\frac{\left|M^{2}-4 k^{2}\right|}{4 k} \equiv p_{\min }(k) \text {. }
$$

Similarly, the constraint $p>\left(M^{2}-4 k^{\prime 2}\right) /\left(4 k^{\prime}\right)$ restricts the integration range in $k^{\prime}$ for given $p$,

$$
k^{\prime}>\frac{\omega_{\mathbf{p}}-p}{2} \equiv k_{\min }^{\prime}(p), \quad k^{\prime}<\frac{\omega_{\mathbf{p}}+p}{2} \equiv k_{\max }^{\prime}(p) .
$$

Changing again variables from $q$ and $q^{\prime}$ to $\Omega_{1}$ and $\Omega_{3}$, respectively, and using

$$
\frac{\partial\left(p, k^{\prime}, \Omega_{1}, \Omega_{3}\right)}{\partial\left(p, k^{\prime}, q, q^{\prime}\right)}=1
$$

the integral can now be written as

$$
\begin{aligned}
f_{L i}(t, k)=-\frac{\epsilon_{i i}}{4 \pi} \frac{1}{k} & \int_{p_{\min }(k)}^{\infty} d p \int_{k_{\min }^{\prime}(p)}^{k_{\max }^{\prime}(p)} d k^{\prime} \int_{\Omega_{1}^{\min }}^{\Omega_{1}^{\max }} d \Omega_{1} \int_{\Omega_{3}^{\min }}^{\Omega_{3}^{\max }} d \Omega_{3} \\
& \times \frac{1}{\omega_{\mathbf{p}}} \delta\left(\Omega_{1}\right) \delta\left(\Omega_{3}\right)\left(1-\frac{p^{2}+k^{2}-q^{2}}{2 p k} \frac{p^{2}+k^{\prime 2}-q^{\prime 2}}{2 p k^{\prime}}\right) \\
& \times f_{l \phi}(k, q) f_{N}^{e q}\left(\omega_{\mathbf{p}}\right) \frac{1}{\Gamma}\left(1-e^{-\Gamma t}\right) .
\end{aligned}
$$

The limits of integration have been chosen such that they contain the points $\Omega_{1}=0$ and $\Omega_{3}=0$, which correspond to energy conservation, $q=\omega_{\mathbf{p}}-k$ and $q^{\prime}=\omega_{\mathbf{p}}-k^{\prime}$, respectively. Hence, the integration on $\Omega_{1}$ and $\Omega_{3}$ can trivially be carried out, and we obtain the final result

$$
\begin{aligned}
f_{L i}(t, k)=-\frac{\epsilon_{i i}}{4 \pi} \frac{1}{k} & \int_{p_{\min }(k)}^{\infty} d p \int_{k_{\min }^{\prime}(p)}^{k_{\max }^{\prime}(p)} d k^{\prime} \frac{1}{\omega_{\mathbf{p}}}\left(1-\frac{2 \omega_{\mathbf{p}} k-M^{2}}{2 p k} \frac{2 \omega_{\mathbf{p}} k^{\prime}-M^{2}}{2 p k^{\prime}}\right) \\
& \times f_{l \phi}\left(k, \omega_{\mathbf{p}}-k\right) f_{N}^{e q}\left(\omega_{\mathbf{p}}\right) \frac{1}{\Gamma}\left(1-e^{-\Gamma t}\right) .
\end{aligned}
$$




\section{C.2 Kadanoff-Baym equation}

We are now ready to evaluate the leading contribution of the Kadanoff-Baym result for the lepton asymmetry. It is given by Eq. (5.46) with $\alpha=\beta=1$, and it can be written in the form

$$
\begin{array}{rl}
L_{\mathbf{k} i i}(t, t)=-\epsilon_{i i} & 8 \pi \int_{\mathbf{q}, \mathbf{q}^{\prime}} \frac{k \cdot k^{\prime}}{k k^{\prime} \omega_{\mathbf{p}}} f_{l \phi}(k, q) f_{l \phi}\left(k^{\prime}, q^{\prime}\right) f_{N}^{e q}\left(\omega_{\mathbf{p}}\right) \\
\times & \frac{\frac{1}{2} \Gamma}{\left(\left(\omega_{\mathbf{p}}-k-q\right)^{2}+\frac{\Gamma^{2}}{4}\right)\left(\left(\omega_{\mathbf{p}}-k^{\prime}-q^{\prime}\right)^{2}+\frac{\Gamma^{2}}{4}\right)} \\
\times & {\left[\left(e^{-\frac{\Gamma t}{2}}-\cos \left(\left(\omega_{\mathbf{p}}-k-q\right) t\right)\right)\left(e^{-\frac{\Gamma t}{2}}-\cos \left(\left(\omega_{\mathbf{p}}-k^{\prime}-q^{\prime}\right) t\right)\right)\right.} \\
& \left.-\sin \left(\left(\omega_{\mathbf{p}}-k-q\right) t\right) \sin \left(\left(\omega_{\mathbf{p}}-k^{\prime}-q^{\prime}\right) t\right)\right] .
\end{array}
$$

We first change variables, $\left(\mathbf{q}, \mathbf{q}^{\prime}\right) \rightarrow\left(\mathbf{p}, \mathbf{k}^{\prime}\right)$, with $\mathbf{p}=\mathbf{q}+\mathbf{k}=\mathbf{q}^{\prime}+\mathbf{k}^{\prime}$, and use rotational invariance,

$$
L_{\mathbf{k} i i}(t, t)=\frac{1}{4 \pi} \int d \Omega_{\mathbf{k}} L_{\mathbf{k} i i}(t, t) .
$$

Choosing again angles according to Fig. [??, the integral (C.16) becomes

$$
\begin{aligned}
L_{\mathbf{k} i i}(t, t) & \propto \int d \Omega_{\mathbf{k}} \int d \Omega_{\mathbf{k}^{\prime}} \int_{0}^{2 \pi} d \varphi^{\prime} \frac{k \cdot k^{\prime}}{k k^{\prime}} F\left(\theta, \theta^{\prime}, \cdots\right) \\
& =\int_{-1}^{1} d \cos \theta \int_{-1}^{1} d \cos \theta^{\prime} \int_{0}^{2 \pi} d \varphi^{\prime}\left(1-\cos \theta \cos \theta^{\prime}\right. \\
& \left.\quad-\sin \theta \sin \theta^{\prime} \cos \varphi^{\prime}\right) F\left(\theta, \theta^{\prime}, \cdots\right) \\
& =(2 \pi)^{2} \int_{-1}^{1} d \cos \theta \int_{-1}^{1} d \cos \theta^{\prime}\left(1-\cos \theta \cos \theta^{\prime}\right) F\left(\theta, \theta^{\prime}, \cdots\right),
\end{aligned}
$$

where we have used that the function $F\left(\theta, \theta^{\prime}, \ldots\right)$ does not depend on the angle $\varphi^{\prime}$. As in the previous section, we now change the integration variables from $\left(\theta, \theta^{\prime}\right)$ to $\left(q, q^{\prime}\right)$, and using Eq. (C.8) we obtain

$$
\begin{aligned}
L_{\mathbf{k} i i}(t, k)= & -\frac{\epsilon_{i i}}{8 \pi^{3}} \frac{1}{k} \int_{p_{\min }(k)}^{\infty} d p \int_{k_{\min }^{\prime}(p)}^{k_{\max }^{\prime}(p)} k^{\prime} d k^{\prime} \int_{q_{-}}^{q_{+}} d q \int_{q_{-}^{\prime}}^{q_{+}^{\prime}} d q^{\prime} \frac{1}{\omega_{\mathbf{p}}} \\
& \times\left(1-\frac{p^{2}+k^{2}-q^{2}}{2 p k} \frac{p^{2}+k^{2}-q^{\prime 2}}{2 p k^{\prime}}\right) f_{l \phi}(k, q) f_{l \phi}\left(k^{\prime}, q^{\prime}\right) f_{N}^{e q}\left(\omega_{\mathbf{p}}\right) \\
& \times \frac{\frac{1}{2} \Gamma}{\left(\left(\omega_{\mathbf{p}}-k-q\right)^{2}+\frac{\Gamma^{2}}{4}\right)\left(\left(\omega_{\mathbf{p}}-k^{\prime}-q^{\prime}\right)^{2}+\frac{\Gamma^{2}}{4}\right)}
\end{aligned}
$$




$$
\begin{aligned}
& \times\left[\left(e^{-\frac{\Gamma t}{2}}-\cos \left(\left(\omega_{\mathbf{p}}-k-q\right) t\right)\right)\left(e^{-\frac{\Gamma t}{2}}-\cos \left(\left(\omega_{\mathbf{p}}-k^{\prime}-q^{\prime}\right) t\right)\right)\right. \\
& \left.\quad-\sin \left(\left(\omega_{\mathbf{p}}-k-q\right) t\right) \sin \left(\left(\omega_{\mathbf{p}}-k^{\prime}-q^{\prime}\right) t\right)\right]
\end{aligned}
$$

where the limits of integration are given in Eqs. (C.10) - (C.12). We have restricted the integration over $p$ and $k$ to the range for which the intervals $\left[q_{-}, q_{+}\right]$and $\left[q_{-}^{\prime}, q_{+}^{\prime}\right]$ contain points satisfying $\omega_{\mathbf{p}}-k-q=0$ and $\omega_{\mathbf{p}}-k^{\prime}-q^{\prime}=0$, respectively. This finite part of the integral could then be $\mathcal{O}(1 / \Gamma)$, which is required to match the Boltzmann result for the lepton asymmetry. The remaining part is $\mathcal{O}(1)$ and therefore suppressed compared to the Boltzmann result.

Remarkably, the integral (C.19) is a sum of terms each of which factorizes into a product where one factor depends on $q$ but not on $q^{\prime}$, whereas the other factor depends on $q^{\prime}$ but not on $q$. Hence one obtains

$$
L_{\mathbf{k} i i}(t, t) \propto \int_{p_{\min }(k)}^{\infty} d p \int_{k_{\min }^{\prime}(p)}^{k_{\max }^{\prime}(p)} k^{\prime} d k^{\prime} \sum_{i} \mathcal{P}_{i}\left(q_{-}, q_{+}\right) \mathcal{Q}_{i}\left(q_{-}^{\prime}, q_{+}^{\prime}\right)
$$

where we have dropped the dependence of the factors $\mathcal{P}_{i}$ and $\mathcal{Q}_{i}$ on $k, p$ and $k^{\prime}$ for simplicity. Because of the factorization, we can now perform the integrations on $q$ and $q^{\prime}$ separately.

Naively, one may think that in the zero-width limit $\Gamma / M \rightarrow 0$ the cosine terms can be set to one. But for large time $t$, they oscillate fast, which leads to a different result. Consider the following contribution to the integral (C.19),

$$
\mathcal{P}\left(q_{-}, q_{+}\right)=-\int_{q_{-}}^{q_{+}} d q \frac{F(q)}{\left(\omega_{\mathbf{p}}-k-q\right)^{2}+\frac{\Gamma^{2}}{4}} \cos \left(\left(\omega_{\mathbf{p}}-k-q\right) t\right)
$$

where $F(q)$ has no poles. Changing the integration variable from $q$ to $z=2 \Omega_{1} / \Gamma$, with $\Omega_{1}=\omega_{\mathbf{p}}-k-q$, one obtains

$$
\begin{aligned}
\mathcal{P}\left(z_{\min }, z_{\max }\right)=\frac{i}{2 \Gamma} & \int_{z_{\min }}^{z_{\max }} d z F_{i}\left(\omega_{\mathbf{p}}-k-\frac{\Gamma}{2} z\right) \\
& \times\left(\frac{1}{z-i}-\frac{1}{z+1}\right)\left(e^{i z \frac{\Gamma}{2} t}+e^{-i z \frac{\Gamma}{2} t}\right)
\end{aligned}
$$

where $z_{\min }=2 \Omega_{1}^{\min } / \Gamma$ and $z_{\max }=2 \Omega_{1}^{\max } / \Gamma$, with $z_{\min }<0$ and $z_{\max }>0$. In the limit $\Gamma / M \rightarrow 0$ with $\tau=\Gamma t$ fixed, the integration limits approach $z_{\min } \rightarrow-\infty$ and $z_{\max } \rightarrow+\infty$, respectively. The integral is now easily evaluated by means of the residue theorem leading to the result

$$
\left.\Gamma \mathcal{P}\left(z_{\min }, z_{\max }\right)\right|_{\Gamma \rightarrow 0}=-\left.\pi\left(F\left(\omega_{\mathbf{p}}-k-i \frac{\Gamma}{2}\right) e^{-\frac{\tau}{2}}+F\left(\omega_{\mathbf{p}}-k+i \frac{\Gamma}{2}\right) e^{-\frac{\tau}{2}}\right)\right|_{\Gamma \rightarrow 0}
$$




$$
=-2 \pi F\left(\omega_{\mathbf{p}}-k\right) e^{-\frac{\tau}{2}}
$$

In Eq. (C.19) the term $\mathcal{P}$ appears together with a second term,

$$
\mathcal{P}^{\prime}\left(q_{-}, q_{+}\right)=\int_{q_{-}}^{q_{+}} d q \frac{F(q)}{\left(\omega_{\mathbf{p}}-k-q\right)^{2}+\frac{\Gamma^{2}}{4}} e^{-\frac{\tau}{2}},
$$

which can be evaluated in the same way as $\mathcal{P}$ in the zero-width limit, yielding

$$
\begin{aligned}
\left.\Gamma \mathcal{P}^{\prime}\left(z_{\min }, z_{\max }\right)\right|_{\Gamma \rightarrow 0} & =\left.\pi\left(F\left(\omega_{\mathbf{p}}-k-i \frac{\Gamma}{2}\right)+F\left(\omega_{\mathbf{p}}-k+i \frac{\Gamma}{2}\right)\right)\right|_{\Gamma \rightarrow 0} e^{-\frac{\tau}{2}} \\
& =2 \pi F\left(\omega_{\mathbf{p}}-k\right) e^{-\frac{\tau}{2}} .
\end{aligned}
$$

Clearly, the two terms $\mathcal{P}$ and $\mathcal{P}^{\prime}$ add up to zero. The same result is obtained for the second factor $\mathcal{Q}$ after the $q^{\prime}$ integration, as well as for the product of two sinus functions.

We conclude that the integral (C.16) does not contain a contribution $\mathcal{O}(1 / \Gamma)$. Hence, the ratio of Kadanoff-Baym result and Boltzmann result, $L_{\mathbf{k}}(t, t) / f_{L}(t, k)$, approaches zero in the limit $\Gamma / M \rightarrow 0, \tau=\Gamma t$ fixed.

\section{Equilibrium contribution}

In Section 5 we argued that the equilibrium part of the heavy neutrino propagator does not contribute to the lepton asymmetry. In this section we verify this claim.

The heavy neutrino propagator has an equilibrium and a nonequilibrium part,

$$
G_{\mathbf{p}}\left(t_{1}, t_{3}\right)=G_{\mathbf{p}}^{\mathrm{eq}}\left(t_{1}-t_{3}\right)+\tilde{G}_{\mathbf{p}}\left(t_{1}, t_{3}\right),
$$

whose main difference lies in the time dependence,

$$
G_{\mathbf{p}}^{\mathrm{eq}}\left(t_{1}-t_{3}\right) \propto e^{-\frac{\Gamma}{2}\left|t_{1}-t_{3}\right|}, \quad \tilde{G}_{\mathbf{p}}\left(t_{1}, t_{3}\right) \propto e^{-\frac{\Gamma}{2}\left(t_{1}+t_{3}\right)} .
$$

The computation of the lepton asymmetry in Section 5 was based on the nonequilbrium part, and it involved the time integral $\mathcal{I}$ (cf. Eq. (5.41)). Because of the different time dependence given in Eq. (D.2), the contribution of the equilibrium part to the asymmetry involves instead the integral

$$
\mathcal{J}(t)=\int_{0}^{t} d t_{1} \int_{0}^{t} d t_{2} \int_{0}^{t_{2}} d t_{3} e^{-i \Omega_{1} t_{1}+i \Omega_{2} t_{2}+i \Omega_{3} t_{3}} e^{-\frac{\Gamma}{2}\left|t_{1}-t_{3}\right|},
$$

which differs from $\mathcal{I}$ only with respect to the damping factor. $\Omega_{1}, \Omega_{2}$ and $\Omega_{3}$ are different linear combinations of energies, which satisfy $\Omega_{1}=\Omega_{2}+\Omega_{3}$.

In order to evaluate the integral $\mathcal{J}$, we have to split the time integration,

$$
\mathcal{J}(t)=\int_{0}^{t} d t_{1}\left[\int_{0}^{t_{1}} d t_{2} \int_{0}^{t_{2}} d t_{3} e^{-i \Omega_{1} t_{1}+i \Omega_{2} t_{2}+i \Omega_{3} t_{3}} e^{-\frac{\Gamma}{2}\left(t_{1}-t_{3}\right)}\right.
$$




$$
\begin{aligned}
+\int_{t_{1}}^{t} d t_{2} & \left(\int_{0}^{t_{1}} d t_{3} e^{-i \Omega_{1} t_{1}+i \Omega_{2} t_{2}+i \Omega_{3} t_{3}} e^{-\frac{\Gamma}{2}\left(t_{1}-t_{3}\right)}\right. \\
& \left.\left.+\int_{t_{1}}^{t_{2}} d t_{3} e^{-i \Omega_{1} t_{1}+i \Omega_{2} t_{2}+i \Omega_{3} t_{3}} e^{-\frac{\Gamma}{2}\left(t_{3}-t_{1}\right)}\right)\right] .
\end{aligned}
$$

Note the change of sign in the damping factor of the last two terms. As in Section 5, it is convenient to use the variables $\bar{\Omega}_{1}=\Omega_{1}-\frac{i}{2} \Gamma$ and $\bar{\Omega}_{3}=\Omega_{3}-\frac{i}{2} \Gamma$, for which the integral simplifies to

$$
\begin{aligned}
\mathcal{J}(t)=\int_{0}^{t} d t_{1} & {\left[\int_{0}^{t_{1}} d t_{2} \int_{0}^{t_{2}} d t_{3} e^{-i \bar{\Omega}_{1} t_{1}+i \Omega_{2} t_{2}+i \bar{\Omega}_{3} t_{3}}\right.} \\
+ & \left.\int_{t_{1}}^{t} d t_{2}\left(\int_{0}^{t_{1}} d t_{3} e^{-i \bar{\Omega}_{1} t_{1}+i \Omega_{2} t_{2}+i \bar{\Omega}_{3} t_{3}}+\int_{t_{1}}^{t_{2}} d t_{3} e^{-i \bar{\Omega}_{1}^{*} t_{1}+i \Omega_{2} t_{2}+i \bar{\Omega}_{3}^{*} t_{3}}\right)\right] .
\end{aligned}
$$

Performing the $t_{3}$ integral and using the relation $\Omega_{1}=\Omega_{2}+\Omega_{3}$, we obtain

$$
\begin{aligned}
\mathcal{J}(t)=\int_{0}^{t} d t_{1} & {\left[\int_{0}^{t_{1}} d t_{2} e^{-i \bar{\Omega}_{1} t_{1}} \frac{1}{i \bar{\Omega}_{3}}\left(e^{i \bar{\Omega}_{1} t_{2}}-e^{i \Omega_{2} t_{2}}\right)\right.} \\
& +\int_{t_{1}}^{t} d t_{2}\left(e^{-i \bar{\Omega}_{1} t_{1}} \frac{1}{i \bar{\Omega}_{3}}\left(e^{i \bar{\Omega}_{3} t_{1}}-1\right) e^{i \Omega_{2} t_{2}}\right. \\
& \left.\left.\quad+e^{-i \bar{\Omega}_{1}^{*} t_{1}} \frac{1}{i \bar{\Omega}_{3}^{*}}\left(e^{i \bar{\Omega}_{1}^{*} t_{2}}-e^{i \bar{\Omega}_{3}^{*} t_{1}} e^{i \Omega_{2} t_{2}}\right)\right)\right] .
\end{aligned}
$$

It is now straightforward to carry out the integrations over $t_{1}$ and $t_{2}$, which leads to

$$
\begin{aligned}
\mathcal{J}(t)+\mathcal{J}^{*}(t)= & \frac{2}{\left(\Omega_{1}^{2}+\frac{\Gamma^{2}}{4}\right)\left(\Omega_{3}^{2}+\frac{\Gamma^{2}}{4}\right)\left(\Omega_{1}-\Omega_{3}\right)} \times \\
& {\left[\Gamma\left(\Omega_{1}+\Omega_{3}\right)\left(\cos \left(\left(\Omega_{1}-\Omega_{3}\right) t\right)-1+\left(\cos \left(\Omega_{1} t\right)-\cos \left(\Omega_{3} t\right)\right) e^{-\frac{\Gamma t}{2}}\right)\right.} \\
& \left.+\left(2 \Omega_{1} \Omega_{3}-\frac{\Gamma^{2}}{2}\right)\left(\sin \left(\left(\Omega_{1}-\Omega_{3}\right) t\right)-\left(\sin \left(\Omega_{1} t\right)-\sin \left(\Omega_{3} t\right)\right) e^{-\frac{\Gamma t}{2}}\right)\right] .
\end{aligned}
$$

Note that the expression has no pole at $\Omega_{1}=\Omega_{3}$.

As in appendix $\mathrm{C}$ we now have to evaluate the momentum integral

$$
\mathcal{S}=\int_{\Omega_{1}^{\min }}^{\Omega_{1}^{\max }} d \Omega_{1} \int_{\Omega_{3}^{\min }}^{\Omega_{3}^{\max }} d \Omega_{3}\left(\mathcal{J}+\mathcal{J}^{*}\right),
$$

with the integration limits given below Eq. (C.10). To perform the zero-width limit, we again introduce the variables $z_{1,3}=2 \Omega_{1,3} / \Gamma$. For $\Gamma \rightarrow 0$, the limits of integration $z_{1,3}^{\min }$ and $z_{1,3}^{\max }$ approach $-\infty$ and $+\infty$, respectively. The $z_{3}$-integration can now be carried out by means of the residue theorem. The integrand of the remaining $z_{1}$-integration has a double pole. The integration can again be performed using the residue theorem, and we find that $\Gamma \mathcal{S}$ approaches zero in the limit $\Gamma / M \rightarrow 0, \tau=\Gamma t$ fixed. Hence, the equilibrium part of the heavy neutrino propagator does not contribute at leading order in $\Gamma / M$. 


\section{References}

[1] M. Fukugita and T. Yanagida, Phys. Lett. B 174 (1986) 45.

[2] For a review and references see, for example,

W. Buchmuller, R. D. Peccei and T. Yanagida, Ann. Rev. Nucl. Part. Sci. 55 (2005) 311 hep-ph/0502169];

S. Davidson, E. Nardi and Y. Nir, Phys. Rept. 466 (2008) 105 [0802.2962 [hep-ph]].

[3] V. A. Kuzmin, V. A. Rubakov and M. E. Shaposhnikov, Phys. Lett. B 155 (1985) 36.

[4] L. P. Kadanoff and G. Baym, Quantum Statistical Mechanics, Benjamin, New York, 1962

[5] J. Schwinger, J. Math. Phys. 2 (1961) 407.

[6] L. V. Keldysh, Zh. Eksp. Teor. Fiz. 47 (1964) 1515 [Sov. Phys. JETP 20 (1965) 1018].

[7] P. M. Bakschi and K. T. Mahanthappa, J. Math. Phys. 4 (1963) 1.

[8] A. Anisimov, W. Buchmuller, M. Drewes and S. Mendizabal, Phys. Rev. Lett. 104 (2010) 121102 [1001.3856 [hep-ph]].

[9] W. Buchmuller and S. Fredenhagen, Phys. Lett. B 483 (2000) 217 hep-ph/0004145.

[10] L. Covi, N. Rius, E. Roulet and F. Vissani, Phys. Rev. D 57 (1998) 93 [hep-ph/9704366.

[11] G. F. Giudice, A. Notari, M. Raidal, A. Riotto and A. Strumia, Nucl. Phys. B 685 (2004) 89 [hep-ph/0310123].

[12] C. P. Kiessig, M. Plumacher and M. H. Thoma, Phys. Rev. D 82 (2010) 036007 [1003.3016 [hep-ph]].

[13] A. Anisimov, D. Besak and D. Bodeker, 1012.3784 [hep-ph].

[14] M. Lindner and M. M. Muller, Phys. Rev. D 73 (2006) 125002 [hep-ph/0512147.

[15] M. Lindner and M. M. Muller, Phys. Rev. D 77 (2008) 025027 [0710.2917 [hep-ph]].

[16] A. De Simone and A. Riotto, JCAP 0708 (2007) 002 hep-ph/0703175].

[17] M. Garny, A. Hohenegger, A. Kartavtsev and M. Lindner, Phys. Rev. D 80 (2009) 125027 [0909.1559 [hep-ph]].

[18] M. Garny, A. Hohenegger, A. Kartavtsev and M. Lindner, Phys. Rev. D 81 (2010) 085027 [0911.4122 [hep-ph]]. 
[19] M. Beneke, B. Garbrecht, M. Herranen and P. Schwaller, Nucl. Phys. B 838 (2010) 1 [1002.1326 [hep-ph]].

[20] M. Garny, A. Hohenegger and A. Kartavtsev, Phys. Rev. D 81 (2010) 085028 [1002.0331 [hep-ph]].

[21] V. Cirigliano, C. Lee, M. J. Ramsey-Musolf and S. Tulin, Phys. Rev. D 81 (2010) 103503 [0912.3523 [hep-ph]].

[22] M. Beneke, B. Garbrecht, C. Fidler, M. Herranen and P. Schwaller, Nucl. Phys. B 843 (2011) 177 [1007.4783 [hep-ph]].

[23] B. Garbrecht, 1011.3122 [hep-ph].

[24] T. Prokopec, M. G. Schmidt and S. Weinstock, Annals Phys. 314 (2004) 208 hep-ph/0312110.

[25] T. Prokopec, M. G. Schmidt and S. Weinstock, Annals Phys. 314 (2004) 267 |hep-ph/0406140.

[26] T. Konstandin, T. Prokopec and M. G. Schmidt, Nucl. Phys. B 716 (2005) 373 hep-ph/0410135.

[27] T. Konstandin, T. Prokopec, M. G. Schmidt and M. Seco, Nucl. Phys. B 738 (2006) 1 hep-ph/0505103.

[28] M. Herranen, K. Kainulainen and P. M. Rahkila, 1006.1929 [hep-ph].

[29] A. Anisimov, W. Buchmuller, M. Drewes and S. Mendizabal, Annals Phys. 324 (2009) 1234 [0812.1934 [hep-th]].

[30] W. Buchmuller, P. Di Bari and M. Plumacher, Nucl. Phys. B 643 (2002) 367 [Erratumibid. B 793 (2008) 362] hep-ph/0205349.

[31] L. Covi, E. Roulet and F. Vissani, Phys. Lett. B 384 (1996) 169 hep-ph/9605319.

[32] M. Flanz, E. A. Paschos and U. Sarkar, Phys. Lett. B 345 (1995) 248 [Erratum-ibid. B 382 (1996) 447] |hep-ph/9411366].

[33] W. Buchmuller and M. Plumacher, Phys. Lett. B 431 (1998) 354 hep-ph/9710460.

[34] For a recent discussion and references see

F. Hahn-Woernle, M. Plumacher and Y. Y. Y. Wong, JCAP 0908 (2009) 028 [0907.0205 [hep-ph]].

[35] H. A. Weldon, Phys. Rev. D 28 (1983) 2007. 
[36] J. Berges, AIP Conf. Proc. 739 (2005) 3 [hep-ph/0409233].

[37] K. c. Chou, Z. b. Su, B. l. Hao and L. Yu, Phys. Rept. 118 (1985) 1.

[38] G. Raffelt, G. Sigl and L. Stodolsky, Phys. Rev. Lett. 70 (1993) 2363 [Erratum-ibid. 98 (2007) 069902] [hep-ph/9209276].

[39] G. Sigl and G. Raffelt, Nucl. Phys. B 406 (1993) 423.

[40] J. S. Gagnon, Nucl. Phys. A 820 (2009) 199C.

[41] J. S. Gagnon and M. Shaposhnikov, 1012.1126 [hep-ph].

[42] M. Garny and M. M. Muller, Phys. Rev. D 80 (2009) 085011 [0904.3600 [hep-ph]].

[43] M. Drewes, 1012.5380 [hep-th].

[44] M. Le Bellac, Thermal Field Theory, Cambridge University Press 1996

[45] W. Buchmuller, P. Di Bari and M. Plumacher, Annals Phys. 315 (2005) 305 |hep-ph/0401240|. 


\section{E Erratum}

1. Starting from Eq. (2.11), $f_{L i}$ has to be replaced by $f_{L i} / 2$; starting from Eq. (5.38), $L_{\mathbf{k} i i}$ has to be replaced by $L_{\mathbf{k} i i} / 2$. In Eq. (3.25) $P_{L}$ has to be replaced by $P_{L} \gamma^{0}$; in Eq. (5.33) $M$ has to be dropped; in the trace of Eq. (5.34) a factor $P_{L}$ has to be included; in Eqs. (5.19), (5.20) and (5.45) the sign on the r.h.s. has to be reversed. In Eqs. (C.14) and (C.15) $d k^{\prime}$ has to be replaced by $k^{\prime} d k^{\prime}$; in the first line of Eq. (C.18) the $\varphi^{\prime}$-integral has to be dropped and in the second line a factor $2 \pi$ has to be included.

2. The statement after Eq. (5.43), that $\mathcal{O}(t)$ does not contribute to the asymmetry to leading order in $\Gamma$, is not correct. The leading contribution is given by

$$
\begin{aligned}
L_{\mathbf{k} i i}^{\prime}(t, t)=-\epsilon_{i i} 16 \pi & \int_{\mathbf{q}, \mathbf{q}^{\prime}} \frac{k \cdot k^{\prime}}{k k^{\prime} \omega_{\mathbf{p}}} f_{N}^{e q}\left(\omega_{\mathbf{p}}\right) f_{l \phi}(k, q) f_{l \phi}\left(k^{\prime}, q^{\prime}\right) \frac{1}{\left(\Omega_{1}^{2}+\frac{\Gamma^{2}}{4}\right)\left(\Omega_{3}^{2}+\frac{\Gamma^{2}}{4}\right)} \\
& \times \frac{2 \Omega_{1} \Omega_{3}+\frac{\Gamma^{2}}{2}}{\Omega_{2}}\left(\sin \left(\Omega_{2} t\right)-e^{-\frac{\Gamma t}{2}}\left(\sin \left(\Omega_{1} t\right)-\sin \left(\Omega_{3} t\right)\right)\right) .
\end{aligned}
$$

Changing integration variables, as described in detail in Appendix C (cf. Eq. (C.19) and the following discussion), one obtains

$$
\begin{aligned}
L_{\mathbf{k} i i}^{\prime}(t, t)= & -\frac{\epsilon_{i i}}{2 \pi^{3} \Gamma} \frac{1}{k} \int_{p_{\min }(k)}^{\infty} d p \int_{k_{\min }^{\prime}(p)}^{k_{\max }^{\prime}(p)} k^{\prime} d k^{\prime} \int_{z_{1}^{\min }}^{z_{1}^{\max }} d z_{1} \int_{z_{3}^{\min }}^{z_{3}^{\max }} d z_{3} \frac{1}{\omega_{\mathbf{p}}} \\
& \times\left(1-\frac{p^{2}+k^{2}-q^{2}}{2 p k} \frac{p^{2}+k^{\prime 2}-q^{\prime 2}}{2 p k^{\prime}}\right) f_{l \phi}(k, q) f_{l \phi}\left(k^{\prime}, q^{\prime}\right) f_{N}^{e q}\left(\omega_{\mathbf{p}}\right) \\
& \times \frac{1}{\left(z_{1}^{2}+1\right)\left(z_{3}^{2}+1\right)} \frac{z_{1} z_{3}+1}{z_{1}-z_{3}}\left(\sin \left(\left(z_{1}-z_{3}\right) \frac{\tau}{2}\right)-e^{-\frac{\tau}{2}}\left(\sin \left(z_{1} \frac{\tau}{2}\right)-\sin \left(z_{3} \frac{\tau}{2}\right)\right)\right),
\end{aligned}
$$

where $\tau=\Gamma t, q=\omega_{\mathbf{p}}-k-\Gamma z_{1} / 2, q^{\prime}=\omega_{\mathbf{p}}-k^{\prime}-\Gamma z_{3} / 2$ and $z_{1,3}^{\min }=2 \Omega_{1,3}^{\min } / \Gamma, z_{1,3}^{\max }=2 \Omega_{1,3}^{\max } / \Gamma$, with $z_{1,3}^{\min }<0$ and $z_{1,3}^{\max }>0$. In the limit $\Gamma / M \rightarrow 0$ with $\tau=\Gamma t$ fixed, the integration limits approach $z_{1,3}^{\min } \rightarrow-\infty$ and $z_{1,3}^{\max } \rightarrow+\infty$, respectively. The integral can now be evaluated by means of Cauchy's theorem yielding the result

$$
\begin{aligned}
L_{\mathbf{k} i i}^{\prime}(t, t) \rightarrow & -\frac{\epsilon_{i i}}{2 \pi} \frac{1}{k} \int_{p_{\min }(k)}^{\infty} d p \int_{k_{\min }^{\prime}(p)}^{k_{\max }^{\prime}(p)} k^{\prime} d k^{\prime} \frac{1}{\omega_{\mathbf{p}}}\left(1-\frac{2 \omega_{\mathbf{p}} k-M^{2}}{2 p k} \frac{2 \omega_{\mathbf{p}} k^{\prime}-M^{2}}{2 p k^{\prime}}\right) \\
& \times f_{l \phi}\left(k, \omega_{\mathbf{p}}-k\right) f_{l \phi}\left(k, \omega_{\mathbf{p}}-k^{\prime}\right) f_{N}^{e q}\left(\omega_{\mathbf{p}}\right) \frac{1-e^{-\tau}}{\Gamma},
\end{aligned}
$$

which, up to the statistical factor, agrees with the Boltzmann result (C.15). Hence, contrary to statements in the paper, the Boltzmann result is also obtained for vanishing thermal damping widths in the limit $\Gamma \rightarrow 0$.

We thank Mathias Garny for pointing out to us that $\mathcal{O}(t)$ yields the Boltzmann result in the zero-width limit. 